LPT-07-25

CERN-PH-TH/2007-074

\title{
The MSSM with heavy scalars
}

\author{
Nicolás BERNAL ${ }^{1}$, AbDelhak DJOUADI $^{1}$ and Pietro SLAVICH ${ }^{2,3}$ \\ ${ }^{1}$ Laboratoire de Physique Théorique d'Orsay, UMR8627-CNRS, \\ Université Paris-Sud, Bât. 210, F-91405 Orsay Cedex, France. \\ ${ }^{2}$ LAPTH, 9 Chemin de Bellevue, F-74941 Annecy-le-Vieux, France. \\ ${ }^{3}$ CERN, Theory Division, CH-1211 Geneva 23, Switzerland.
}

\begin{abstract}
We perform a comprehensive analysis of the Minimal Supersymmetric Standard Model (MSSM) in the scenario where the scalar partners of the fermions and the Higgs particles (except for the Standard-Model-like one) are assumed to be very heavy and are removed from the low-energy spectrum. We first summarize our determination of the mass spectrum, in which we include the one-loop radiative corrections and resum to all orders the leading logarithms of the large scalar masses, and describe the implementation of these features in the FORTRAN code SuSpect which calculates the masses and couplings of the MSSM particles. We then study in detail the phenomenology of the model in scenarios where the gaugino mass parameters are non-universal at the GUT scale, which leads to very interesting features that are not present in the widely studied case of universal gaugino mass parameters. We discuss the constraints from collider searches and high-precision measurements, the cosmological constraints on the relic abundance of the neutralino candidate for the Dark Matter in the Universe - where new and interesting channels for neutralino annihilation appear - and the gluino lifetime. We then analyze, in the case of non-universal gaugino masses, the decays of the Higgs boson (in particular decays into and contributions of SUSY particles), of charginos and neutralinos (in particular decays into Higgs bosons and photons) and of gluinos, and highlight the differences from the case of universal gaugino masses.
\end{abstract}




\section{Introduction}

The main reason for introducing low energy Supersymmetry (SUSY) [1] in particle physics was its ability to solve the naturalness and hierarchy problems [2]. Indeed, SUSY prevents the Higgs boson mass from acquiring very large radiative corrections: the quadratically divergent loop contributions of the Standard Model (SM) particles to the squared Higgs masses are exactly canceled by the corresponding loop contributions of their supersymmetric partners. This cancellation stabilizes the huge hierarchy between the Grand Unification (GUT) and the electroweak symmetry breaking (EWSB) scales and no extreme fine tuning of parameters relevant to the mechanism of EWSB is required for this purpose. However, SUSY is not an exact symmetry and there is still a residual contribution to the Higgs masses that is proportional to the mass differences between the SM particles and the new SUSY particles. Therefore, for the fine-tuning problem not to be reintroduced in the theory, the mass $M_{S}$ of the new particles should be at most of the order of the TeV. The requirement of no fine tuning is the main reason for expecting SUSY particles to be accessible at the next generation of high-energy colliders, in particular at the Large Hadron Collider (LHC) and the International Linear Collider (ILC).

Nevertheless, there is no compelling criterion to define the maximal acceptable amount of fine tuning [3] and the choice of the upper bound on the SUSY scale $M_{S}$ is somewhat subjective. Thus, it might well be that $M_{S}$ is significantly larger than one $\mathrm{TeV}$, in which case the SUSY particles would be too heavy and not observable at the LHC. However, there are two more important motivations for SUSY that also call for some light SUSY particles: the consistent unification of the three gauge coupling constants at the GUT scale [4] and the solution to the Dark Matter (DM) problem [5]. Indeed, the SM slope of the evolution of the three gauge couplings has to be modified early enough by some SUSY particle contributions to achieve unification, and the DM problem calls for the existence of a new stable, neutral and weakly interacting particle that is not too heavy in order to have the required cosmological relic density. However, it has been pointed out [6] 8] that, for SUSY to provide solutions to the unification and DM problems, only gauginos and higgsinos, the spin- $\frac{1}{2}$ superpartners of the gauge and Higgs bosons, need to be relatively light, with masses of the order of the EWSB scale. The scalar partners of the SM fermions sit in complete irreducible representations of $\mathrm{SU}(5)$, therefore they could be very heavy without spoiling gauge coupling unification. For $M_{S} \gg 1 \mathrm{TeV}$ the model would be extremely fine-tuned, though, and one would have to give up SUSY as the solution to the hierarchy problem.

An interesting feature of such a scenario, commonly known as Split Supersymmetry [6,7], is that it is much more predictive than the general Minimal Supersymmetric Standard Model (MSSM) [9]. Indeed, it is well known that, in the most general case, the MSSM has a very large number of free parameters, $\mathcal{O}(100)$, most of them related to the sfermion sector. Even if one constrains the model to have a viable phenomenology, as in the socalled phenomenological MSSM [10] where minimal flavor mixing and CP violation as well 
as universality of the first- and second-generation sfermion masses are assumed, there are still more than 20 free parameters left. On the other hand, if the masses of all the scalars (except for one SM-like Higgs doublet) are pushed to large values only a handful of parameters are needed to describe the low-energy SUSY theory. As a by-product, such an assumption cures many problems of the general MSSM (such as the excess of flavor and CP violation, fast proton decay with dimension-5 operators, etc.) while still allowing for gauge coupling unification and a good DM candidate, the lightest of the neutralinos.

Indeed, in the MSSM with heavy scalars, besides a common value $M_{S}$ of the soft SUSYbreaking sfermion mass parameters, the basic inputs are essentially the three gaugino masses $M_{1}, M_{2}, M_{3}$, which can be unified to a common value at the high-energy GUT scale, the Higgs-higgsino mass parameter $\mu$ which is no longer fixed by the requirement of proper EWSB as in the general MSSM, and the parameter $\tan \beta$. The trilinear Higgs-sfermion couplings are forced to be small by the same symmetry that protects the higgsino and gaugino masses, and they play a very minor role. One can then derive in an exhaustive manner the relationship between this small number of inputs and the physical parameters, i.e. the (super)particle masses and couplings. However, if the scalars are very heavy, they will lead to significant quantum corrections in the Higgs and gaugino-higgsino sectors, enhanced by potentially large logarithms of the ratio between the EWSB scale and the scalar mass scale, $\log \left(M_{\mathrm{EWSB}} / M_{S}\right)$. In order to have reliable predictions, one has to properly decouple the heavy states from the low-energy theory and resum the large logarithmic corrections by means of Renormalization Group Equations (RGEs).

In fact, from a more practical point of view, in most (if not all) of the numerical RGE codes that calculate the Higgs and SUSY particle spectrum of the MSSM [11,12], one cannot assume too large masses for the sfermions, $M_{S} \gtrsim$ a few $\mathrm{TeV}$, as the programs become unreliable. Indeed, one has to perform major modifications to the general algorithms of such programs, which involve three main steps: the RG evolution, the consistent implementation of EWSB and the calculation of the mass spectra including the radiative corrections. In particular, one needs to properly decouple the heavy scalars and include the intermediate scale $M_{S}$ in the RG running of parameters back and forth between the low-energy scales $M_{Z}$ and $M_{\mathrm{EWSB}}$ and the high-energy scale $M_{\mathrm{GUT}}$; ignore the implementation of (radiative) EWSB as a large fine tuning is tolerated; resum the large logarithms in the radiative corrections to the calculated physical masses and couplings as mentioned above.

The purpose of this paper is to analyze, in the most comprehensive way, the SUSY and Higgs particle spectrum in the MSSM with heavy scalars, with $M_{S}$ ranging from a few TeV to $\sim 10^{12} \mathrm{GeV}$. We start by summarizing our determination of the particle spectrum of the low-energy theory in which the heavy scalars have been integrated out. We determine the masses and couplings of the Higgs boson and of the higgsinos and gauginos at the weak scale by solving the one-loop RGEs of Split SUSY [7, 13], thereby resumming to all orders the leading corrections that involve logarithms of the large scalar masses. We also include oneloop radiative corrections to the Higgs boson, chargino and neutralino masses, by adapting 
to the Split-SUSY case the MSSM formulae presented in ref. [14]; we investigate the variation of the masses with the renormalization scale, which can be considered as a rough estimate of the higher-order corrections. We also describe the implementation of these features in SuSpect [11, one of the public computer codes that calculate the MSSM mass spectrum.

We then study the regions in the parameter space that are compatible with the presently available collider data as well as with the constraints on the relic density of DM and on the gluino lifetime. In the former case we reemphasize that a new possibility [15], which is not present in the usual MSSM, has to be considered: the efficient annihilation of the DM lightest neutralinos through the exchange of the Higgs boson which decays into a real and a virtual $W$ boson, the latter subsequently decaying into two massless fermions. Finally, we have adapted to the Split-SUSY scenario the programs HDECAY [16] and SDECAY [17] for the calculation of the decay widths and branching ratios of the MSSM Higgs bosons and of the SUSY particles, respectively. We briefly discuss some features in the decays of the Higgs boson and the gauginos, focusing on interesting channels such as Higgs decays into invisible neutralinos and into two photons, chargino/neutralino decays into lighter ones and a Higgs boson and a photon, and gluino decays into a gluon and a neutralino.

Since the number of input parameters in the low-energy theory is rather limited, one can relax the usual assumption [18] of unified gaugino masses at the GUT scale, $M_{1}=M_{2}=$ $M_{3} \equiv m_{1 / 2}$, and still have a rather predictive model. Therefore, for the sake of generality, we will also consider specific patterns of non-universal gaugino masses [19] and, for illustration, we will discuss two different models: one in which SUSY-breaking occurs via an F-term that is not a $\mathrm{SU}(5)$ singlet [20] and another based on an orbifold string model [21]. In these models, the gaugino mass parameters at the electroweak scale can be widely different from the pattern of the universal scenario. In particular, the neutralino that is the lightest SUSY particle (LSP) can be, most of the time, either wino-like (as in anomaly mediated SUSYbreaking models for instance) or higgsino-like (which also occurs in the universal scenario for small values of the parameter $\mu$ ), leading to a near degeneracy of the LSP neutralino mass with the lightest chargino mass. In addition, in some of these scenarios, the LSP can also be close in mass with the gluino and/or a bino with a very small mass. This leads to several interesting phenomenological features that we will discuss in some detail, such as rapid annihilation of neutralino DM through the exchange of a $Z$ boson or co-annihilation of the gluinos which can be the next-to-lightest SUSY particle (NLSP).

The rest of the paper is organized as follows. In the next section we summarize the model with heavy scalars and discuss the implementation of the RGEs, the radiative corrections to the Higgs mass as well as to the gluino, chargino and neutralino masses, and the boundary conditions on the soft SUSY-breaking gaugino masses. In section 3 we summarize the various constraints on the model parameter space from collider data and cosmology. In section 4 we present some results on the Higgs boson decays involving SUSY particles and the decays of the chargino and neutralino states as well as the gluinos. Conclusions are given in section 5 . Finally, a set of useful formulae is collected in the appendix. 


\section{The low-energy effective theory}

In this section we summarize our determination of the mass spectrum of the effective theory valid below the scale $M_{S}$ at which all the heavy scalars of the MSSM, i.e. the sfermions and one Higgs doublet, are removed from the spectrum. We also discuss the different boundary conditions on the soft SUSY-breaking gaugino mass parameters that will be considered in the phenomenological analysis of sections 3 and 4 . Finally, a brief summary is given of how the model is implemented in the ForTRAn code SuSpect.

\subsection{Determination of the mass spectrum}

If the common mass of the scalars is assumed to be very large, $M_{S} \gg 1 \mathrm{TeV}$, the low-energy theory contains, besides the SM particles, the higgsinos $\tilde{H}_{u}, \tilde{H}_{d}$, the gluino $\tilde{g}$, the wino $\tilde{W}$ and the bino $\tilde{B}$. Omitting the gauge-invariant kinetic terms, as well as non-renormalizable operators suppressed by powers of the heavy scale $M_{S}$, the Lagrangian of the effective theory reads [7]

$$
\begin{aligned}
\mathcal{L} \supset & m^{2} H^{\dagger} H-\frac{\lambda}{2}\left(H^{\dagger} H\right)^{2}-\left[h_{i j}^{u} \bar{q}_{j} u_{i} \epsilon H^{*}+h_{i j}^{d} \bar{q}_{j} d_{i} H+h_{i j}^{e} \bar{\ell}_{j} e_{i} H\right. \\
& +\frac{1}{2} M_{3} \tilde{g}^{A} \tilde{g}^{A}+\frac{1}{2} M_{2} \tilde{W}^{a} \tilde{W}^{a}+\frac{1}{2} M_{1} \tilde{B} \tilde{B}+\mu \tilde{H}_{u}^{T} \epsilon \tilde{H}_{d} \\
& \left.+\frac{H^{\dagger}}{\sqrt{2}}\left(\tilde{g}_{u} \sigma^{a} \tilde{W}^{a}+\tilde{g}_{u}^{\prime} \tilde{B}\right) \tilde{H}_{u}+\frac{H^{T} \epsilon}{\sqrt{2}}\left(-\tilde{g}_{d} \sigma^{a} \tilde{W}^{a}+\tilde{g}_{d}^{\prime} \tilde{B}\right) \tilde{H}_{d}+\text { h.c. }\right],
\end{aligned}
$$

where $\sigma^{a}$ are the Pauli matrices, $\epsilon=i \sigma^{2}$ and $i, j$ are generation indices. The SM-like Higgs doublet $H$ is a linear combination of the two MSSM Higgs doublets $H_{u}$ and $H_{d}$, fine-tuned to have a small mass term $m^{2}$ :

$$
H=-\cos \beta \epsilon H_{d}^{*}+\sin \beta H_{u} .
$$

At the high scale $M_{S}$ the boundary conditions on the quartic Higgs coupling and on the Higgs-higgsino-gaugino couplings of the effective theory are determined by Supersymmetry:

$$
\begin{aligned}
\lambda\left(M_{S}\right) & =\frac{1}{4}\left[g^{2}\left(M_{S}\right)+g^{\prime 2}\left(M_{S}\right)\right] \cos ^{2} 2 \beta+\Delta_{\mathrm{th}} \lambda, \\
\tilde{g}_{u}\left(M_{S}\right) & =g\left(M_{S}\right) \sin \beta, \quad \tilde{g}_{d}\left(M_{S}\right)=g\left(M_{S}\right) \cos \beta \\
\tilde{g}_{u}^{\prime}\left(M_{S}\right) & =g^{\prime}\left(M_{S}\right) \sin \beta, \quad \tilde{g}_{d}^{\prime}\left(M_{S}\right)=g^{\prime}\left(M_{S}\right) \cos \beta .
\end{aligned}
$$

where $g$ and $g^{\prime}$ are the $\mathrm{SU}(2)$ and $\mathrm{U}(1)$ gauge couplings. Note that $\tan \beta$ is not a parameter of the low-energy effective theory, and it enters only the boundary conditions on the couplings. In fact, contrary to what happens in the usual MSSM, $\tan \beta$ is not interpreted here as the ratio of two Higgs vacuum expectation values, but rather as the fine-tuned angle that rotates the two Higgs doublets into one heavy and one light, SM-like doublet. 
In the boundary condition to the quartic Higgs coupling, we include also a one-loop threshold correction of $\mathcal{O}\left(h_{t}^{4}\right)$, where $h_{t}=m_{t} / v$ is the top quark Yukawa coupling,

$$
\Delta_{\mathrm{th}} \lambda=\frac{3 h_{t}^{4}}{8 \pi^{2}}\left[\left(1-\frac{g^{2}+g^{\prime 2}}{8 h_{t}^{2}}\right) \frac{X_{t}^{2}}{M_{S}^{2}}-\frac{X_{t}^{4}}{12 M_{S}^{4}}\right] .
$$

$X_{t}=A_{t}-\mu / \tan \beta$ is the left-right mixing of the stop squarks, with $A_{t}$ being the trilinear Higgs-stop coupling; here, $M_{S}$ is interpreted as the average of the two stop masses. Note, however, that in Split-SUSY models the trilinear coupling $A_{t}$ cannot be too large, since it is protected by the same symmetry that keeps the gaugino and the higgsino mass parameters small. The threshold correction in eq. (6) can thus be relevant only for relatively small values of $M_{S}$. Beyond tree level the boundary conditions in eqs. (3)-(5) are valid only in the $\overline{\mathrm{DR}}$ renormalization scheme. The one-loop electroweak corrections to eqs. (3)-(5) that account for the shift to the $\overline{\mathrm{MS}}$ scheme are given in the appendix.

The gauge and third-family Yukawa couplings as well as the vacuum expectation value (vev) of the SM Higgs field (normalized as $v \approx 174 \mathrm{GeV}$ ) are extracted from the following set of physical inputs [22,23]: the strong gauge coupling $\alpha_{s}\left(M_{Z}\right)=0.1187$; the electromagnetic coupling $\alpha\left(M_{Z}\right)=1 / 127.918$; the $Z$-boson mass $M_{Z}=91.1876 \mathrm{GeV}$; the Fermi constant $G_{F}=1.16637 \times 10^{-5} \mathrm{GeV}^{-2}$; the physical top and tau masses $M_{t}=170.9 \pm 1.8 \mathrm{GeV}$ and $M_{\tau}=1.777 \mathrm{GeV}$ and the running bottom mass $m_{b}\left(m_{b}\right)=4.25 \mathrm{GeV}$. We use one-loop formulae from ref. [14] to convert all the physical inputs into running parameters evaluated in the $\overline{\mathrm{MS}}$ scheme at a reference scale that we choose as equal to $M_{Z}$. To this purpose we need to adapt to the Split-SUSY scenario the formulae of ref. [14], which were originally derived for the MSSM in the $\overline{\mathrm{DR}}$ scheme: we remove the contributions of the heavy scalars and insert appropriate $\overline{\mathrm{DR}}-\overline{\mathrm{MS}}$ conversion factors. A summary of the relevant formulae is given in the appendix.

The gaugino masses are given as input at the GUT scale, defined as the scale where the two gauge couplings $g_{1} \equiv \sqrt{5 / 3} g^{\prime}$ and $g_{2} \equiv g$ unify, and evolved down to the scale $M_{S}$ by means of the one-loop RGEs of the MSSM. In addition to the minimal case where the three gaugino masses unify at the GUT scale, i.e. $M_{i}\left(M_{\mathrm{GUT}}\right)=m_{1 / 2}$, our analysis will consider scenarios of SUSY breaking in which the boundary conditions at the GUT scale are different. Finally, the $\mu$ parameter has to be provided as an independent input, contrary to the constrained MSSM case in which it can be extracted from the EWSB conditions, and we take it as a running parameter evaluated at the scale $M_{Z}$.

The parameters of the Lagrangian in eq. (1) are then evolved to a common renormalization scale $Q_{\mathrm{w}}$ of the order of the weak scale, by means of the one-loop RGEs of the Split-SUSY model, which we take from ref. [7]. Since some of the boundary conditions on the parameters are given at the SUSY scale $M_{S}$ and the others are given at the weak scale $M_{Z}$ an iterative procedure is necessary. The resulting couplings evaluated at the weak scale $Q_{\mathrm{w}}$ account for the all-order resummation of the leading logarithmic corrections involving powers of $\log \left(M_{S} / Q_{\mathrm{w}}\right)$. 
Once the iteration for the determination of the Lagrangian parameters converges, the physical masses of the Higgs boson, the charginos and the neutralinos are computed at the scale $Q_{\mathrm{w}}$ including one-loop radiative corrections; the gluino mass is computed separately at the scale $M_{3}$. The relation between the physical Higgs boson mass $M_{H}$ and the quartic coupling $\lambda$ computed at the generic scale $Q$ reads

$$
M_{H}=\sqrt{\frac{\lambda(Q)}{\sqrt{2} G_{F}}}\left[1+\delta^{\mathrm{SM}}(Q)+\delta^{\chi}(Q)\right] .
$$

The SM contribution $\delta^{\mathrm{SM}}$ can be found in ref. [24], and contains terms enhanced by $M_{t}^{4}$ coming from top-quark loops. The remaining term $\delta^{\chi}$ is the contribution of the diagrams involving charginos and neutralinos, and reads

$$
\delta^{\chi}=\frac{1}{2}\left[\frac{T_{H}^{\chi}}{\sqrt{2} m_{H}^{2} v}-\frac{\Pi_{H H}^{\chi}\left(m_{H}^{2}\right)}{m_{H}^{2}}+\frac{\Pi_{W W}^{\chi}(0)}{m_{W}^{2}}\right],
$$

where $m_{H}^{2}=2 \lambda v^{2}$ and $m_{W}^{2}=\frac{1}{2} g^{2} v^{2}$ are tree-level Higgs and $W$ masses, while $T_{H}^{\chi}, \Pi_{H H}^{\chi}$ and $\Pi_{W W}^{\chi}$ denote the chargino and neutralino contributions to the Higgs boson tadpole, the Higgs boson self-energy and the $W$ boson self-energy at zero external momentum, respectively. The explicit dependence of tadpole and self-energies on the renormalization scale compensates, up to higher-order (i.e. two-loop) effects, the implicit scale dependence of the Higgs quartic coupling in eq. (7). We extracted the formulae for the Higgs tadpole and the self-energies from the MSSM results of ref. [14], by appropriately rotating the Higgs fields, dropping the contributions of heavy scalars and expressing the couplings of charginos and neutralinos in terms of the effective Higgs-higgsino-gaugino couplings $\tilde{g}_{u, d}$ and $\tilde{g}_{u, d}^{\prime}$. We have also checked that our results for $\delta^{\chi}$ agree with the recent computation of ref. [25]. The relevant formulae can be found in the appendix.

The logarithmic dependence of $M_{H}$ on the sfermion mass scale $M_{S}$ has been discussed in earlier papers [6, 7, 13, 25, but we show it for completeness in fig. 1, choosing the SUSY input parameters as $A_{t}=0, \mu\left(M_{Z}\right)=m_{1 / 2}=500 \mathrm{GeV}$ and $\tan \beta=2$ or 30 . The top Yukawa coupling entering the one-loop corrections to the Higgs mass, eqs. (6) and (17), is expressed in terms of the running top mass, which is in turn extracted from the physical top mass via suitable threshold corrections. For each choice of $\tan \beta$ the solid lines in fig. 1 are obtained with the central value of the physical top mass, $M_{t}=170.9 \mathrm{GeV}$, and the dashed lines correspond to a $\pm 1 \sigma$ variation in the top mass. It can be seen that, for relatively low values of $M_{S}$, going from small to large $\tan \beta$ can change the Higgs boson mass by nearly 20 $\mathrm{GeV}$. This is the most important way in which the parameter $\tan \beta$ affects the low-energy phenomenology of the model, and it is due to the $\cos ^{2} 2 \beta$ term in the tree-level part of the boundary condition on the Higgs quartic coupling $\lambda$, eq. (3). When $M_{S}$ gets larger, however, the low-energy value of $\lambda$ is dominated by the radiative correction induced by the $\mathrm{RG}$ evolution, and the effect of the variation in $\tan \beta$ becomes less important. 


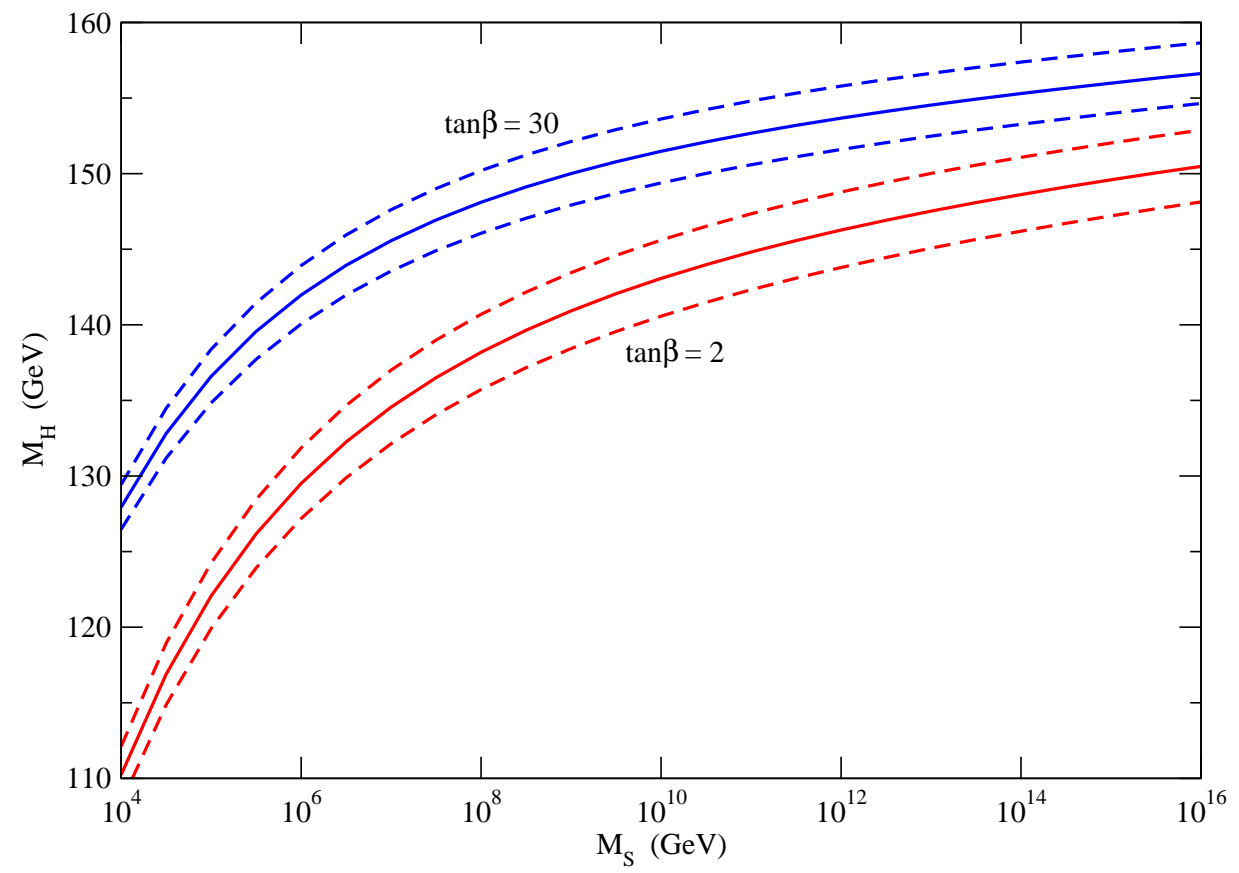

Figure 1: The Higgs boson mass as a function of the scalar mass $M_{S}$ for $A_{t}=0, \mu=m_{1 / 2}=$ $500 \mathrm{GeV}$ and two values of $\tan \beta$. The dashed lines correspond to a $\pm 1 \sigma$ variation of the top quark mass.

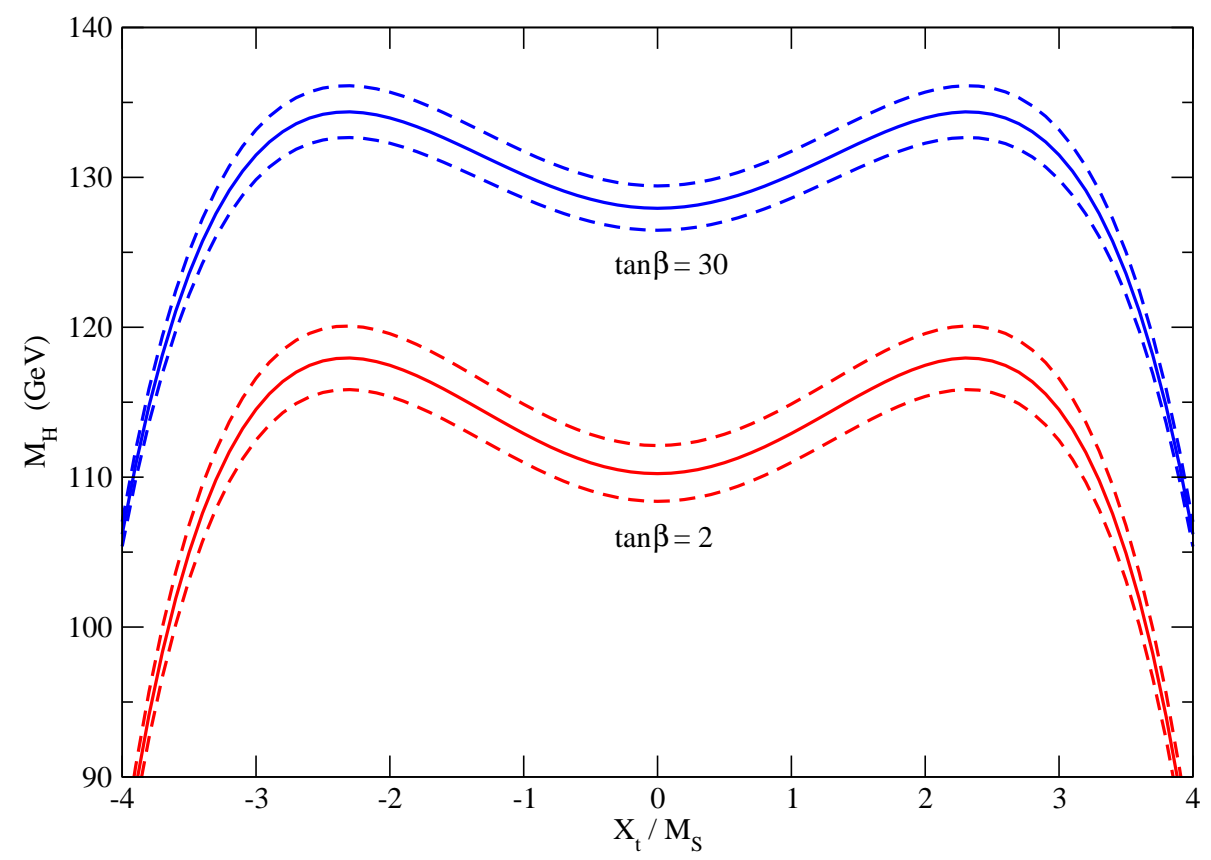

Figure 2: The Higgs boson mass as a function of the ratio $X_{t} / M_{S}$ for $M_{S}=10 \mathrm{TeV}$, $\mu=m_{1 / 2}=500 \mathrm{GeV}$ and two values of $\tan \beta$. The dashed lines correspond to a $\pm 1 \sigma$ variation of the top quark mass. 


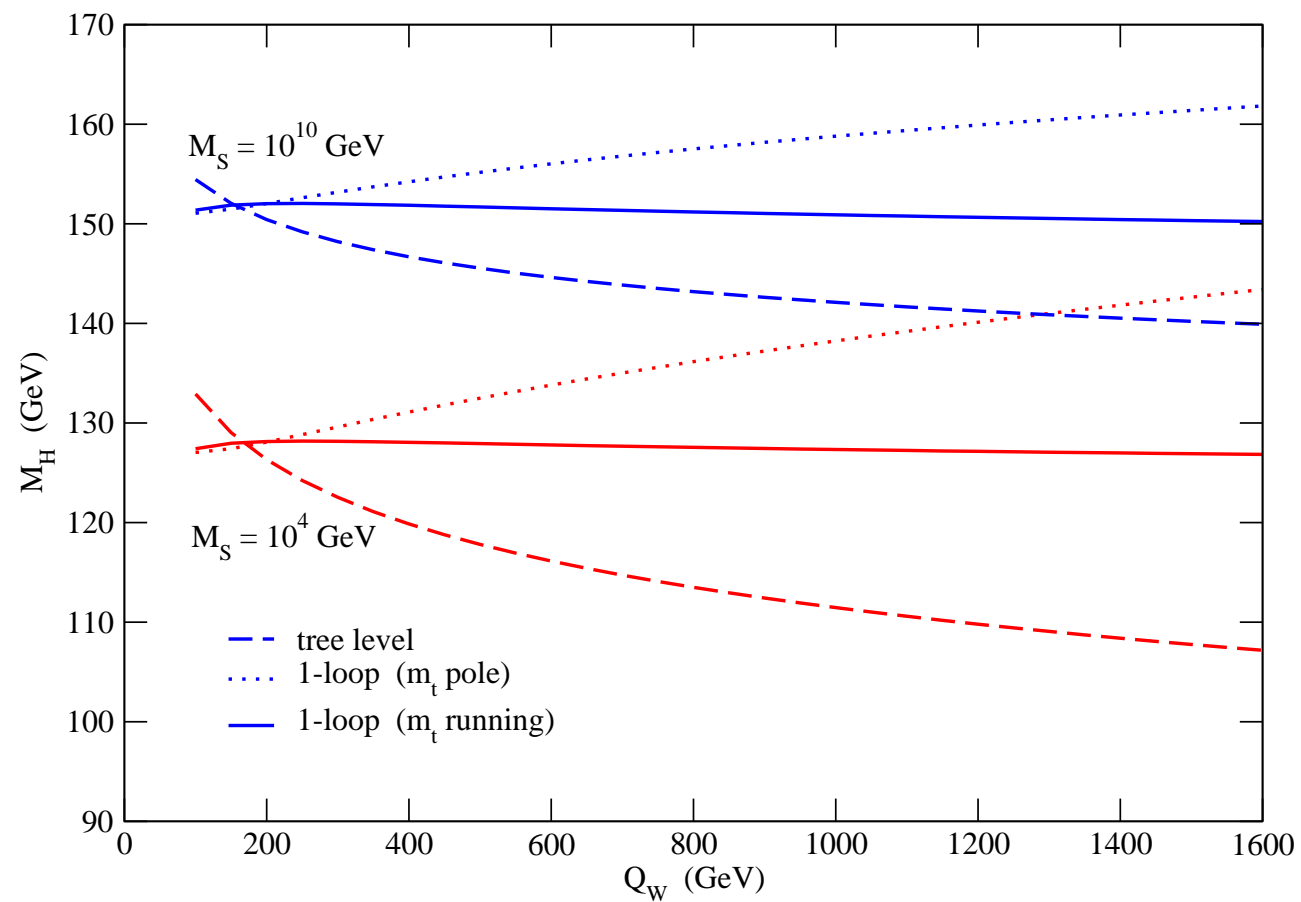

Figure 3: The Higgs boson mass as a function of the scale $Q_{\mathrm{w}}$ in various approximations for $M_{S}=10^{4}$ and $10^{10} \mathrm{GeV}$. For the meaning of the different curves see the text.

Fig. 2 shows the dependence of $M_{H}$ on the stop mixing term $X_{t}$, for $M_{S}=10 \mathrm{TeV}$ and the other SUSY parameters chosen as in fig. 1. It can be seen that, for not too large values of $X_{t} / M_{S}$, the dependence of $M_{H}$ on $X_{t}$ is milder than in the usual MSSM plots with stop masses of the order of $1 \mathrm{TeV}$ (see, e.g., fig. 1.4 of the second paper in ref. [26]). This is due to the fact that the threshold correction to the Higgs quartic coupling in eq. (6) is computed in terms of the running top Yukawa coupling $h_{t}\left(M_{S}\right)$, and the RG evolution up to the large scale $M_{S}$ has the effect of suppressing $h_{t}$.

It is also useful to investigate how the one-loop result for $M_{H}$ depends on the renormalization scale $Q_{\mathrm{w}}$ at which we stop the $\mathrm{RG}$ evolution of the Lagrangian parameters and compute the physical mass of the Higgs boson. In an ideal, all-loop calculation the result for the physical mass would not depend at all on the choice of such scale. However, since we are truncating our calculation at the one-loop order there will be a residual scale dependence, that we can take as a lower bound on (but not necessarily as a full estimate of) the uncertainty associated with higher-order corrections. In fig. 3 we show the dependence of $M_{H}$ on the scale $Q_{\mathrm{w}}$ for the two choices of the heavy scalar mass $M_{S}=10^{4} \mathrm{GeV}$ and $M_{S}=10^{10}$ $\mathrm{GeV}$ (the other relevant parameters are chosen as $\tan \beta=30, A_{t}=0, \mu\left(M_{Z}\right)=m_{1 / 2}=500$ $\mathrm{GeV})$. In each set of curves the dashed line represents the tree-level result, as in eq. (7) with $\delta^{\mathrm{SM}}$ and $\delta^{\chi}$ set to zero; the dotted line represents the one-loop result in which $\delta^{\mathrm{SM}}$ is expressed in terms of the physical top mass $M_{t}$; the solid line represents the one-loop result in which $\delta^{\mathrm{SM}}$ is expressed in terms of the running top mass $m_{t}\left(Q_{\mathrm{w}}\right)$ (as is done in figs. 1 and 2). It can be seen that the tree-level result shows a marked dependence on $Q_{\mathrm{w}}$, due to the 
scale dependence of $\lambda$ in eq. (77). A non-negligible dependence is also present in the one-loop result based on the physical $M_{t}$. On the other hand, the residual scale dependence is very small when the running top mass is used in the one-loop correction. This is reminiscent of the situation in the MSSM, where the use of the physical top mass in the one-loop corrections leads to an excessively high estimate of the light Higgs boson mass, compensated for by large negative two-loop corrections, whereas the two-loop corrections are much smaller when the running top mass is used in the one-loop part. We also note that the three determinations of $M_{H}$ in fig. 3 are in good agreement with each other for the particular choice $Q_{\mathrm{w}} \approx M_{t}$.

The tree-level chargino and neutralino mass matrices read

$$
\mathcal{M}_{C}=\left(\begin{array}{cc}
M_{2} & \tilde{g}_{u} v \\
\tilde{g}_{d} v & \mu
\end{array}\right), \quad \mathcal{M}_{N}=\left(\begin{array}{cccc}
M_{1} & 0 & -\frac{\tilde{g}_{d}^{\prime} v}{\sqrt{2}} & \frac{\tilde{g}_{u}^{\prime} v}{\sqrt{2}} \\
0 & M_{2} & \frac{\tilde{g}_{d} v}{\sqrt{2}} & -\frac{\tilde{g}_{u} v}{\sqrt{2}} \\
-\frac{\tilde{g}_{d}^{\prime} v}{\sqrt{2}} & \frac{\tilde{g}_{d} v}{\sqrt{2}} & 0 & -\mu \\
\frac{\tilde{g}_{u}^{\prime} v}{\sqrt{2}} & -\frac{\tilde{g}_{u} v}{\sqrt{2}} & -\mu & 0
\end{array}\right) .
$$

The values of the tree-level chargino $\left(\chi_{1,2}^{+}\right)$and neutralino $\left(\chi_{1,2,3,4}^{0}\right)$ masses depend on the renormalization scale at which the various parameters in eq. (9) are computed. To reduce this scale dependence we include the one-loop corrections to the chargino and neutralino masses, once again adapting the MSSM formulae of ref. [14] to the Split-SUSY case (see the appendix for details). In figs. 4 and 5 we show the masses of charginos and neutralinos, respectively, as function of the scale $Q_{\mathrm{w}}$, for $M_{S}=10^{4} \mathrm{GeV}, \tan \beta=30$ and $\mu\left(M_{Z}\right)=m_{1 / 2}=500 \mathrm{GeV}$. In each plot the dashed lines represent the tree-level result and the solid lines represent the one-loop result. It can be seen that the inclusion of the radiative correction improves the scale dependence of the masses of charginos and neutralinos. For the choice of parameters considered in figs. 4 and 5 the only exception is the mass of the lightest neutralino, which is mostly bino: the tree-level value of $m_{\chi_{1}^{0}}$ has already very little dependence on the scale, and the one-loop corrections are negligible.

Finally, the physical gluino mass $M_{\tilde{g}}$ is related to the $\overline{\mathrm{MS}}$ parameter $M_{3}$ by

$$
M_{\tilde{g}}=M_{3}(Q)\left[1+\frac{\alpha_{s}}{4 \pi}\left(12+9 \log \frac{Q^{2}}{M_{3}^{2}}\right)\right] .
$$

In the analysis of the constraints on the Split-SUSY parameter space that we present in sections 3 and 4 we will be mostly interested in phenomena that involve charginos and neutralinos. For this reason we find it convenient to choose by default a value of $Q_{\mathrm{w}}$ that is representative of the masses in the chargino/neutralino sector, i.e. $Q_{\mathrm{W}}=\sqrt{\mu M_{2}}$. While a priori this might not be the best choice of renormalization scale for phenomena involving the Higgs boson, fig. 3 shows that the Higgs boson mass is not significantly affected by the choice of $Q_{\mathrm{w}}$ when we compute the one-loop corrections in terms of the running top mass. On the other hand, in many models of SUSY breaking the gluino can be quite heavier than the other gauginos, thus we will choose $Q_{\mathrm{w}}=M_{3}$ when discussing the gluino mass and decays. 


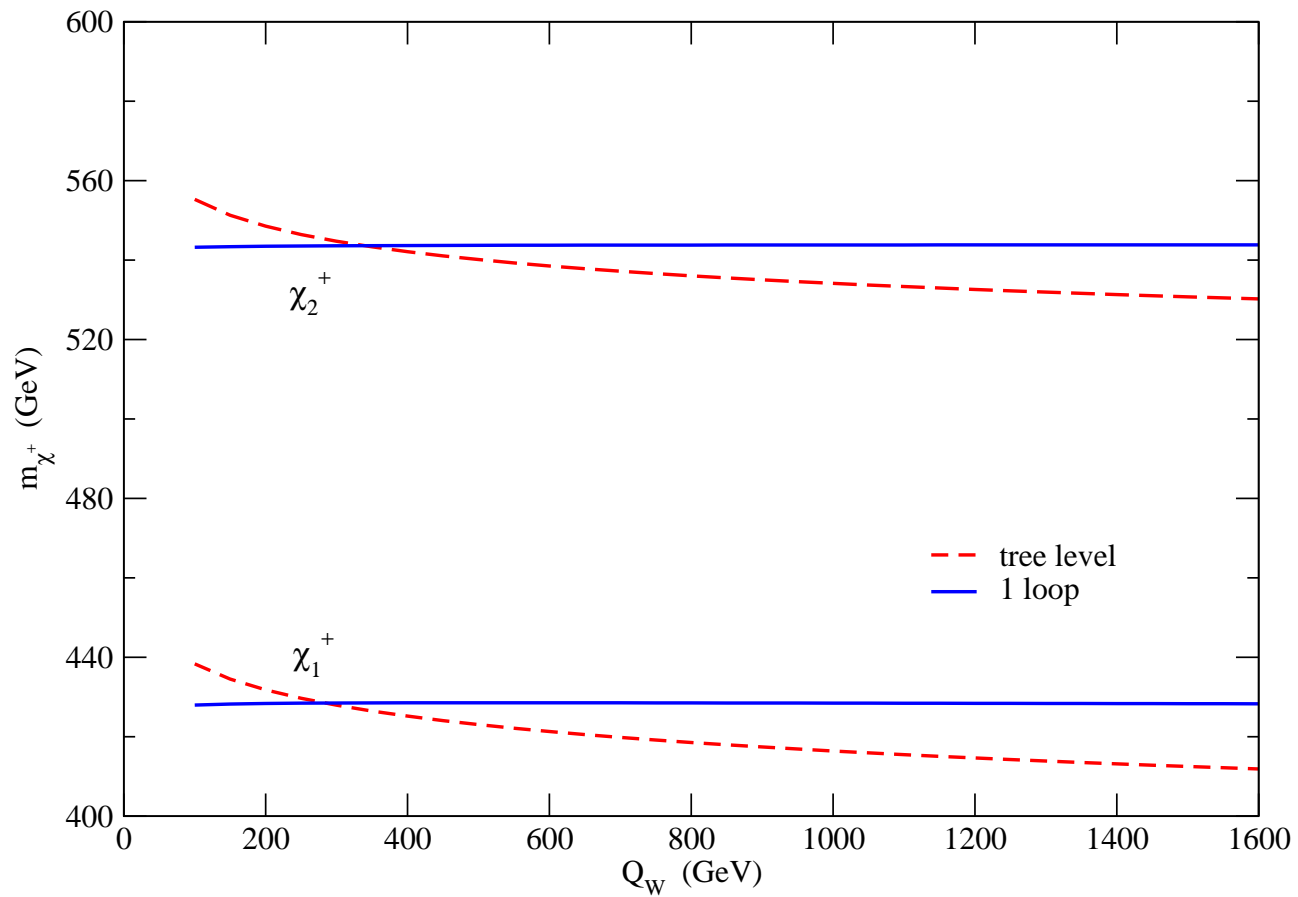

Figure 4: The chargino masses as a function of the scale $Q_{\mathrm{w}}$ for $M_{S}=10^{4} \mathrm{GeV}, \tan \beta=$ 30, $\mu=m_{1 / 2}=500 \mathrm{GeV}$. The dashed lines are tree-level results and the solid lines are one-loop results.

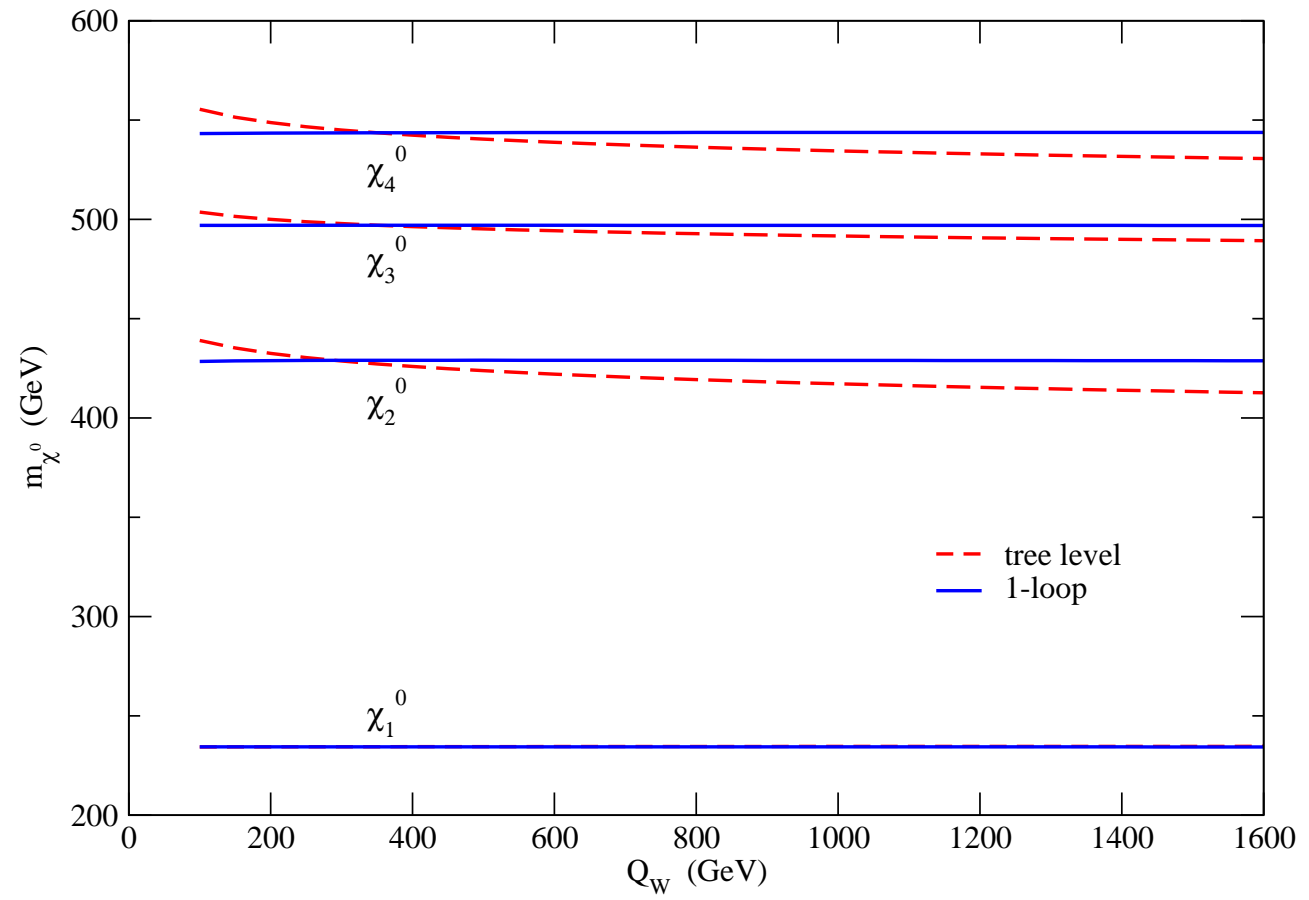

Figure 5: Same as fig. 4 for the four neutralino masses. 


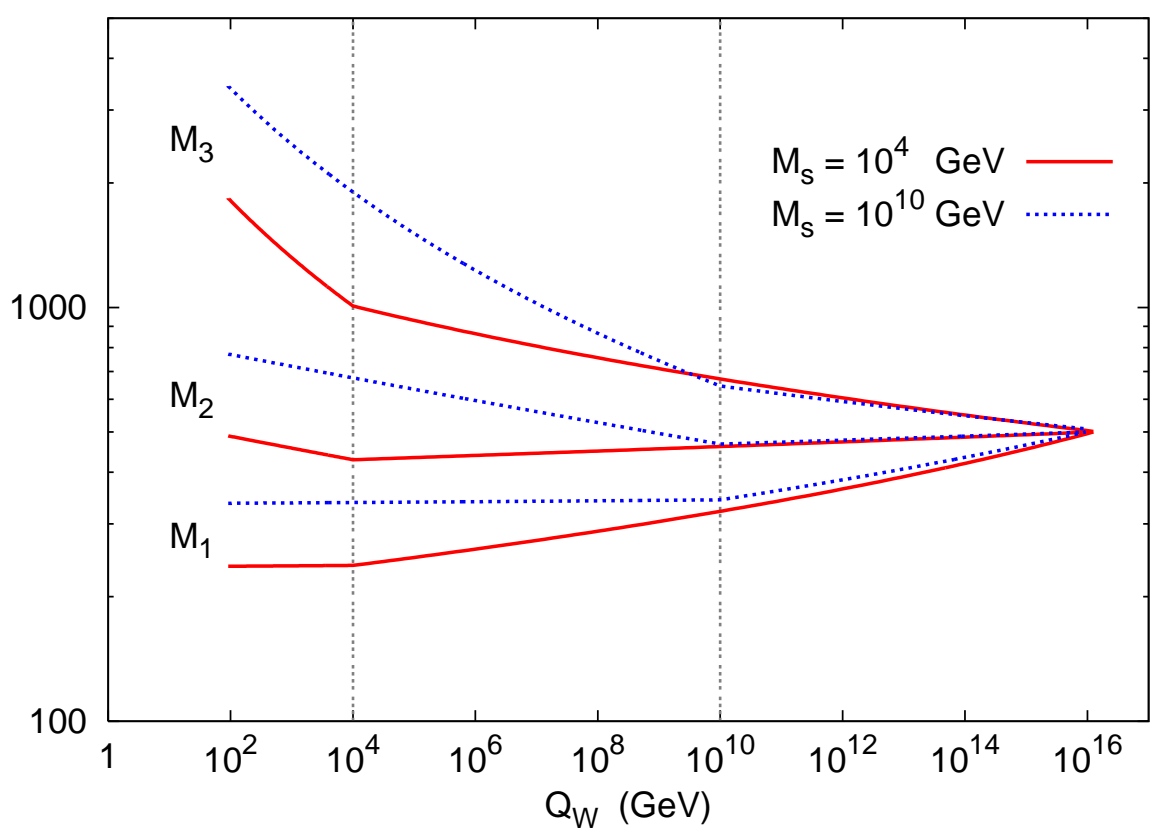

Figure 6: The evolution of the gaugino mass parameters from the GUT scale to the weak scale in the universal scenario for $M_{S}=10^{4}$ and $10^{10} \mathrm{GeV}$.

\subsection{Patterns of soft SUSY-breaking gaugino masses}

The soft SUSY-breaking gaugino mass parameters $M_{1,2,3}$ entering the chargino, neutralino and gluino masses are determined via one loop RGEs once their values at the GUT scale are fixed. If one assumes universality of these parameters, $M_{1}=M_{2}=M_{3} \equiv m_{1 / 2}$ at $M_{\mathrm{Gut}}$, as is done e.g. in the minimal Supergravity (mSUGRA) model [18, the ratios between the weak scale values are simply related to the squares of the gauge coupling constants $\alpha_{i} \equiv g_{i}^{2} /(4 \pi)$ by $M_{1}: M_{2}: M_{3}=\alpha_{1}: \alpha_{2}: \alpha_{s}$. These values also depend on the intermediate scale $M_{S}$ below which the contributions of the sfermions and of the heavy Higgs doublet are decoupled from the RGEs. The evolution of $M_{1,2,3}$ is displayed in fig. 6, where a common GUT value $m_{1 / 2}=500 \mathrm{GeV}$ has been assumed and two values for the scalar masses, $M_{S}=10^{4} \mathrm{GeV}$ and $M_{S}=10^{10} \mathrm{GeV}$, have been used. At the scale $M_{Z}$ one obtains the ratios $M_{1}: M_{2}: M_{3}=$ $1.0: 2.0: 7.8$ for $M_{S}=10^{4} \mathrm{GeV}$, while one has $M_{1}: M_{2}: M_{3}=1.0: 2.3: 10.2$ for $M_{S}=10^{10}$ $\mathrm{GeV}$. If the scale $M_{S}$ had been set to $1 \mathrm{TeV}$, as in the usual MSSM with scalar masses of the same order as the gaugino masses, one would have obtained $M_{1}: M_{2}: M_{3} \simeq 1: 2: 7$.

Since for heavy scalars the number of basic input parameters of the model is rather small, one can relax the assumption of a universal gaugino mass at the GUT scale and still have a rather predictive model which, in many instances, could lead to a rather different phenomenology. In this paper, rather than performing a general scan with 4 or 5 input parameters (i.e. $M_{1}, M_{2}, M_{3}, \mu$ and possibly $M_{S}$ ) we will consider two specific SUSY models 
in which the boundary conditions for the gaugino masses at the GUT scale are different from those of the universal scenario. This will simplify our numerical analysis and, at the same time, allow us to address the new interesting phenomenology induced by the non-universality of the gaugino masses.

The first scenario that we consider is a gravity-mediated SUSY-breaking scenario in which, to lowest order, the gaugino masses arise from a dimension-5 operator

$$
\mathcal{L} \propto \frac{\left\langle F_{\Phi}\right\rangle_{a b}}{M_{\text {Planck }}} \cdot \lambda^{a} \lambda^{b},
$$

where $\lambda^{a, b}$ are the gaugino fields and $F_{\Phi}$ the auxiliary component of a left-handed chiral superfield $\Phi$ which couples to the SUSY field strength. In the usual mSUGRA model with SU(5) grand unification the SUSY-breaking field $F_{\Phi}$ is a singlet under the unifying gauge group, leading to universal gaugino masses. However, the chiral superfield $\Phi$ can sit in any representation of the symmetric product of the adjoint group [20]. In the case of SU(5) symmetry, $F_{\Phi}$ could belong to an irreducible representation which results from the symmetric product of two adjoints

$$
(24 \otimes 24)_{\text {symmetric }}=1 \oplus 24 \oplus 75 \oplus 200
$$

Once the neutral component of $F_{\Phi}$ has acquired a vacuum expectation value, $\left\langle F_{\Phi}\right\rangle_{a b}=$ $V_{a} \delta_{a b}$, the vevs $V_{a}$ determine the relative magnitude of the soft SUSY-breaking gaugino mass parameters $M_{a}$ at the GUT scale [19]. These are shown in the left-hand side of table 1 and, as can be seen, only in the singlet case $\mathbf{1}$ are these parameters universal.

Another set of scenarios that we will consider are four-dimensional string models in which the source of SUSY breaking resides predominantly in the moduli sector. In particular, in the orbifold OII scenario in which all chiral fields have modular weight equal to unity, the boundary conditions for the gaugino mass parameters at the GUT scale are [21]

$$
M_{a} \approx \pm 10^{-3}\left(b_{a}+\delta_{G S}\right) m_{3 / 2}, \quad a=1,2,3
$$

where $m_{3 / 2}$ is the gravitino mass, $b_{a}$ the coefficient of the MSSM beta function for the gauge coupling constant $g_{a}$, and $\delta_{G S}$ the Green-Schwartz mixing parameter which is a negative integer number in this case. In these scenarios the scalars, with masses $m_{0}^{2} \approx 10^{-3}\left(-\delta_{G S}\right) m_{3 / 2}^{2}$, are expected to be much heavier than the gauginos and the pattern of gaugino masses, compared to the universal case, is driven by the parameter $\delta_{G S}$. For the choice $\delta_{G S}=-4$ one obtains at $M_{\mathrm{GUT}}$ the mass pattern $M_{1}: M_{2}: M_{3} \sim \frac{53}{5}: 5: 1$; see table 1 .

Other mass patterns can be obtained by choosing different values of the integer parameter $\delta_{G S}$, and some of them are in fact similar to those of the non-singlet representation models shown in table 1. For instance, for $\delta_{G S}=-2$, one obtains the ratios $M_{1}: M_{2}: M_{3} \sim \frac{43}{5}: 3:-1$ which are close to those of the scenario 200. In the orbifold OI-type scenario [21], which differs from the previous one in the fact that the modular weights are not all equal to unity, 


\begin{tabular}{|c||c|c|c|}
\hline & $Q=M_{\text {GUT }}$ & $Q=M_{Z}\left[M_{S}=10^{4} \mathrm{GeV}\right]$ & $Q=M_{Z}\left[M_{S}=10^{10} \mathrm{GeV}\right]$ \\
\hline $\mathbf{1}$ & $1: 1: 1$ & $1.0: 2.0: 7.8$ & $1.0: 2.3: 10.2$ \\
\hline $\mathbf{2 4}$ & $1: 3:-2$ & $1.0: 6.3:-15.2$ & $1.0: 6.9:-19.7$ \\
\hline $\mathbf{7 5}$ & $5:-3:-1$ & $1.0:-1.2:-1.5$ & $1.0:-1.4:-2.0$ \\
\hline $\mathbf{2 0 0}$ & $10: 2: 1$ & $2.4: 1.0: 1.9$ & $2.2: 1.0: 2.2$ \\
\hline \hline OII & $53 / 5: 5: 1$ & $1.4: 1.3: 1.0$ & $1.0: 1.1: 1.0$ \\
\hline
\end{tabular}

Table 1: The ratios of gaugino mass parameters, $M_{1}: M_{2}: M_{3}$, at the renormalization scales $M_{\mathrm{GUT}}, M_{Z}$ (with $M_{S}=10^{4} \mathrm{GeV}$ ) and again $M_{Z}$ (with $M_{S}=10^{10} \mathrm{GeV}$ ), for the different patterns of soft SUSY breaking.

one could obtain different gaugino mass ratios. However, in many cases, the pattern is similar to that of the scenarios discussed above and, for instance, ratios that are close to those of the scenario 75 can be obtained for $\delta_{G S}=-5$.

The relations between the gaugino masses $M_{1,2,3}$ at the scale $M_{\mathrm{GUT}}$ and at the weak scale $M_{Z}$ are summarized in table 1 for the different scenarios. We have used the one-loop RGEs for the couplings and assumed a common scalar mass $M_{S}$ of either $10^{4} \mathrm{GeV}$ or $10^{10} \mathrm{GeV}$. As can be seen, the pattern of the gaugino mass parameters at $M_{Z}$ (and hence the gluino, neutralino and chargino masses) can be quite different from the universal case (scenario 1 ) in which for $M_{S}=10^{4} \mathrm{GeV}$ one has approximately $M_{1}: M_{2}: M_{3}=\alpha_{1}: \alpha_{2}: \alpha_{s} \simeq 1: 2: 8$.

In particular, in the scenario 200 where $M_{2}<M_{1}$, the LSP is wino-like for large values of the parameter $\mu$, implying that $\chi_{1}^{0}$ and $\chi_{1}^{ \pm}$are nearly degenerate in mass. Again for large $\mu$ values, the neutralinos $\chi_{1}^{0}$ and $\chi_{2}^{0}$ and the charginos $\chi_{1}^{ \pm}$masses are very close to each other in the scenario $\mathbf{7 5}$ since $\left|M_{1}\right| \sim\left|M_{2}\right|$, while in the scenario $\mathbf{2 4}$ the mass splitting between the LSP and the states $\chi_{2}^{0}$ and $\chi_{1}^{ \pm}$can be very large since $M_{2} \sim 6 M_{1}$. Finally, in the OII model one has $M_{3}<M_{1}, M_{2}$ and the gluino tends to be the LSP unless $\mu$ is very small, in which case $\chi_{1}^{0}, \chi_{2}^{0}$ and $\chi_{1}^{+}$are higgsino-like and almost degenerate in mass. Note that, in general, the weak-scale ratios among the three gaugino masses show a dependence on the scale $M_{S}$ at which the sfermions are integrated out.

There are many other SUSY models that lead to non-universal gaugino masses, and a review has recently been given in ref. [27]. However, in most cases one is very close in practice to the patterns that have been introduced above, and the phenomenology of the gaugino sector is quite similar. For instance, the weak-scale pattern of gaugino masses that emerges from anomaly mediated SUSY-breaking (AMSB) models [28], $M_{1}: M_{2} \sim 3: 1$, is similar to that of the model 200. In mirage gaugino mediation [29], where SUSY breaking is realized in higher-dimensional brane models (a scheme that is realized naturally in the 
so-called KKLT-type moduli stabilization models [30]), one has [27] $M_{1}: M_{2} \sim 1: 1.3$ which, again, is similar to the pattern of the scenario 75. Thus, we believe that the scenarios discussed above, with the patterns of gaugino masses of table 1, are representative of a wide spectrum of non-universal models.

\subsection{Implementation of the MSSM with heavy scalars in SuSpect}

We have implemented this MSSM scenario with heavy scalars into the RGE code SuSpect [11. This model can be chosen by selecting at the very beginning of the input file suspect 2 . in or alternatively suspect2_lha.in, the option SHeavy by putting ichoice(1) = 3. The only two sets of basic input parameters needed to be set are:

- The SM basic input parameters, i.e. the electromagnetic, strong and weak couplings, the $Z$ boson mass and the third-family fermion masses:

$$
\alpha\left(M_{Z}\right), \alpha_{s}\left(M_{Z}\right), G_{F}, M_{Z}, M_{t}, m_{b}\left(m_{b}\right), M_{\tau}
$$

- The additional input parameters specific for this model:

$$
\mu\left(M_{Z}\right), M_{1}\left(M_{\mathrm{GUT}}\right), M_{2}\left(M_{\mathrm{GUT}}\right), M_{3}\left(M_{\mathrm{GUT}}\right), M_{S}, \tan \beta\left(M_{S}\right), A_{t}\left(M_{S}\right) .
$$

All the other parameters of these files are irrelevant.

The routine SHeavy.f performs the RG evolution for the gauge couplings, the third generation fermion Yukawa couplings, the gaugino-higgsino-Higgs boson couplings $\left(g_{u, d}, \tilde{g}_{u, d}\right)$, the gaugino mass parameters, the $\mu$ parameter, and the quartic Higgs coupling $\lambda$. This routine also contains all the relevant one-loop radiative corrections.

The output file contains the physical chargino and neutralino masses and the elements of the mixing matrices $U, V$ and $N$ (see the appendix for details), as well as the physical masses of the gluino and of the lightest Higgs boson. They are computed at a scale $Q_{W}$ set by default to $Q_{W}=\sqrt{\mu M_{2}}$, except for the gluino mass which is computed at the scale $M_{3}$. The masses of all the sfermions and of the heavy Higgs bosons are considered to be degenerate and set to $M_{S}$, while the various mixing angles (in the third-generation-sfermion and Higgs sectors) are set to zero.

Note that, for the phenomenological analyses that will be presented in the next two sections, we also needed to adapt to the Split-SUSY scenario the two programs HDECAY [16] and SDECAY [17], which compute the decay widths and branching ratios of the MSSM Higgs bosons and of the SUSY particles, respectively. These programs use the output given by SuSpect for the soft SUSY-breaking parameters, the mixing matrix elements and the sparticle and Higgs masses, but they calculate internally the various couplings. In particular, the Higgs couplings to neutralinos and charginos (which are different from the usual MSSM case) are hard-coded and need to be adapted. 


\section{Collider and Dark Matter constraints}

In this section we analyze the constraints on the MSSM with heavy scalars, first from collider searches and high-precision data [22] and then from cosmological data, in particular the relic density measurement of DM by the WMAP satellite [31] and the gluino lifetime [32 34]. A special attention will be given to the non-universal gaugino mass scenarios discussed in the previous section, where several new features compared to the universal case appear. The DM and some collider constraints for non-universal gaugino masses have been discussed in ref. [35] in the usual MSSM with light scalars, while some implications of DM in the Split-SUSY scenario have been studied in Refs. [15, 36].

\subsection{Constraints from collider data}

In the scenario in which a universal gaugino mass $m_{1 / 2}$ is assumed at the GUT scale, leading to the approximate relation $M_{1}: M_{2}: M_{3} \sim 1: 2: 8$ at the weak scale, the strongest experimental bound is due to the negative search of charginos at LEP2 up to energies of $\sqrt{s} \simeq 208 \mathrm{GeV}$. From pair production of the lightest chargino, $e^{+} e^{-} \rightarrow \chi_{1}^{ \pm} \chi_{1}^{\mp}$, one obtains the mass bound 22 ]

$$
m_{\tilde{\chi}_{1}^{ \pm}} \gtrsim 103 \mathrm{GeV} \text {. }
$$

The chargino mass bound in eq. (14) is valid only if the mass splitting between the lightest chargino and neutralino is large enough, $\Delta M \equiv m_{\chi_{1}^{ \pm}}-m_{\chi_{1}^{0}} \gtrsim$ a few GeV. For small $\Delta M$ values, as is the case when the LSP neutralino is higgsino- or wino-like, $m_{\chi_{1}^{ \pm}} \simeq m_{\chi_{1}^{0}} \simeq|\mu|$ or $M_{2}$, the bound becomes weaker and, for very heavy scalar fermions, one has $m_{\tilde{\chi}_{1}^{ \pm}} \gtrsim 92 \mathrm{GeV}$ [22]. Using the weak-scale gaugino mass relations above, the bound on the lightest chargino mass translates into a lower bound on the LSP mass, $m_{\tilde{\chi}_{1}^{0}} \gtrsim 50 \mathrm{GeV}$ if the LSP neutralino is bino-like, in which case one has $m_{\chi_{1}^{0}} \simeq M_{1} \simeq \frac{1}{2} M_{2} \simeq \frac{1}{2} m_{\chi_{1}^{ \pm}} ; \chi_{1}^{0}$ is thus too heavy to be kinematically accessible in invisible $Z$ boson decays at LEP1, $Z \rightarrow \chi_{1}^{0} \chi_{1}^{0}$. In the higgsino- and wino-like regions for the LSP neutralino the mass bound is higher, $m_{\tilde{\chi}_{1}^{0}} \gtrsim 92 \mathrm{GeV}$, as $\chi_{1}^{0}$ is almost degenerate with the chargino $\chi_{1}^{ \pm}$as discussed above. Furthermore, the constraint on $M_{2}$ from the bound on $m_{\chi_{1}^{ \pm}}$translates into a constraint on the gluino mass, $m_{\tilde{g}} \sim M_{3} \gtrsim 350$ $\mathrm{GeV}$, which is higher than the direct bound from Tevatron searches when scalar quarks are very heavy [22]

$$
m_{\tilde{g}} \gtrsim 195 \mathrm{GeV}
$$

The constraints on the $\left[M_{2}, \mu\right]$ parameter space from LEP2 and Tevatron negative searches of SUSY particles are summarized in fig. 7. Here and in the following $M_{2}$ and $\mu$ have to be interpreted as $\overline{\mathrm{MS}}$ parameters evaluated at the renormalization scale $Q=Q_{\mathrm{w}}$. Besides the process $Z \rightarrow \chi_{1}^{0} \chi_{1}^{0}$, which contributes to the invisible $Z$ boson decay that is tightly constrained by LEP1 data, $\Gamma_{Z}^{\text {inv }} \lesssim 2 \mathrm{MeV}[22$, we have imposed that the cross sections for 


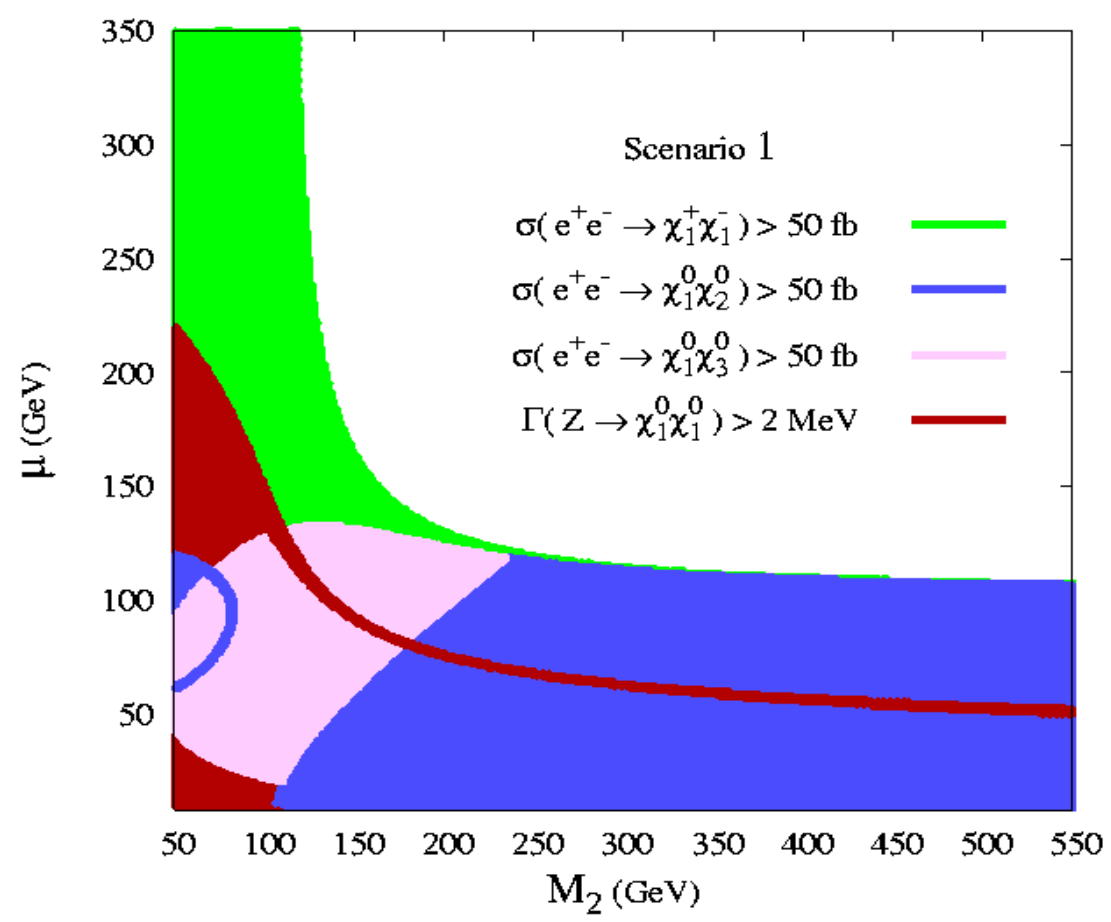

Figure 7: Constraints on the $\left[M_{2}, \mu\right]$ parameter space for the scenario 1 with universal gaugino masses at the GUT scale and with $M_{S}=10^{4} \mathrm{GeV}, A_{t}=0$ and $\tan \beta=30$; the different colors correspond to the regions excluded by the LEP bounds on the partial decay width $\Gamma\left(Z \rightarrow \chi_{1}^{0} \chi_{1}^{0}\right)$ and on the production cross sections $e^{+} e^{-} \rightarrow \chi_{1}^{ \pm} \chi_{1}^{\mp}, \chi_{1}^{0} \chi_{2}^{0}, \chi_{1}^{0} \chi_{3}^{0}$.

the three processes $e^{+} e^{-} \rightarrow \chi_{1}^{ \pm} \chi_{1}^{\mp}, e^{+} e^{-} \rightarrow \chi_{1}^{0} \chi_{2}^{0}$ and $e^{+} e^{-} \rightarrow \chi_{1}^{0} \chi_{3}^{0}$ are smaller than $50 \mathrm{fb}$ which, given the collected luminosity of $\mathcal{L} \sim 100 \mathrm{pb}^{-1}$ at the c.m. energy $\sqrt{s} \sim 208 \mathrm{GeV}$, corresponds to less than 5 events. As can be seen, the strongest constraint is by far due to eq. (14), i.e. the green (light grey) area. The process $e^{+} e^{-} \rightarrow \chi_{1}^{0} \chi_{2}^{0}$ where the LSP and the next-to-lightest neutralino are pair produced, although more favored by phase space, does not add much information as the cross section is generally much smaller. In the small blue (dark grey) oval region on the left of the plot $\chi_{2}^{0}$ is mostly wino, while in the region with large $M_{2}$ and small $\mu$ on the right of the plot $\chi_{2}^{0}$ is mostly higgsino.

All these bounds can be adapted to scenarios in which the boundary conditions for the gaugino masses at the high scale are different. However, in some cases, their impact can be widely different compared to the universal scenario, as shown in fig. 8 where the constrained $\left[M_{2}, \mu\right]$ parameter space with $M_{S}=10^{4} \mathrm{GeV}$ is displayed for the four scenarios 24, 75, 200 and OII. We have assumed $\mu>0$ but a similar pattern is obtained for $\mu<0$.

In the non-universal scenario 24 one has $M_{2}: M_{1} \sim 6: 1$ for the wino and bino masses at the weak scale and, as in the scenario 1 , the chargino mass bound in eq. (14) leads to the strongest constraint and rules out the entire $\mu, M_{2} \gtrsim 100 \mathrm{GeV}$ range. For large values of the wino mass, $M_{2} \gtrsim 300 \mathrm{GeV}$, and small values of $\mu$, the phase space for the process 
$e^{+} e^{-} \rightarrow \chi_{1}^{0} \chi_{3}^{0}$ is still open and a small additional region is ruled out. The process where the second neutralino is produced in association with the LSP, $e^{+} e^{-} \rightarrow \chi_{1}^{0} \chi_{2}^{0}$, also plays a role at even larger values of $M_{2}$ and rules out another portion of the parameter space. If the lighter chargino and neutralinos are gaugino-like, $M_{2}, M_{1} \ll|\mu|$, the LSP neutralino is bino-like and the chargino mass bound in eq. (14) translates into the relatively weak bound $m_{\chi_{1}^{0}} \gtrsim 17 \mathrm{GeV}$. This opens the possibility that the decay mode $Z \rightarrow \chi_{1}^{0} \chi_{1}^{0}$ contributes to the invisible decay of the $Z$ boson. For moderate values of $\mu$, for which the LSP has a higgsino component and hence sizable couplings to the $Z$ boson, the constraint $\Gamma_{\text {inv }} \lesssim 2 \mathrm{MeV}$ rules out a region in the $\left[M_{2}, \mu\right]$ plane that is not excluded by any other process.

In the scenario $\mathbf{7 5}$ the masses of the lightest chargino and of the two lightest neutralinos are very close to each other, since $M_{1}:\left|M_{2}\right| \sim 1: 1.2$. Therefore, chargino pair production, which has the largest cross section among the three processes with a similar phase space $e^{+} e^{-} \rightarrow \chi_{1}^{ \pm} \chi_{1}^{\mp}, \chi_{1}^{0} \chi_{2}^{0}$ and $\chi_{2}^{0} \chi_{2}^{0}$, leads to the strongest constraint on the $\left[M_{2}, \mu\right]$ parameter space. However, since the weak-scale value of $M_{3}$ is also close to the values of $M_{1}$ and $M_{2}$, the constraint $m_{\tilde{g}}>195 \mathrm{GeV}$ from negative searches of gluinos at the Tevatron plays an important role, in contrast to the scenarios 1 and 24 in which $M_{3} \gtrsim 3-4 M_{2}$ and the bound of eq. (15) is superseded by that of eq. (14). Since $M_{3} \sim 1.3 M_{2}$, the entire area $M_{2} \gtrsim 150$ $\mathrm{GeV}$ is ruled out by eq. (15) independently of the value of $\mu$.

A similar pattern occurs in the scenario 200 where the mass parameters $M_{1}, M_{2}$ and $M_{3}$ are also close to each other. However, since here the ratio $M_{3} / M_{2}$ is larger than in the scenario $\mathbf{7 5}$, the chargino mass bound is stronger than the bound from gluino searches. Note also that, in this scenario, the LSP is wino-like for large values of $\mu$ and the chargino mass bound translates to $m_{\chi_{1}^{0}} \gtrsim 100 \mathrm{GeV}$ for the LSP and the two particles are almost degenerate in mass (this also holds in the higgsino-like region for the LSP).

Finally, the string-inspired scenario OII is interesting in many respects. As in the universal scenario 1 chargino pair production is the most constraining of all LEP production processes and rules out the same area of the $\left[M_{2}, \mu\right]$ parameter space. However, since here the smallest of the gaugino masses is the gluino mass $M_{3}$, the Tevatron bound in eq. (15) rules out a significant portion of the parameter space, namely $M_{2} \lesssim 230 \mathrm{GeV}$. Furthermore, the gluino tends to be the LSP for large values of $\mu$ when the lightest neutralino is gauginolike. A strongly interacting stable particle is disfavored by cosmological data, therefore this area of the parameter space has to be excluded.

Another important collider constraint comes from the negative search of Higgs bosons at LEP2 where a lower bound, $M_{H} \gtrsim 114 \mathrm{GeV}$, has been set on the mass of a SM-like Higgs boson [22]. As previously discussed, the Higgs boson in the MSSM with heavy scalars is SM-like but its mass is generally larger than $130 \mathrm{GeV}$ (for $M_{S} \gtrsim 10^{4} \mathrm{GeV}$ and $\tan \beta$ large enough), therefore this constraint does not apply in our case. Note, however, that there is a chance that such a Higgs particle could be observed at the Tevatron, either in the processes $q q \rightarrow H W \rightarrow b \bar{b} \ell \nu$ for $M_{H} \sim 130 \mathrm{GeV}$ or in the process $g g \rightarrow H \rightarrow W W^{(*)}$ for $M_{H} \sim 160$ $\mathrm{GeV}$, if a large integrated luminosity is collected; see for instance ref. [26] for details. 

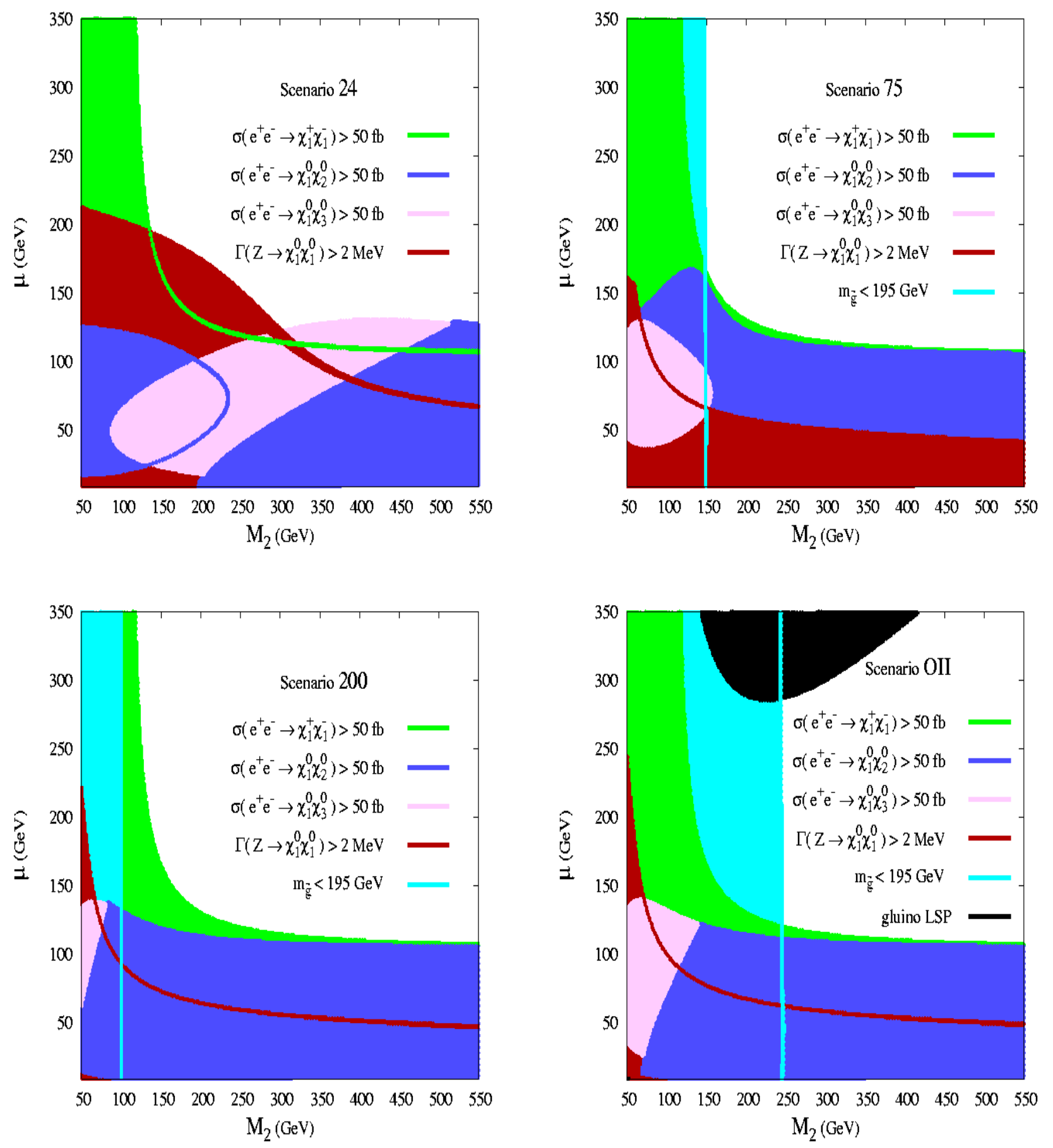

Figure 8: The same as in fig. 7 but for the non-universal scenarios. Where relevant, the effect of the gluino mass bound from Tevatron searches is also shown. 
Finally, we summarize the constraints from high-precision data and rare decays. Because the sfermions are very heavy, the SUSY-particle contributions to the anomalous magnetic moment $(g-2)_{\mu}$ of the muon (which occur essentially through smuon-neutralino and sneutrino-chargino loops, with possible very small contributions from Higgs bosons) and to the rare decay of the $b$-quark into a strange quark and a photon $\mathrm{BR}(b \rightarrow s \gamma$ ) (which at leading order occur via loops involving the charged Higgs bosons and top quarks as well as charginos and top squarks) are extremely small, and one is left only with the SM contributions. The effect of SUSY particles on the high-precision electroweak observables measured at LEP, SLC, Tevatron and elsewhere is also very tiny in the case of heavy scalars. Indeed, the dominant contributions to these observables, in particular the $W$ boson mass and the effective weak mixing angle $\sin ^{2} \theta_{W}$, enter via a deviation from unity of the $\rho$ parameter (which measures the relative strength of the neutral to charged current processes at zero momentum transfer). The sfermions and the non-SM Higgs bosons are heavy enough that their contribution to this quantity is negligible. The chargino and neutralino contributions are small because the only terms in the mass matrices that could break the custodial SU(2) symmetry are proportional to $M_{W}$. This has been verified explicitly in the case of universal gaugino masses in ref. [37, where it has been shown that only when charginos and neutralinos have masses very close to the experimental lower bounds can they affect, and only slightly, the electroweak observables.

\subsection{The Dark Matter constraint}

As deduced from the WMAP satellite measurement of the temperature anisotropies in the Cosmic Microwave Background, cold Dark Matter makes up approximately 25\% of the energy of the Universe [31. The DM cosmological density is precisely measured to be

$$
\Omega_{\mathrm{DM}} h^{2}=0.111_{-0.008}^{+0.006}
$$

which leads to $0.088 \leq \Omega_{\mathrm{DM}} h^{2} \leq 0.128$ at the $3 \sigma$ level. The accuracy is expected to be improved to the percent level by future measurements at Planck.

As is well known, the LSP neutralino is an ideal candidate for the weakly interacting massive particle that is expected to form this cold DM [5] and in some areas of the SUSY parameter space the $\chi_{1}^{0}$ cosmological relic density, which is inversely proportional to the neutralino annihilation cross section $\sigma_{\text {ann }} \equiv \sigma\left(\chi_{1}^{0} \chi_{1}^{0} \rightarrow\right.$ SM particles $)$, falls in the range required by WMAP. In the MSSM with heavy scalars, there are essentially only three regions (see later for a fourth possibility) in which this constraint is satisfied:

- The "mixed region" in which the LSP is a higgsino-gaugino mixture, $M_{1} \sim|\mu|$, which enhances (but not too much) its annihilation cross sections into final states containing gauge and/or Higgs bosons and top quarks, $\chi_{1}^{0} \chi_{1}^{0} \rightarrow W^{+} W^{-}, Z Z, H Z, H H$ and $t \bar{t}$. 
- The "pure higgsino" and "pure wino" regions, in which the LSP is almost (but not exactly) degenerate in mass with the lightest chargino and the next-to-lightest neutralino, leading to and enhanced destruction of sparticles since the $\chi_{1}^{+}, \chi_{2}^{0}$ co-annihilation cross sections are much larger than that of the LSP; this solution generally requires LSP masses beyond $1 \mathrm{TeV}$.

- The " $H$-pole" region in which the LSP is rather light, $m_{\chi_{1}^{0}} \sim \frac{1}{2} M_{H}$, and the $s$-channel $H$ exchange is nearly resonant allowing the neutralinos to annihilate efficiently.

The latter scenario has been discussed in the usual MSSM [38] in which the Higgs boson, which has a mass below $\sim 130 \mathrm{GeV}$, decays mostly into $b \bar{b}$ pairs. However, if the common scalar mass $M_{S}$ is very large, as is the case in the present discussion, the Higgs mass will be larger reaching, for instance, the value $M_{H} \sim 150 \mathrm{GeV}$ for $M_{S} \sim 10^{10} \mathrm{GeV}$. In this case the dominant decay mode of the Higgs boson is a pair of $W$ bosons, one being on mass-shell while the other is virtual and decays into two massless fermions, $H \rightarrow W W^{*} \rightarrow W f \bar{f}$.

The partial decay width for this decay is given by 39]

$$
\begin{aligned}
\Gamma\left(H \rightarrow W W^{*}\right) & =\frac{3 G_{\mu}^{2} M_{W}^{4}}{16 \pi^{3}} M_{H}\left[\frac{3\left(1-8 x+20 x^{2}\right)}{(4 x-1)^{1 / 2}} \arccos \left(\frac{3 x-1}{2 x^{3 / 2}}\right)\right. \\
& \left.-\frac{1-x}{2 x}\left(2-13 x+47 x^{2}\right)-\frac{3}{2}\left(1-6 x+4 x^{2}\right) \log x\right],
\end{aligned}
$$

with $x=M_{W}^{2} / M_{H}^{2}$. Using the two-body-like formula given above and the approximation of ignoring the kinematical effects induced by the three-body final-state phase space, we have implemented this additional channel in a DM FORTRAN code based on ref. [40] which is linked to the program SuSpect. Using this routine we have scanned the $\left[M_{2}, \mu\right]$ parameter space and determined the regions in which the WMAP constraint is fulfilled 1 . This is performed not only in the scenario with a universal gaugino mass $m_{1 / 2}$ at the GUT scale but also in scenarios with different boundary conditions.

In fig. 9 we display the area in the $\left[M_{2}, \mu\right]$ parameter space in which the WMAP constraint is fulfilled; a common scalar mass $M_{S}=10^{4} \mathrm{GeV}$ is chosen and a universal gaugino mass at $M_{\mathrm{GUT}}$ is assumed; here and in the subsequent discussions we will fix the value of $\tan \beta$ to $\tan \beta=30$. The green (light grey) area in the left and bottom parts of the figure denotes the region excluded by the collider data discussed in the previous subsection. The peak for small $M_{2}$ values, $M_{2} \sim 2 M_{1} \sim M_{H}$, is due to the $s$-channel exchange of the Higgs boson, $\chi_{1}^{0} \chi_{1}^{0} \rightarrow H$. For the mass value obtained here, $M_{H} \sim 130 \mathrm{GeV}$, the Higgs boson mainly decays into $b \bar{b}$ final states while the $H \rightarrow W W^{*}$ channel, which has also been included, has a smaller branching ratio and does not play a leading role. Between the two bands of the peak

\footnotetext{
${ }^{1}$ For some selected points of the parameter space, we have verified that the results that we obtain for $\Omega h^{2}$ using the routine of ref. [40 are in a relatively good agreement with those obtained with the program micrOMEGAs [41.
} 


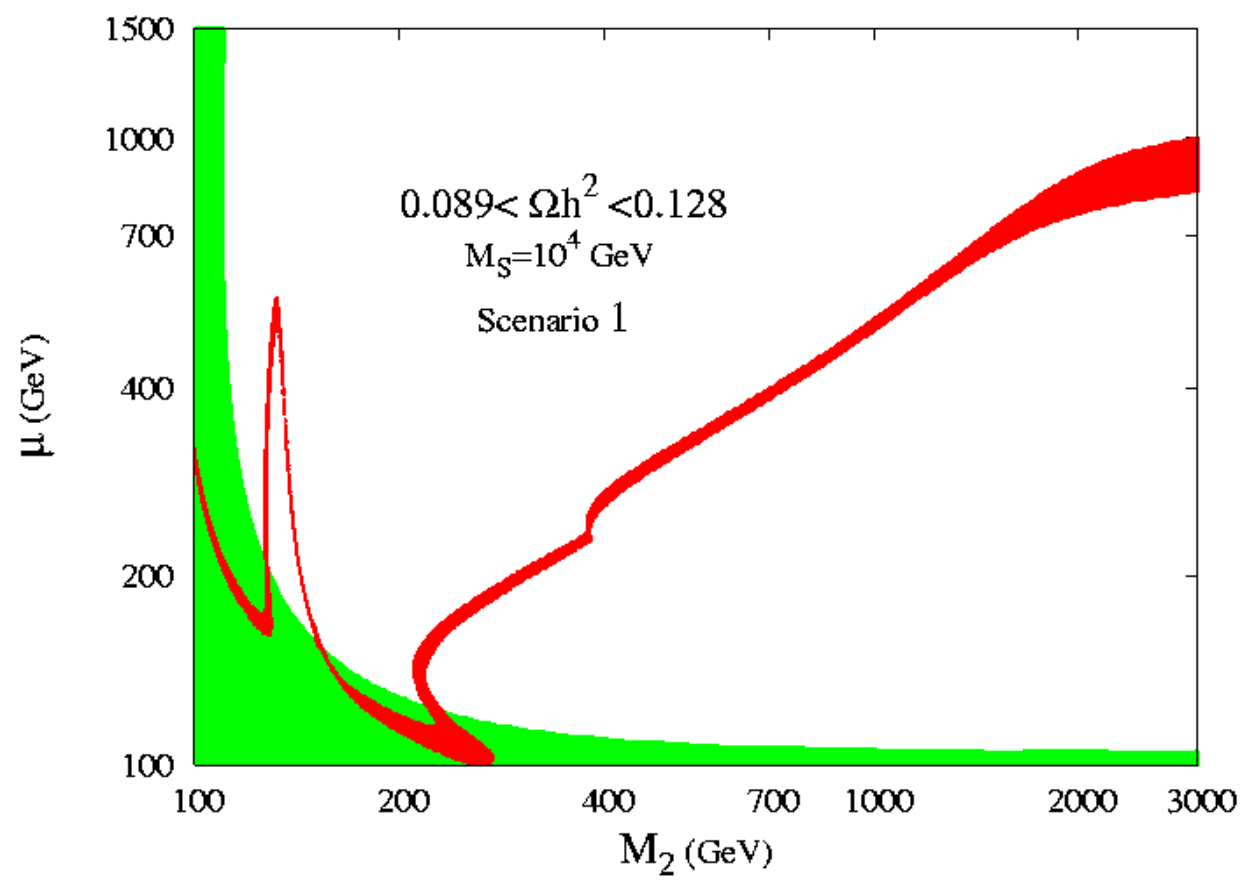

Figure 9: The regions of the $\left[M_{2}, \mu\right]$ parameter space in which the WMAP constraint is fulfilled (red area) for a common scalar mass value $M_{S}=10^{4} \mathrm{GeV}, \tan \beta=30$ and the assumption of a universal gaugino mass at the GUT scale. The green area on the left and the bottom is the one excluded by direct searches of SUSY particles.

one is too close to the Higgs mass pole, and the LSP annihilation is too efficient leading to a too small $\Omega h^{2}$. The peak reaches up to $\mu \sim 600 \mathrm{GeV}$, a value beyond which the LSP is almost bino-like and its coupling to the Higgs boson is too small (the Higgs prefers to couple to a higgsino-gaugino mixture) to generate a sizable annihilation cross section.

For larger $\mu$ and $M_{2}$ values there is an almost straight band in which $\mu \sim M_{1}$ and the LSP is a bino-higgsino mixture with sizable couplings to $W, Z$ and Higgs bosons, allowing for reasonably large rates for neutralino annihilation into $\chi_{1}^{0} \chi_{1}^{0} \rightarrow W^{+} W^{-}, Z Z, H Z$ and $H H$ final states. For instance, for $M_{2} \sim 300 \mathrm{GeV}$ and $\mu=200 \mathrm{GeV}$, the annihilation cross section is mostly due to the $W W$ and $H H$ final states ( $\sim 40 \%$ for both channels) and, to a lesser extent, the $Z Z$ and $Z H$ final states $\left(\sim 10 \%\right.$ each). For slightly larger $\mu$ and $M_{2}$ values there is a jump due to the opening of the $\chi_{1}^{0} \chi_{1}^{0} \rightarrow t \bar{t}$ channel, which then dominates the annihilation cross section. Above the band and below the band, the LSP couplings to the various final states are either too strong or too weak to generate the relevant relic density. For $\mu$ values close to $1 \mathrm{TeV}$ and even larger values of $M_{2}$ there is a wider area in which the WMAP constraint is also fulfilled. In this region the LSP is almost a pure higgsino and a 

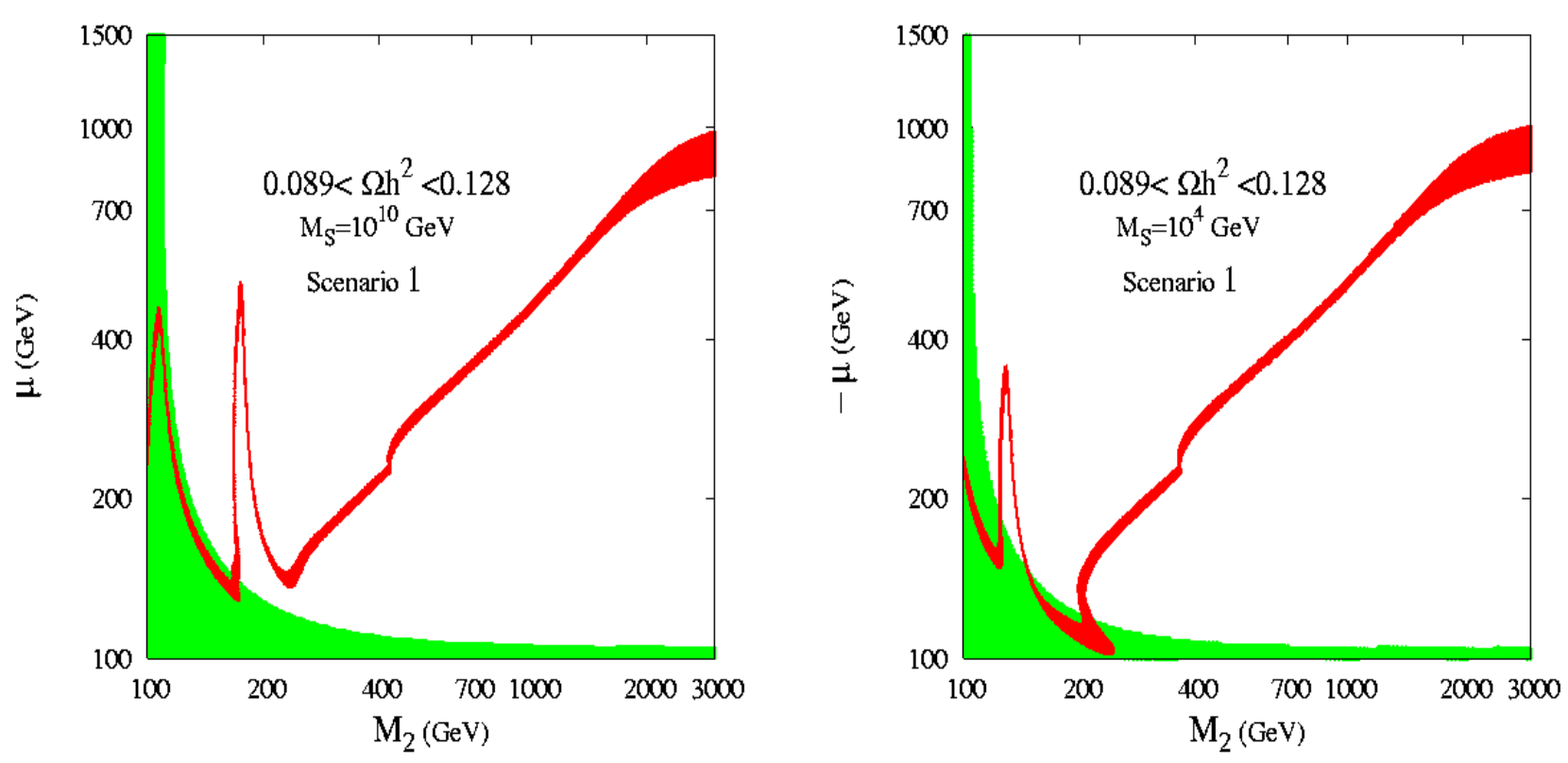

Figure 10: The same as in fig. 9 but with $M_{S}=10^{10} \mathrm{GeV}$ (left) or $\mu<0$ (right)

correct $\Omega h^{2}$ can also be obtained thanks to the co-annihilation of the LSP with the $\chi_{1}^{ \pm}$and $\chi_{2}^{0}$ states. For lower $\mu$ values and $M_{2}$ still very large the LSP co-annihilation with $\chi_{1}^{ \pm}$and $\chi_{2}^{0}$ is too strong and leads to a too small $\Omega h^{2}$.

Figure 10 is similar to the previous one, with the difference that $M_{S}=10^{10} \mathrm{GeV}$ (left pane) or the sign of the parameter $\mu$ is reversed (right pane). The figure in the left-hand side shows similar features as fig. 9, except that the peak due to the $s$-channel Higgs boson exchange is shifted to a slightly higher $M_{2}$ value, $M_{2} \sim 2 M_{1} \sim M_{H} \sim 150 \mathrm{GeV}$. In this case the new annihilation channel $\chi_{1}^{0} \chi_{1}^{0} \rightarrow H \rightarrow W W^{*} \rightarrow W f \bar{f}$ discussed before gives a significant contribution to the total cross-section. For negative $\mu$ values the $\left[M_{2}, \mu\right]$ area of the parameter space that leads to the desired $\Omega h^{2}$ is also similar to fig. 9, The only difference is that the Higgs peak reaches up to $|\mu| \sim 400 \mathrm{GeV}$ only, as for negative $\mu$ values the LSP becomes bino-like more quickly than in the positive case and its couplings to the Higgs boson are thus smaller.

Figure 11 shows the $\left[M_{2}, \mu\right]$ area that is compatible with WMAP results for $M_{S}=10^{4}$ $\mathrm{GeV}$ in the case where the boundary conditions for the gaugino masses at the GUT scale are not universal. In the scenario $\mathbf{2 4}$ the same trend as for the universal case occurs but with two major differences. First, the Higgs peak is now shifted to $M_{2} \sim 400 \mathrm{GeV}$, a mere consequence of the fact that the ratio of the weak-scale wino-to-bino masses is much larger in this model, $M_{2}: M_{1} \sim 6$, than in the universal case, $M_{2}: M_{1} \sim 2$; for the same reason, the band at large $M_{2}$ values is shifted downward compared to fig. 9, The other 
major difference with the universal scenario is that, despite the constraint on the invisible $Z$ decay width $\Gamma\left(Z \rightarrow \chi_{1}^{0} \chi_{1}^{0}\right) \lesssim 2 \mathrm{MeV}$ and the one from chargino and neutralino production at LEP2, the possibility that the LSP mass is close to $\frac{1}{2} M_{Z}$ is not excluded. In this case, the LSP annihilation channel $\chi_{1}^{0} \chi_{1}^{0} \rightarrow Z \rightarrow f \bar{f}$ can become resonant, thus generating the required cosmological relic density. This leads to a peak similar to the one due to Higgs boson exchange but at smaller $M_{2}$ values, $M_{2} \simeq 6 M_{1} \simeq 3 M_{Z} \sim 300 \mathrm{GeV}$.

In the scenario 75 one has $M_{1}: M_{2}: M_{3} \sim 1:-1.2:-1.5$ at the weak scale, so that the LSP is in general close in mass to the lightest chargino and the next-to-lightest neutralino, and co-annihilation of these states plays a very important role. Indeed, in the thin straight line below $\mu, M_{2} \lesssim 1 \mathrm{TeV}$ the LSP is dominantly bino-like (e.g. $\sim 85 \%$ for $\mu=M_{2} \sim 600 \mathrm{GeV}$ ) with a small higgsino component, and the WMAP $\Omega h^{2}$ range is obtained with the efficient co-annihilation of $\chi_{2}^{0} \chi_{1}^{ \pm}, \chi_{1}^{\mp} \chi_{1}^{ \pm}(\sim 50 \%)$ and $\chi_{1}^{0} \chi_{1}^{ \pm}, \chi_{1}^{0} \chi_{2}^{0}(\sim 25 \%)$ with the remaining part due to $\chi_{1}^{0} \chi_{1}^{0}$ annihilation. In the large band with $\mu=900-1100 \mathrm{GeV}$ for $M_{2} \gtrsim 1.2 \mathrm{TeV}$ the LSP is very close to be a pure higgsino state (e.g. $\sim 99 \%$ for $M_{2} \sim 2 \mu \sim 2 \mathrm{TeV}$ ), but the co-annihilation cross sections are still reasonably small, the mass difference between the LSP and the other chargino/neutralino states being large enough. All other areas, including the Higgs peak and the mixed gaugino-higgsino areas that appear in the universal case, are ruled out because of a too efficient co-annihilation of the LSP.

The same situation occurs in the scenario 200, in which the weak-scale gaugino mass parameters are given by $M_{1}: M_{2}: M_{3} \sim 2.4: 1: 1.9$ leading to $\chi_{1}^{0}$ and $\chi_{1}^{ \pm}$states which are wino- or higgsino-like and almost mass-degenerate in most of the parameter space. In this case, co-annihilation with $\chi_{1}^{ \pm}$and $\chi_{2}^{0}$ is too efficient except for the band with $\mu=900-1100$ $\mathrm{GeV}$ and $M_{2} \gtrsim 1.2 \mathrm{TeV}$ as in the scenario 75. In this case the band is extended at larger $\mu$ values by a strip in which the LSP is a wino-higgsino mixture, but, again, the bulk of the relic cosmological density $\Omega h^{2}$ is generated through co-annihilation of the LSP with the lightest chargino $\chi_{1}^{ \pm}$.

Finally, in the case of the OII string model, which leads to a weak-scale gluino mass parameter that is smaller than the wino and bino mass parameters, $M_{1}: M_{2}: M_{3} \sim 1.4: 1.3: 1$ for $M_{S}=10^{4} \mathrm{GeV}$, the gluino is the LSP in a large part of the parameter space and this blue (dark grey) area should therefore be excluded. For $M_{2}$ smaller than $1 \mathrm{TeV}$ the LSP is higgsino-like at low $\mu$ values and co-annihilation of the LSP with $\chi_{1}^{ \pm}$and $\chi_{2}^{0}$ is too efficient, while at high $\mu$ values the LSP is the gluino. Only in a relatively narrow band, $\mu \sim 800-$ $1100 \mathrm{GeV}$, similar to the ones observed in the scenarios $\mathbf{7 5}$ and $\mathbf{2 0 0}$, does one obtain the $\Omega h^{2}$ range required by WMAP, with the LSP being an almost pure higgsino state with a relatively large mass; for instance, for $M_{2} \sim 2.5 \mu \sim 2.5 \mathrm{TeV}$, one has $m_{\chi_{1}^{0}} \sim m_{\chi_{2}^{0}} \sim m_{\chi_{1}^{ \pm}} \sim 1$ $\mathrm{TeV}$ and $\Omega h^{2}$ is almost exclusively generated by co-annihilation (95\%). A very interesting feature occurs at the border between the gluino-LSP area and the band with $\mu \sim 0.8-1 \mathrm{TeV}$ : the main channel that leads to the required range for the relic density is gluino annihilation into gluon and quark pairs, $\tilde{g} \tilde{g} \rightarrow q \bar{q}, g g$ (the co-annihilation $\tilde{g} \tilde{\chi} \rightarrow q \bar{q}$ is suppressed as it is mediated by the super-heavy squarks). For instance, in the point $M_{2} \simeq 1.5 \mathrm{TeV}$ and 

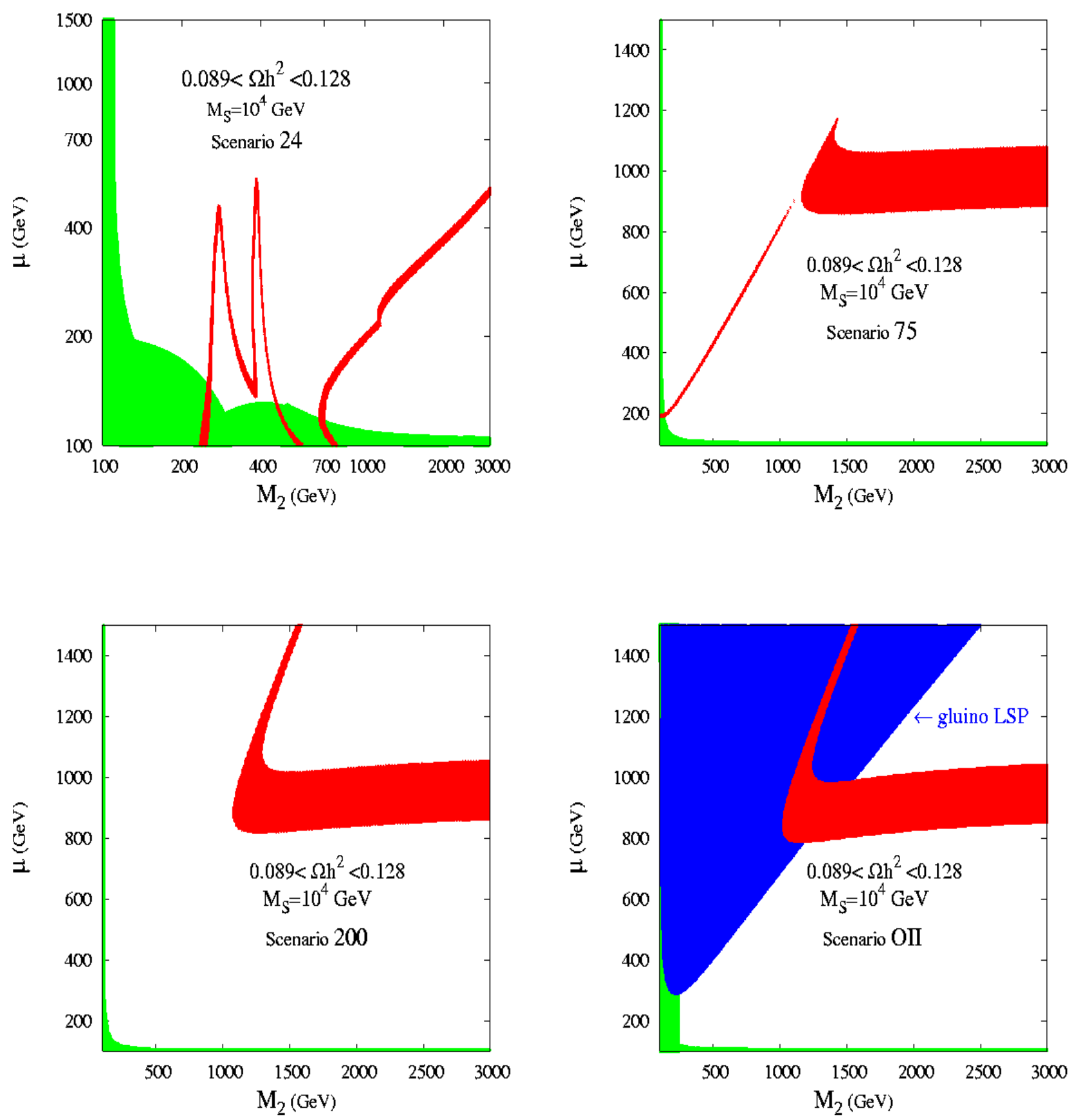

Figure 11: The regions of the $\left[M_{2}, \mu\right]$ parameter space in which the WMAP constraint is fulfilled for $M_{S}=10^{4} \mathrm{GeV}$ in the various models with non-universal GUT-scale boundary conditions for the gaugino mass parameters.

$\mu \simeq 900 \mathrm{GeV}$ one obtains the value $\Omega h^{2} \sim 0.1$ with the $\tilde{g} \tilde{g} \rightarrow g g(45 \%)$ and $\tilde{g} \tilde{g} \rightarrow q \bar{q}(35 \%)$ reactions. This is one of the rare examples within constrained SUSY models where gluino co-annihilation is at work. 


\subsection{The gluino lifetime}

In this section we summarize the constraints that can be obtained on the parameter space, and in particular on $M_{S}$, from the requirement that the gluino lifetime does not exceed the age of the Universe, $\tau_{\tilde{g}} \lesssim 14$ Gyear, a possibility that is excluded from the absence of anomalous isotopes. We extend the discussions of Refs. [32 34], held in the context of universal gaugino masses, to the various scenarios with non-universal boundary conditions at $M_{\mathrm{GUT}}$.

The total decay width of the gluino, $\Gamma_{\tilde{g}}=\hbar / \tau_{\tilde{g}}$, has been calculated following ref. [34], where the large logarithmic corrections that appear for very heavy scalars and are controlled by the strong coupling $\alpha_{s}$ and by the top Yukawa coupling are resummed with an effective Lagrangian approach. Both the three-body decays into charginos or neutralinos and a quarkantiquark pair through the exchange of heavy squarks, $\tilde{g} \rightarrow q \tilde{q}^{*} \rightarrow q \bar{q} \chi_{i}$, and the loop induced decay into a neutralino and a gluon, $\tilde{g} \rightarrow g \chi_{i}^{0}$ have been included (see section 4.3 for more details). The gluino lifetime is approximately given by [34]

$$
\tau_{\tilde{g}}=\frac{\hbar}{\Gamma_{\tilde{g}}}=\frac{4 \mathrm{sec} .}{N}\left(\frac{M_{S}}{10^{9} \mathrm{GeV}}\right)^{4}\left(\frac{1 \mathrm{TeV}}{m_{\tilde{g}}}\right)^{5},
$$

where $N$ is a normalization factor which is generally of order unity if phase-space effects are ignored. This equation exhibits the main trend: the gluino lifetime is larger for higher values of the scalar mass $M_{S}$ and smaller values of the gluino mass $m_{\tilde{g}}$. In Refs. [32 34] it has been shown in the universal scenario that for $M_{S}=\mathcal{O}\left(10^{13}\right) \mathrm{GeV}$ the gluino is almost stable as its lifetime is larger than the age of the Universe.

The gluino lifetime $\tau_{\tilde{g}}$ is displayed in fig. 12 as a function of $M_{S}$ for various values of the gluino mass in the two non-universal scenarios 24 and OII; for each value of $m_{\tilde{g}}$ and hence of $M_{2}$ (the latter obtained via RG evolution from the boundary conditions specific to the scenario) the relevant value of $\mu$ is obtained by requiring that the LSP relic abundance $\Omega_{\chi} h^{2}$ falls in the range allowed by WMAP (see fig. 11). As can be seen, in the scenario 24 a gluino lifetime of the order of the age of the Universe, $\tau_{\tilde{g}}=14$ Gyear (the horizontal line), is obtained for $M_{S}=10^{13}$ to $10^{14} \mathrm{GeV}$ for $m_{\tilde{g}}=1-3 \mathrm{TeV}$. The results are thus similar to the universal scenario discussed in Refs. [8, 34]. In fact, the same results are also obtained in the non universal scenarios 75 and 200 and we refrain from showing them again.

A slightly different situation occurs in the scenario OII, in which the correct DM relic density can be generated when the gluino is close in mass to the higgsino-like neutralino LSP. In this case the only allowed decays of the gluino are the three-body decays into two light quarks and the higgsino-like chargino and neutralinos, and the loop-induced two-body decay into the higgsino-like neutralinos and a gluon (see section 4.3). These decays are suppressed by phase space: $\Gamma \propto\left(m_{\tilde{g}}-m_{\chi}\right)^{5}$ for the three-body decays and $\Gamma \propto\left(m_{\tilde{g}}-m_{\chi}\right)^{3}$ for the radiative decay. Therefore, smaller values of $M_{S}$ are required for the gluino lifetime to be compatible with the age of the Universe. This is exemplified in the right-hand side of fig. 12, 

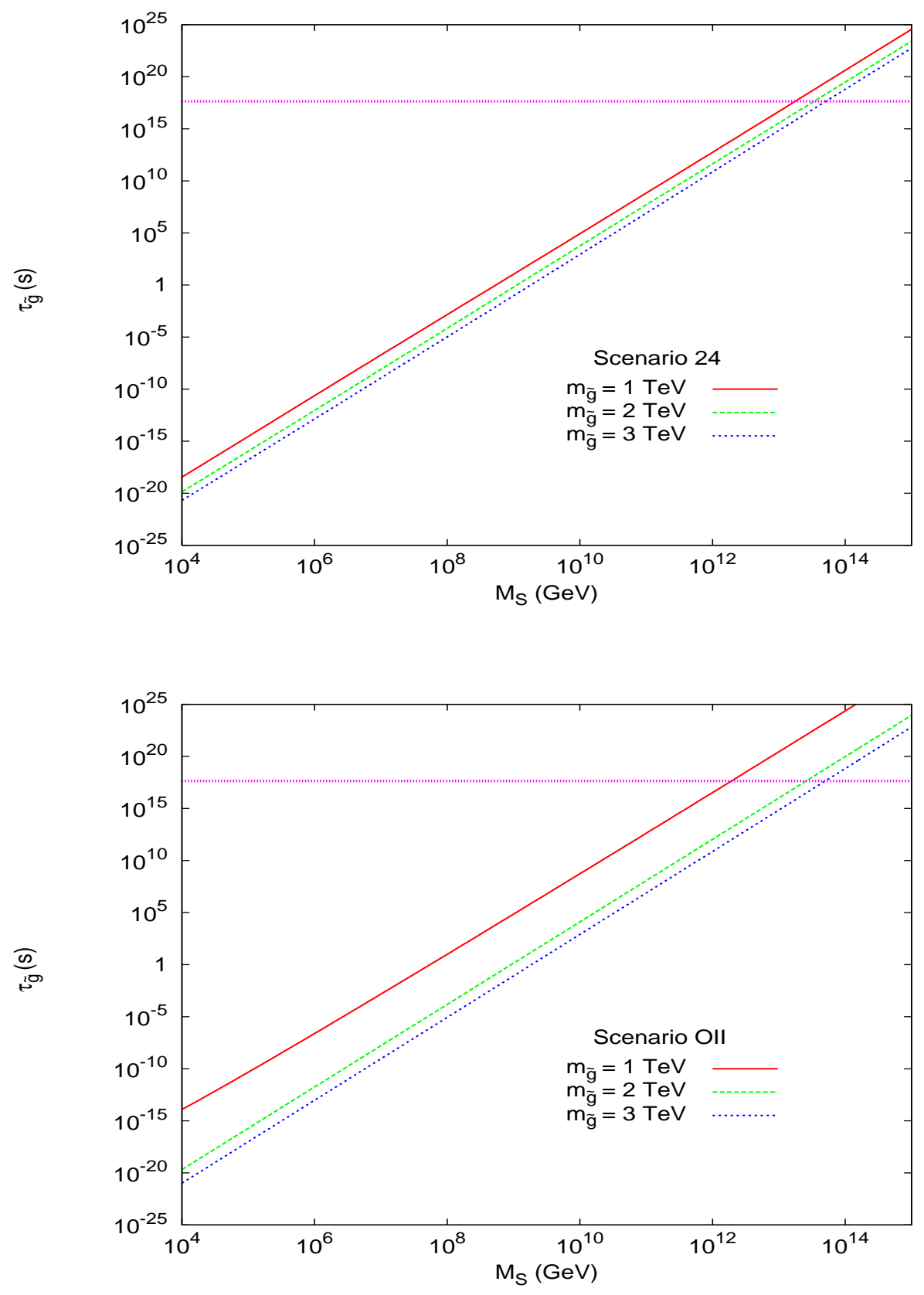

Figure 12: The gluino lifetime (in seconds) as a function of the common scalar mass $M_{S}$ for the two non-universal scenarios 24 and OII for various values of $m_{\tilde{g}}$ and the parameter $\mu$ fixed from the DM requirement; the horizontal line is for the upper bound of 14 Gyear.

where one can see that for $m_{\tilde{g}}=\mathcal{O}\left(1 \mathrm{TeV}\right.$ ) (with $\mu \sim 1 \mathrm{TeV}$ for $\Omega_{\chi} h^{2}$ to fall in the WMAP range) a gluino lifetime of $\tau_{\tilde{g}}=14$ Gyear is already reached for $M_{S} \lesssim 10^{12} \mathrm{GeV}$. 


\section{Decays of the Higgs and SUSY particles}

\subsection{Higgs boson decays}

As discussed in section 2.1, in the MSSM with heavy scalars the Higgs boson is SM-like, except that its mass is constrained to be in the range $M_{H} \sim 130-160 \mathrm{GeV}$ for $M_{S}=10^{4}$ $10^{14} \mathrm{GeV}$ and $\tan \beta=30$ (as will be assumed throughout this section). It will thus decay mostly like the SM Higgs particle in this mass range [26, 42]. For not too large $M_{S}$ values for which $M_{H} \lesssim 130 \mathrm{GeV}$, the Higgs boson decays into a large variety of channels, the main modes being by far the decay into $b \bar{b}$ pairs with a branching ratio of $\sim 90 \%$ followed by the decays into $c \bar{c}$ and $\tau^{+} \tau^{-}$pairs with branching ratios of $\sim 5 \%$. Also of significance, the top-loop mediated Higgs decay into gluons which for $M_{H}$ around $130 \mathrm{GeV}$ occurs at the level of few percent. The top- and $W$-loop mediated $\gamma \gamma$ and $Z \gamma$ decay modes are very rare, with branching ratios of $\mathcal{O}\left(10^{-3}\right)$. However, these decays lead to clear signals and are theoretically interesting, being sensitive to new electrically charged particles such as charginos. For values of $M_{S}$ large enough that $M_{H} \gtrsim 140 \mathrm{GeV}$ the Higgs bosons decay into $W W$ and to a lesser extent $Z Z$ pairs, with one of the gauge bosons being virtual below the threshold. For $M_{S} \gtrsim 10^{10} \mathrm{GeV}$, which leads to $M_{H} \gtrsim 150 \mathrm{GeV}$, the Higgs boson decays almost exclusively into two real $W$ bosons; the decay $H \rightarrow Z Z^{*}$ is strongly suppressed as one of the $Z$ boson must be virtual. In all cases, the Higgs boson is very narrow, as its total decay width does not reach the $1 \mathrm{GeV}$ level.

There are, however, two situations in which the Higgs boson might have decays that are slightly different from those of the SM Higgs particle. First, for very light LSP neutralinos, the invisible decay $H \rightarrow \chi_{1}^{0} \chi_{1}^{0}$ [43] might be kinematically accessible. In the scenario with universal gaugino masses leading to $M_{2} \sim 2 M_{1}$ at the weak scale, this decay occurs for values of $M_{2}$ small enough to have the phase space needed for the decay to occur, $M_{H} \gtrsim 2 m_{\chi_{1}^{0}} \sim$ $2 M_{1}$. In the left-hand side of fig. $\left[13\right.$ the areas in the $\left[M_{2}, \mu\right]$ parameter space in which the branching ratio $\operatorname{BR}\left(H \rightarrow \chi_{1}^{0} \chi_{1}^{0}\right)$ is larger than $1 \%, 5 \%$ and $10 \%$ are shown for $M_{S}=10^{4}$ $\mathrm{GeV}$ and $\tan \beta=30$; the area in which the neutralino relic density is in the range required by WMAP is also displayed, as well as the area excluded by collider bounds. For $M_{2}$ values in the range $120-150 \mathrm{GeV}$ and small $\mu$ values, the branching ratio is of the order of $5 \%$ and sometimes $10 \%$ and is therefore measurable at the ILC. The branching ratio drops with increasing $\mu$ values since for $\mu \gg M_{2}$ the Higgs-LSP coupling becomes too small. In the non-universal scenario 24 with the weak-scale relation $M_{2} \sim 6 M_{1}$ between the wino and bino masses, fig. 13 (right), the corresponding areas in the $\left[M_{2}, \mu\right]$ parameter space are larger as a result of a larger phase space allowed for the invisible Higgs decays. In particular, branching ratios larger than $10 \%$ are possible in a significant portion of the parameter in which the DM constraint is also fulfilled. Thus, if by chance it is the $H$ or $Z$ boson pole which provides the correct value of $\Omega h^{2}$, the invisible decay branching ratio $\operatorname{BR}\left(H \rightarrow \chi_{1}^{0} \chi_{1}^{0}\right)$ could be measured at the ILC and would allow to access directly to the Higgs-LSP couplings. 

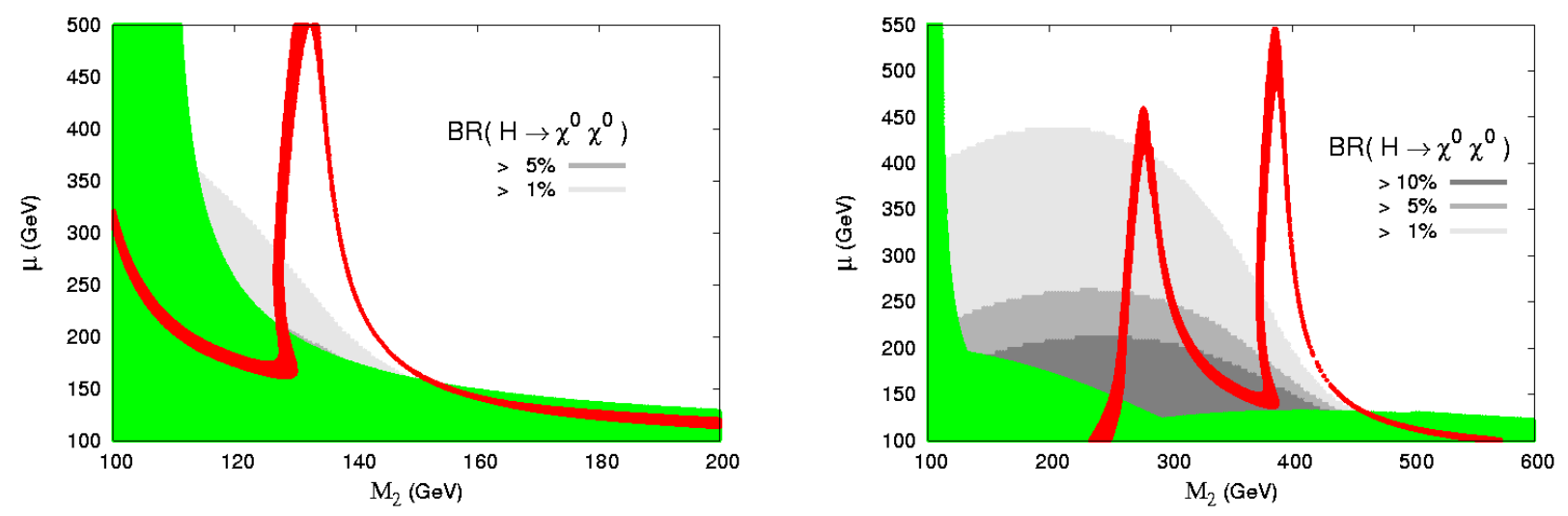

Figure 13: The areas in the $\left[M_{2}, \mu\right]$ plane in which the invisible Higgs branching ratios $\mathrm{BR}\left(H \rightarrow \chi_{1}^{0} \chi_{1}^{0}\right)$ in larger than $1 \%, 5 \%$ and $10 \%$ in the scenarios $\mathbf{1}$ and $\mathbf{2 4}$ with $M_{S}=10^{4}$ $\mathrm{GeV}$ and $\tan \beta=30$; the region in which the relic density is compatible with WMAP is also displayed (red region). The program HDECAY [16], adapted to deal with heavy scalars, has been used.

The decays of the Higgs boson into the heavier neutralinos and the charginos are in general kinematically closed if one takes into account the LEP2 bounds on the masses of these particles. There is however one possible exception: in the scenario $\mathbf{2 4}$, because the absolute lower limit on the LSP mass is only $m_{\tilde{\chi}_{1}^{0}} \gtrsim 17 \mathrm{GeV}$, the possibility of the decay $H \rightarrow \chi_{1}^{0} \chi_{2}^{0}$ is still open. This occurs for values of $M_{1}$ and $M_{2}$ that lie very close to those ruled out by the experimental constraints and, for such values, the requirement that the LSP provides the correct relic density is not fulfilled.

Another possibility for a deviation from the SM predictions for the Higgs decays is when the charginos are light enough to contribute to the loop decay $H \rightarrow \gamma \gamma$ (contributions to the $H \rightarrow Z \gamma$ decays are in general much smaller) [44. Indeed, besides top quark and $W$ boson loops, one has to include also the contribution of the chargino loops. However, in contrast to the SM particles, the charginos do not couple to the Higgs boson proportionally to their masses and the amplitudes are damped by inverse powers of the $\chi^{ \pm}$masses. The chargino contributions are thus sizable only for relatively small masses; see also ref. [45] in which this topic has been discussed in the Split-SUSY scenario.

This is exemplified in fig. 14, where the regions in the $\left[M_{2}, \mu\right]$ plane in which the deviation of $\Gamma(H \rightarrow \gamma \gamma)$ from SM prediction is larger than $1 \%$ and $2 \%$ are displayed in the universal gaugino mass scenario with $M_{S}=10^{4} \mathrm{GeV}$; the area where the WMAP DM constraint in fulfilled has been superimposed. Only for small $M_{2}$ values, and thus rather light chargino states, does the deviation from the SM prediction exceed the level of $2 \%$, which makes it potentially observable at the $\gamma \gamma$ option of the ILC (where one expects the $H \gamma \gamma$ coupling to be measured at the two-percent level). The sign of the contribution is controlled by the sign of 


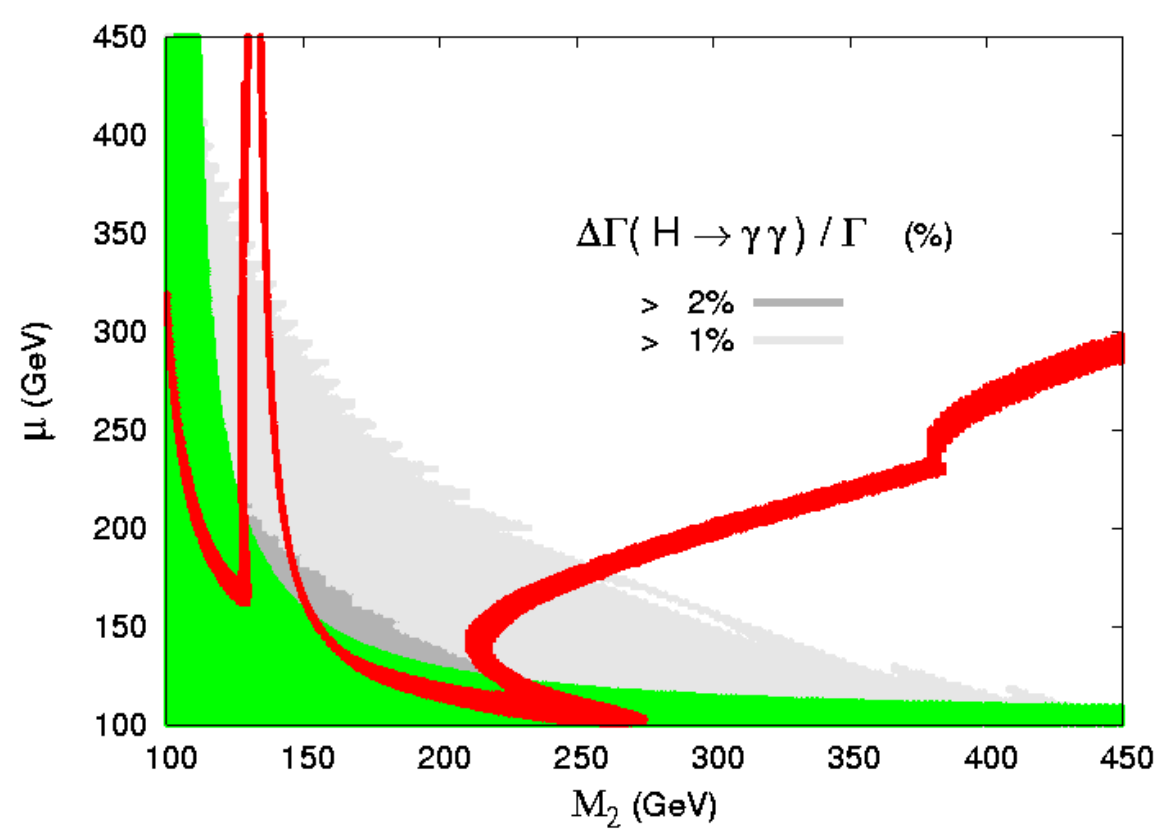

Figure 14: The areas of the $\left[M_{2}, \mu\right]$ plane in which the deviation of $\Gamma(H \rightarrow \gamma \gamma)$ from the SM prediction is larger than $1 \%$ and $2 \%$ for $M_{S}=10^{4} \mathrm{GeV}$; the area where the WMAP DM constraint in fulfilled is also shown. The program HDECAY [16], adapted to deal with heavy scalars, has been used.

$\mu$, thus for $\mu<0$ one would have a negative shift in $\Gamma(H \rightarrow \gamma \gamma)$. The chargino contribution drops for higher $\mu$ values as, in this case, the chargino $\chi_{1}^{ \pm}$which gives the most important contribution becomes more wino-like and has a weaker coupling to the Higgs boson.

Since the chargino masses depend only on $M_{2}$ and $\mu$, the same figure holds for the nonuniversal scenarios; the only difference is that the WMAP-allowed areas for the LSP relic density, which have been given in fig. 11, would be different. In fact, one can see from these figures that in the scenarios 75, 200 and OII the DM constraint is fulfilled only for large $\mu, M_{2}$ values, thus for charginos too heavy to contribute to the $\gamma \gamma$ decay of the Higgs boson. Furthermore, in these scenarios neutralino LSPs that are compatible with WMAP data are in general also too heavy to allow for the occurrence of the $H \rightarrow \chi_{1}^{0} \chi_{1}^{0}$ invisible decays discussed earlier.

Note that for very large $M_{S}$ values, $M_{S} \gtrsim 10^{10} \mathrm{GeV}$ when the Higgs mass $M_{H} \sim 160$ $\mathrm{GeV}$ becomes close to the $W W$ threshold, the branching ratios for both the invisible decay and the $\gamma \gamma$ decay of the Higgs boson become smaller than for $M_{S}=10^{4} \mathrm{GeV}$, as these decays have to compete with the $H \rightarrow W W^{*}$ channel, which has a much larger decay rate than the $H \rightarrow b \bar{b}$ channel. 


\subsection{Chargino and neutralino decays}

In most cases, the charginos and the neutralinos (except for the LSP) will decay into lighter $\chi$ states and $V=W / Z$ gauge bosons which can possibly be virtual and subsequently decay into two massless fermions, $\chi_{i} \rightarrow \chi_{j} V^{(*)} \rightarrow \chi_{i} f \bar{f}$ [46]. As the scalar fermions are very heavy, their virtual exchange $\chi_{i} \rightarrow f \tilde{f}^{*} \rightarrow f \bar{f} \chi_{j}$ is strongly suppressed and they do not participate in the decay processes. The branching ratio for the full final states will thus essentially follow that of the gauge bosons, therefore the branching ratios into $\ell=\mu, \nu$ leptons are rather small: $20 \%$ for the charged and $6 \%$ for the neutral decays. There are however two interesting features which might occur and which will be briefly discussed below: $i$ ) decays of the heavier charginos/neutralinos into lighter ones and the Higgs boson, $\chi_{i} \rightarrow \chi_{j} H$ [4] and ii) the loop-induced decay of some neutralinos into the LSP and a photon, $\chi_{i}^{0} \rightarrow \chi_{1}^{0} \gamma$ [48]. The former process would allow to access directly the $H \chi \chi$ couplings and the latter has an interesting experimental signature, a monochromatic photon.

Adapting the program SDECAY [17] to the case of heavy scalars, we have calculated the branching ratios for the decays of the charginos and heavier neutralinos into lighter states and the Higgs boson. The result is illustrated in fig. 15, where the branching ratios $\operatorname{BR}\left(\chi_{i} \rightarrow \chi_{j} H\right)$ are shown as a function of $\mu$ in the two scenarios $\mathbf{1}$ (top) and $\mathbf{2 4}$ (bottom) for $M_{S}=10^{4} \mathrm{GeV}$; for a given $\mu$, the value of $M_{2}$ is chosen in such a way that we sit on the left band of the Higgs peak in the WMAP-allowed region of the $\left[M_{2}, \mu\right]$ plane (see figs. 9 and 11). Two main ingredients control the size of the $\chi_{i} \rightarrow \chi_{j} H$ branching fractions:

i) The mass difference between the initial and final $\chi$ states and hence the importance of the phase space; in fact, the $\chi_{i}-\chi_{j}$ mass difference needs to be larger than $M_{H}$ for the Higgs boson to be on-shell, as the $H^{*} \rightarrow f \bar{f}$ virtuality would be strongly suppressed by the small $H f \bar{f}$ couplings.

ii) The initial and final neutralino and chargino states should have different textures as to maximize the $H \chi_{i} \chi_{j}$ coupling.

As can be seen from the figures, some decays such as $\chi_{2}^{ \pm} \rightarrow \chi_{1}^{ \pm} H$ and $\chi_{4}^{0} \rightarrow \chi_{2}^{0} H$ can reach the $20 \%$ level. In the scenario 1 the other decay modes involving the Higgs boson are below the $10 \%$ level as they are suppressed either by phase space or by the smaller $H \chi \chi$ couplings. In the scenario $\mathbf{2 4}$ an interesting feature occurs for intermediate $\mu$ values, $\mu=300-450 \mathrm{GeV}$, where all charginos and neutralinos are mixed gaugino-higgsino states and have masses of the same order. In this range, the dominant decay channels mentioned above are kinematically closed, allowing for the phase-space favored decays $\chi_{3,4}^{0} \rightarrow \chi_{1}^{0} H$ to dominate.

For the radiative and loop-induced decay $\chi_{i}^{0} \rightarrow \chi_{j}^{0} \gamma$ (which is of higher order in perturbation theory and thus suppressed by additional powers of the electroweak coupling) to occur with a substantial rate, the standard decay modes $\chi_{i}^{0} \rightarrow \chi_{j}^{0} Z, \chi_{j}^{0} H$ need to be strongly suppressed. This occurs when the $\chi_{i}^{0}-\chi_{j}^{0}$ mass difference is smaller than $M_{Z}$ and thus $M_{H}$, so that the decay is a three-body process with a partial width that is suppressed by the 

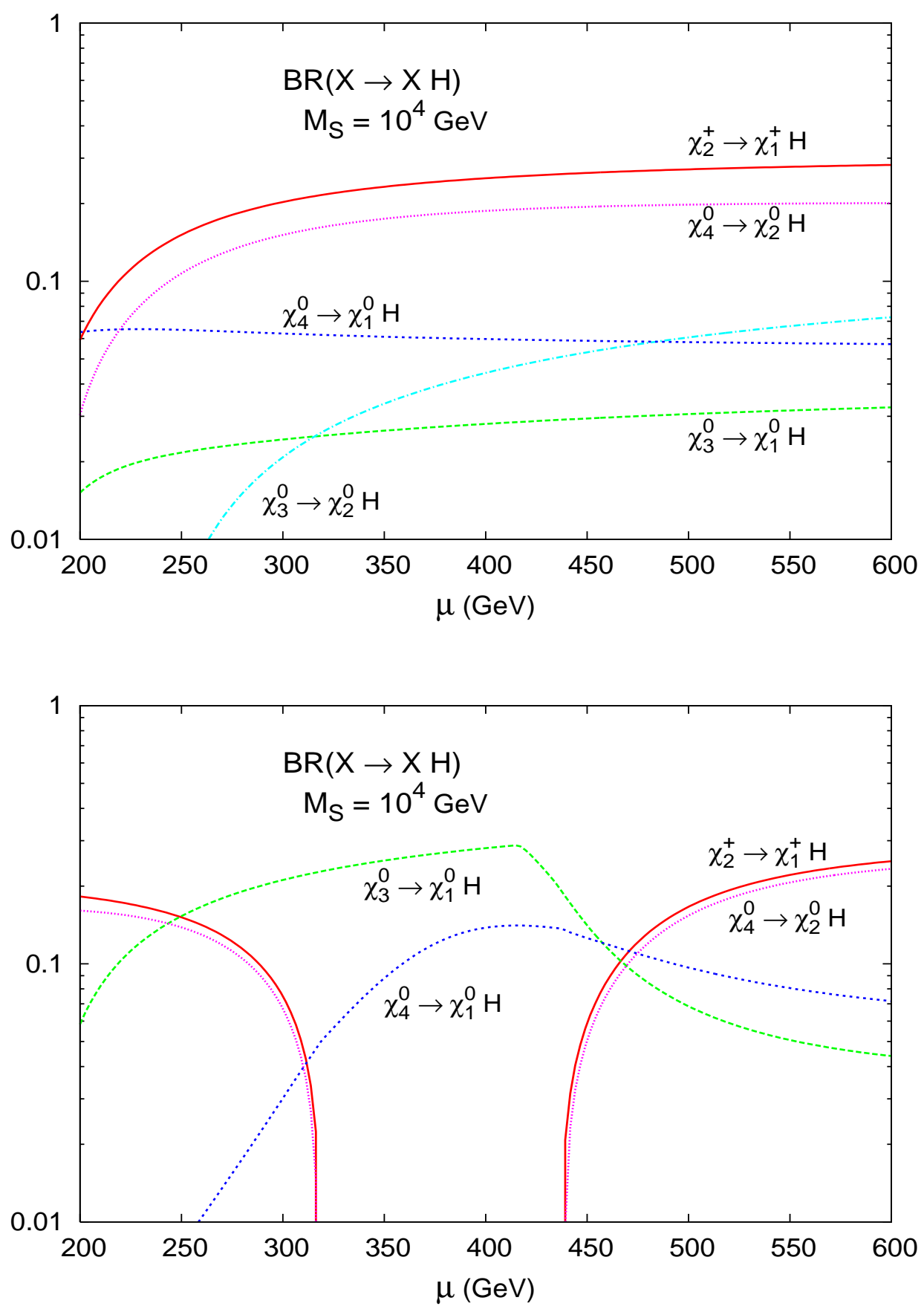

Figure 15: The branching ratios for the decays of the heavier charginos and neutralinos into lighter ones and Higgs bosons, $\operatorname{BR}\left(\chi_{i} \rightarrow \chi_{j} H\right)$ as a function of $\mu$ in the scenarios 1 (top) and 24 (bottom); $M_{S}=10^{4} \mathrm{GeV}$ is assumed and $M_{2}$ is chosen such that the relic density is compatible with WMAP. The program SDECAY [17, adapted to deal with heavy scalars, has been used. 


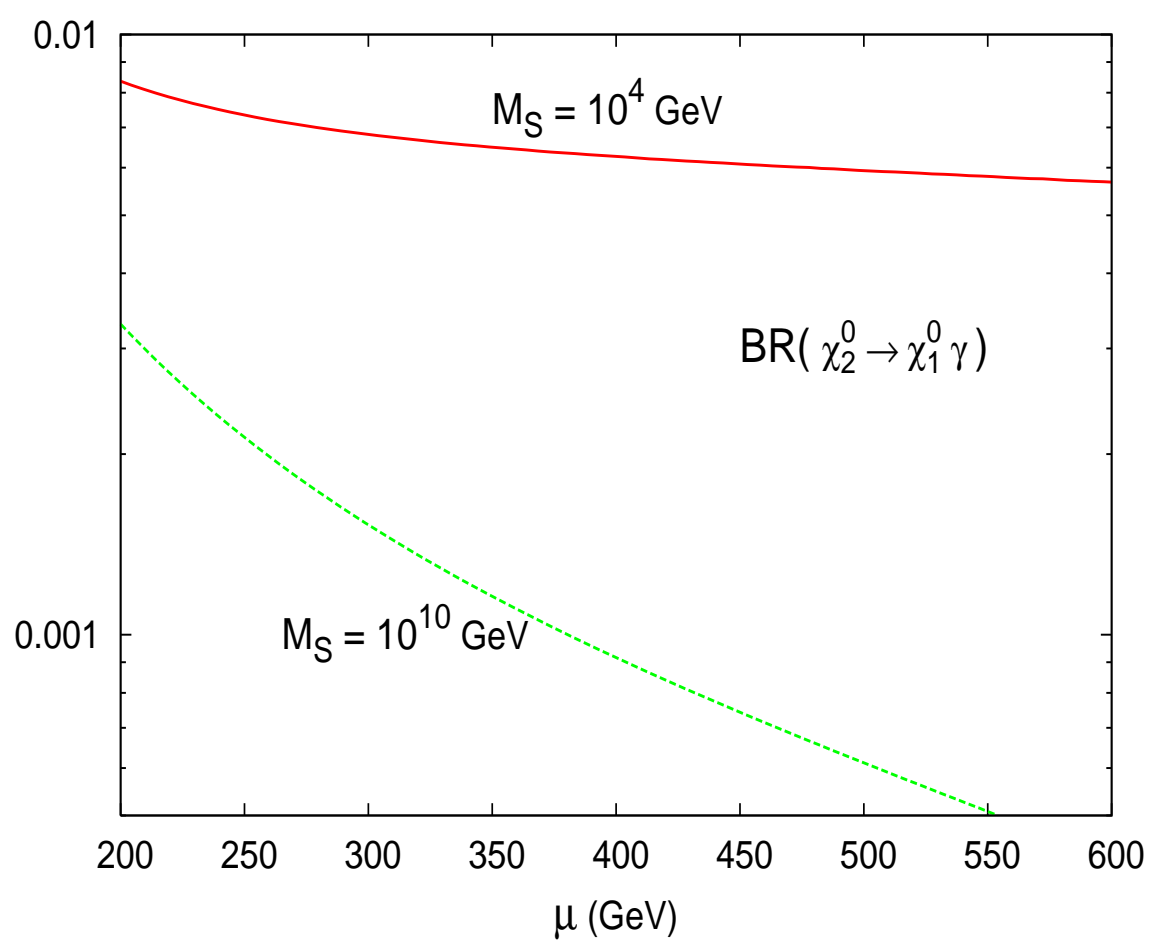

Figure 16: The branching ratios for the radiative decay $\chi_{2}^{0} \rightarrow \chi_{1}^{0} \gamma$ as a function of $\mu$ in the scenario 1 with $M_{S}=10^{4} \mathrm{GeV}$ and $10^{10} \mathrm{GeV}$, and $M_{2}$ chosen such that the relic density is compatible with WMAP. The program SDECAY [17] has been used.

virtuality of the $Z$ boson and by the additional $Z f \bar{f}$ coupling. Furthermore, the $Z \chi_{i}^{0} \chi_{j}^{0}$ coupling needs to be strongly suppressed, thus the initial and final neutralinos need to be either pure gauginos or pure higgsinos. However, as discussed in section 3.2, for the LSP to form the DM in the Universe in such conditions its mass should be in the $\mathrm{TeV}$ range, see figs. 910. Therefore, for relatively light neutralinos that lead to the cosmological relic density favored by WMAP (and, hence, have at least a small higgsino component leading to a non-negligible coupling to the $Z$ boson), the branching ratio $\operatorname{BR}\left(\chi_{i}^{0} \rightarrow \chi_{1}^{0} \gamma\right)$ is expected to be very small.

This is exemplified in fig. 16 where the branching ratio for the decay of the next-tolightest neutralino into the LSP and a photon, $\operatorname{BR}\left(\chi_{2}^{0} \rightarrow \chi_{1}^{0} \gamma\right)$, is displayed in the universal scenario 1 as a function of $\mu$ for $M_{S}=10^{4} \mathrm{GeV}$ and $10^{10} \mathrm{GeV}$; as usual, the value of $M_{2}$ is adjusted in such a way that the WMAP DM constraint is fulfilled. As can be seen, the branching ratio hardly reaches the level of $1 \%$ for $M_{S}=10^{4} \mathrm{GeV}$. For $M_{S}=10^{10} \mathrm{GeV}$, the branching fraction is even smaller as the splitting $M_{2}-M_{1}$ is larger, see table 1 , leading to a more favored phase space for $\chi_{2}^{0} \rightarrow \chi_{1}^{0} Z^{*}$ which controls the total decay width. 


\subsection{Gluino decays}

As already discussed in section 3.3, the gluinos decay through virtual heavy squark exchange either into neutralinos or charginos and a quark-antiquark pair [46], $\tilde{g} \rightarrow \chi_{i}^{0} q \bar{q}$ or $\tilde{g} \rightarrow \chi_{i}^{ \pm} q \bar{q}^{\prime}$, or into the two-body neutralino-gluon final state [49], $\tilde{g} \rightarrow \chi_{i}^{0} g$, which is mediated by loops involving quarks and heavy squarks. Thus, the final state topology will consist of a neutralino or a chargino (which, if it is not the LSP neutralino, will subsequently decay according to the discussion held in the previous subsection) and one or two hard jets.

It is experimentally important to know the number of final-state jets and, thus, the relative magnitude of the branching fractions for the-loop induced decays and the tree-level three-body decays. We have thus scanned the usual $\left[M_{2}, \mu\right]$ parameter space and delineated the areas in which the branching ratio $\operatorname{BR}\left(\tilde{g} \rightarrow g \sum_{i} \chi_{i}^{0}\right)$, summed over all four neutralinos, is larger than 1,5,10,25 and 50\% (from lighter colors to darker ones). The results for the universal gaugino mass scenario are shown in fig. 17 for a common scalar mass values of $M_{S}=10^{4} \mathrm{GeV}$ (left) and $M_{S}=10^{10} \mathrm{GeV}$ (right); as usual the areas in which the WMAP constraint is satisfied and those excluded by the LEP bounds are also displayed.

As can be seen, the branching ratio $\operatorname{BR}\left(\tilde{g} \rightarrow g \sum_{i} \chi_{i}^{0}\right)$ is larger for lighter gluinos and thus smaller values of $M_{2}$, and for larger values of the scalar quark mass $M_{S}$. For instance, the $g \sum_{i} \chi_{i}^{0}$ branching ratio exceeds the level of $50 \%$ for $M_{2} \lesssim 200 \mathrm{GeV}$ and $M_{S}=10^{10} \mathrm{GeV}$. As discussed in Refs. [8, 34], this is due to the fact that the main contribution to the radiative decay originates from loops involving top quarks and squarks (which have large couplings, $\propto m_{t}$, to the higgsino components) and the ratio between this decay and the three-body decay scales as

$$
m_{t}^{2} / m_{\tilde{g}}^{2}\left[1-\log \left(M_{S}^{2} / m_{t}^{2}\right)\right]^{2} .
$$

In particular, the branching ratio is substantial in the region where the cosmological relic density is generated by LSP annihilation through Higgs boson exchange, in which all neutralinos are relatively light and thus kinematically accessible.

Similar results are obtained in the case where SUSY breaking occurs through an $F$-term that is not an $\mathrm{SU}(5)$ singlet. As an illustration, the areas of the $\left[M_{2}, \mu\right]$ plane in which $\operatorname{BR}\left(\tilde{g} \rightarrow g \sum_{i} \chi_{i}^{0}\right)$ is larger than 1,5,10,25 and 50\% are shown in the left-hand side of fig. 18 for the scenario 24 with $M_{S}=10^{4} \mathrm{GeV}$. As can be seen, for the same value of $M_{2}$ the branching ratio is significantly larger than in the scenario $\mathbf{1}$. For $M_{S}=10^{10} \mathrm{GeV}$, which is not shown, the decay $\tilde{g} \rightarrow g \sum_{i} \chi_{i}^{0}$ is by far dominating compared to the three-body decay for low $M_{2}$ values. The branching ratio for the string-inspired OII model is shown in the right-hand side of fig. 18, In this case the radiative $\tilde{g} \rightarrow g \sum_{i} \chi_{i}^{0}$ decay is dominant in a large band in which the mass of the gluino is close to that of the LSP neutralino, even for $M_{S}=10^{4} \mathrm{GeV}$. This band intersects the one in which the LSP has the correct density. Thus, there are sizable areas of the parameter space in which the radiative gluino decay is significant and even dominant. 

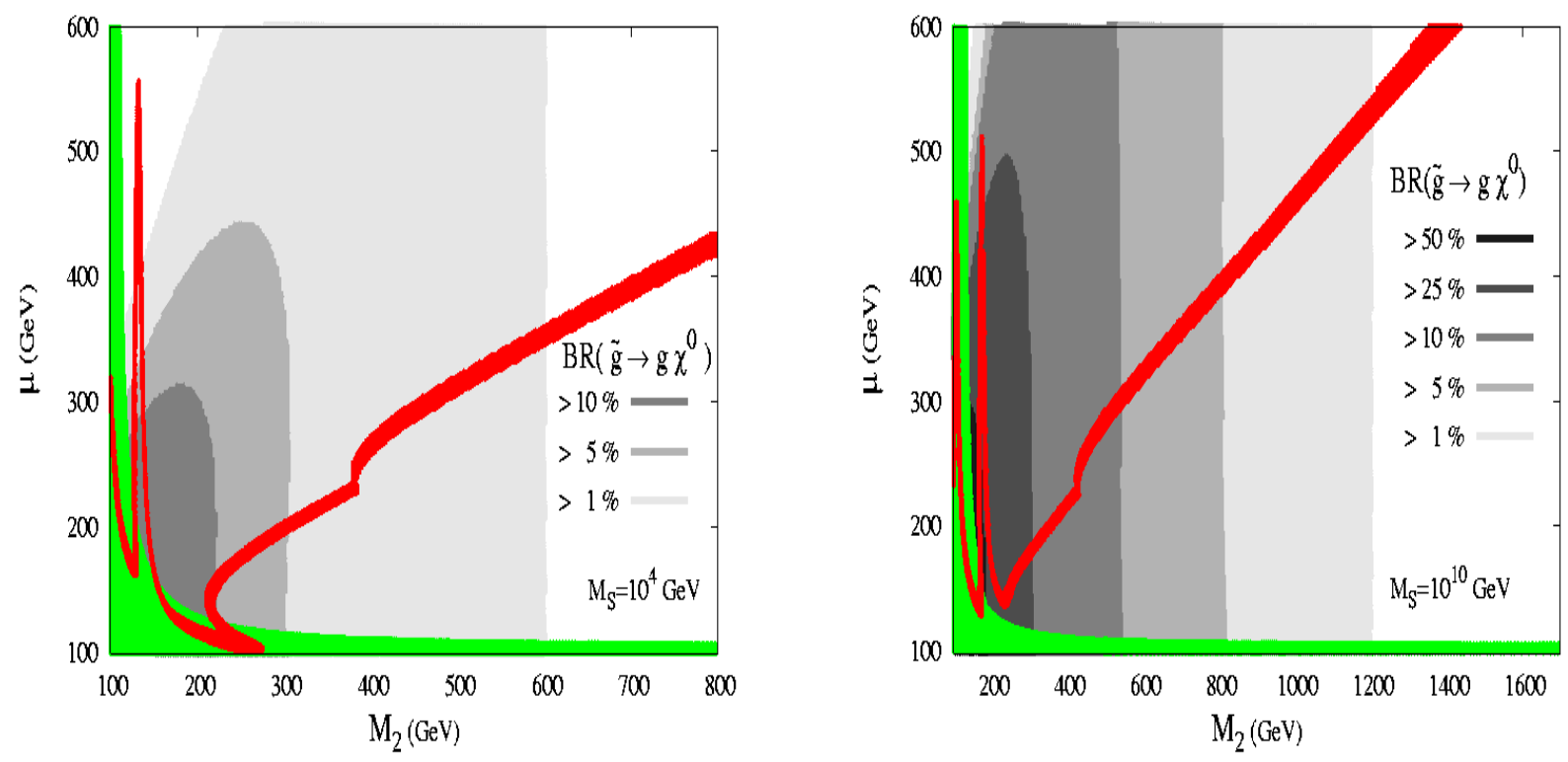

Figure 17: The branching ratios for the decays of gluinos into neutralinos and gluons, $\operatorname{BR}\left(\tilde{g} \rightarrow g \sum_{i} \chi_{i}^{0}\right)$ in the $\left[M_{2}, \mu\right]$ plane in the scenario 1 with $M_{S}=10^{4} \mathrm{GeV}$ (left) and $10^{10} \mathrm{GeV}$ (right); the region in which $\Omega h^{2}$ is compatible with WMAP is also shown.
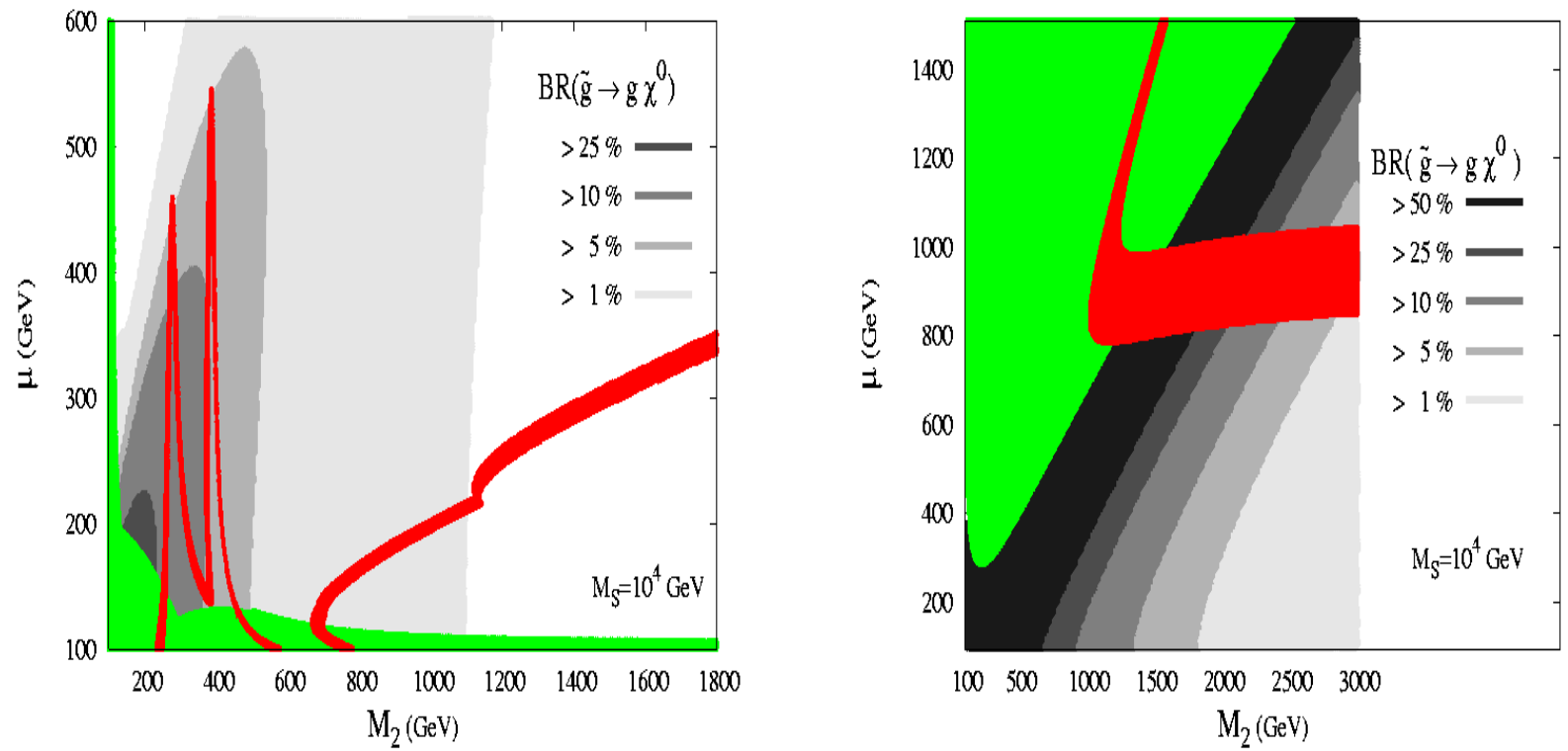

Figure 18: The same as in fig. 17 for the scenarios 24 (left) and OII (right) with $M_{S}=$ $10^{4} \mathrm{GeV}$. 


\section{Conclusions}

We have performed a comprehensive analysis of the MSSM in the scenario where all the scalars, except for the SM-like Higgs boson, are very heavy. This model, commonly known as Split Supersymmetry, is phenomenologically viable and much more predictive than the usual MSSM with light scalars. Indeed, besides the three soft-SUSY breaking mass parameters for the bino, wino and gluino, one has as basic inputs only the common scalar soft-SUSY breaking mass parameter $M_{S}$, the higgsino mass parameter $\mu$, which is not fixed by the requirement of proper radiative electroweak symmetry breaking, and the parameter $\tan \beta$. The model retains the interesting features of the usual MSSM, that is, it leads to a consistent unification of the gauge coupling constants at the GUT scale and provides a solution to the Dark Matter problem in the Universe. Nevertheless, as the scalars are extremely heavy, a large amount of fine tuning in the Higgs sector is present.

In a first step, we presented the model and summarized our precise determination of the masses of the SM-like Higgs boson, the charginos, neutralinos and gluino, and of the different couplings of these particles. In our computation we resum large logarithmic corrections by means of the appropriate RG evolution, and we also include finite one-loop corrections to the particle masses. We performed an analysis of the variation of these masses with respect to the renormalization scale, which can be viewed as as rough estimate of the higher-order corrections. We then discussed the boundary conditions for the soft SUSY-breaking gaugino mass parameters and considered, besides the universal scenario in which they are set to a common value at the GUT scale, two representative sets of scenarios in which the GUT values are non-universal: one where SUSY breaking occurs via an F-term that is not an SU(5) singlet and another based on an orbifold string model. Finally, we briefly described how this model is implemented in the RGE Fortran code SuSpect which calculates the SUSY and Higgs particle spectra in the MSSM.

In a second step, we summarized the available constraints on the model, first from collider searches and high-precision measurements and then from the requirement that the cosmological relic density of the lightest neutralino, which is expected to form the Dark Matter in our Universe, is compatible with the measurements made by the WMAP satellite. These analyses have been performed in the cases of universal and non-universal gaugino mass parameters. It turns out that several new features appear in the non-universal scenarios: in the case of the DM constraint, new channels such as neutralino annihilation through the exchange of a $Z$ boson (which is ruled out by collider constraints in the case of universal gaugino masses) and gluino co-annihilation (which does not occur in the universal scenario) are possible. Furthermore, the annihilation of neutralinos through the exchange of a Higgs boson which then

decays into a real and virtual $W$ boson, leading to the three-body $H \rightarrow W f \bar{f}$ final state, has to be taken into account. We also discussed the constraints on the common scalar mass parameter $M_{S}$ from the requirement that the gluino lifetime does not exceed the age of the Universe, and showed that there are small differences in the non-universal cases compared 
to the case of a universal gaugino mass parameter.

We finally analyzed the phenomenology of the model, focusing on the decays of the Higgs boson and those of the charginos, neutralinos and gluinos. We emphasized the differences between the universal and non-universal cases and, for the Higgs boson decays, the differences between the SM and SUSY cases. For instance, we have shown that the invisible Higgs-boson decay $H \rightarrow \chi_{1}^{0} \chi_{1}^{0}$ can be substantial, reaching branching ratios of the order of $10 \%$ in models where the bino mass parameter $M_{1}$ is much smaller that the wino mass parameter $M_{2}$. The virtual contributions of charginos to the two-photon decay of the Higgs boson have also been shown to reach the level where they can be observable at the $\gamma \gamma$ option of the future linear $e^{+} e^{-}$collider. Decays of heavier charginos and neutralinos into lighter ones and a Higgs boson have been shown to be potentially large, opening the possibility of measuring the $H \chi \chi$ couplings, while the loop-induced decays of heavier neutralinos into lighter ones and a photon are very rare, barely reaching the $1 \%$ level. Finally, we have compared the three-body decays of gluinos through the virtual exchange of heavy squarks, $\tilde{g} \rightarrow q \bar{q} \chi$, with the loop-induced two-body decays $\tilde{g} \rightarrow g \chi$, and have shown the latter to be potentially dominant in some non-universal scenarios.

\section{Acknowledgments:}

We thank Manuel Drees for very useful discussions on the DM code of ref. [40]. This work is supported by the French ANR project PHYS@COS\&COL. 


\section{Appendix}

In this appendix we present for completeness all the formulae for the one-loop radiative corrections that we include in the computation of the mass spectrum of the low-energy effective theory of Split SUSY. We largely follow the notation and the results of ref. [14], adapting when necessary the formulae of that paper to the case of Split SUSY.

\section{Gauge and Yukawa couplings}

We start by summarizing how the minimally renormalized gauge and third-family-Yukawa couplings, as well as the electroweak parameter $\hat{v}$ (from now on a hat denotes minimally renormalized parameters of the Split-SUSY effective theory), are extracted at the scale $Q=$ $M_{Z}$ from the set of SM input parameters $\left[\alpha_{s}\left(M_{Z}\right), \alpha\left(M_{Z}\right), M_{Z}, G_{F}, M_{t}, M_{\tau}, m_{b}\left(m_{b}\right)\right]$. The strong gauge coupling $\hat{\alpha}_{s}$ is extracted from the SM input $\alpha_{s}\left(M_{Z}\right)$ as

$$
\hat{\alpha}_{s}\left(M_{Z}\right)=\frac{\alpha_{s}\left(M_{Z}\right)}{1-\Delta \alpha_{s}}, \quad \Delta \alpha_{s}=\frac{\alpha_{s}}{2 \pi}\left[\delta_{s}-\frac{2}{3} \log \frac{M_{t}}{M_{Z}}-2 \log \frac{M_{\tilde{g}}}{M_{Z}}\right],
$$

where $\delta_{s}$ is a conversion factor that depends on the choice of the minimal renormalization scheme, i.e. $\delta_{s}=0$ in $\overline{\mathrm{MS}}$ and $\delta_{s}=1 / 2$ in $\overline{\mathrm{DR}}$. Similarly, the electromagnetic coupling $\hat{\alpha}$ is extracted from the SM input $\alpha\left(M_{Z}\right)$

$$
\hat{\alpha}\left(M_{Z}\right)=\frac{\alpha\left(M_{Z}\right)}{1-\Delta \alpha}, \quad \Delta \alpha=\frac{\alpha}{2 \pi}\left[\delta_{e}-\frac{16}{9} \log \frac{M_{t}}{M_{Z}}-\frac{4}{3} \sum_{i=1}^{2} \log \frac{\left|m_{\chi_{i}^{+}}\right|}{M_{Z}}\right],
$$

where $\delta_{e}=0$ in $\overline{\mathrm{MS}}$ and $\delta_{e}=1 / 3$ in $\overline{\mathrm{DR}}$. The weak mixing angle (we denote $\sin \theta_{W}$ and $\cos \theta_{W}$ as $s$ and $c$, respectively; we thus define $\hat{s}^{2}=\hat{g}^{\prime 2} /\left(\hat{g}^{2}+\hat{g}^{\prime 2}\right)$ and $\left.s^{2}=1-M_{W}^{2} / M_{Z}^{2}\right)$ is computed from

$$
\hat{c}^{2} \hat{s}^{2}=\frac{\pi \hat{\alpha}}{\sqrt{2} M_{Z}^{2} G_{F}(1-\Delta r)}, \quad \Delta r=\hat{\rho} \frac{\Pi_{W W}(0)}{M_{W}^{2}}-\frac{\Pi_{Z Z}\left(M_{Z}^{2}\right)}{M_{Z}^{2}}+\delta_{V B},
$$

where $\Pi_{W W}\left(p^{2}\right)$ and $\Pi_{Z Z}\left(p^{2}\right)$ are the transverse (and real) part of the $W$ and $Z$ self-energy, respectively, and will be explicitly given below. The $\hat{\rho}$ parameter is defined as

$$
\hat{\rho}=\frac{1+\Pi_{Z Z}\left(M_{Z}^{2}\right) / M_{Z}^{2}}{1+\Pi_{W W}\left(M_{W}^{2}\right) / M_{W}^{2}},
$$

while the quantity $\delta_{V B}$, which accounts for the vertex and box corrections to the muon decay, is:

$$
\delta_{V B}=\hat{\rho} \frac{\hat{\alpha}}{4 \pi \hat{s}^{2}}\left\{6+\frac{\log c^{2}}{s^{2}}\left[\frac{7}{2}-\frac{5}{2} s^{2}-\hat{s}^{2}\left(5-\frac{3}{2} \frac{c^{2}}{\hat{c}^{2}}\right)\right]\right\} .
$$

In Split SUSY the non-SM contributions to $\delta_{V B}$ are suppressed by the large sfermion masses and can be omitted. Once $\hat{\alpha}$ and $\hat{c}^{2} \hat{s}^{2}$ have been computed by means of eqs. (A2) and (A3), it 
is trivial to extract the low-energy boundary conditions on the running electroweak coupling constants $\hat{g}\left(M_{Z}\right)$ and $\hat{g}^{\prime}\left(M_{Z}\right)$.

The top Yukawa coupling is extracted from the physical top mass $M_{t}$ and the running electroweak parameter $\hat{v}$ according to:

$$
\hat{h}_{t}\left(M_{Z}\right) \hat{v}\left(M_{Z}\right)=M_{t}+\Sigma_{t}\left(M_{t}^{2}\right),
$$

where $\Sigma_{t}\left(M_{t}^{2}\right)$ is real part of the top quark self-energy (explicitly given below) computed with external momentum $p^{2}=M_{t}^{2}$, and $\hat{v}$ is defined as:

$$
\hat{v}^{2}(Q)=2 \frac{M_{Z}^{2}+\Pi_{Z Z}\left(M_{Z}^{2}\right)}{\hat{g}^{\prime 2}(Q)+g^{2}(Q)} .
$$

In the low-energy effective theory of Split SUSY the couplings of the Higgs boson to the down-type fermions are SM-like, thus they do not have a large impact on the phenomenology. However, we include all the third-family Yukawa couplings in our analysis for completeness. The Yukawa couplings of the $b$ quark and of the $\tau$ lepton can be extracted from the running masses evaluated at $Q=M_{Z}$ according to:

$$
\hat{h}_{b, \tau}\left(M_{Z}\right) \hat{v}\left(M_{Z}\right)=\bar{m}_{b, \tau}\left(M_{Z}\right)+\Sigma_{b, \tau}^{\mathrm{th}}\left(m_{b, \tau}^{2}\right),
$$

where $\Sigma_{b, \tau}^{\text {th }}\left(m_{b, \tau}^{2}\right)$ contains the weak-scale threshold contributions to the $b$ and $\tau$ self-energies. In order to resum to all orders the potentially large logarithms of the ratio $m_{b, \tau} / M_{Z}$, the running masses $\bar{m}_{b, \tau}$ are evolved up to $Q=M_{Z}$ by means of the RGE of the model with five quarks and $S U(3) \times U(1)_{\mathrm{em}}$ gauge symmetry:

$$
\bar{m}_{b, \tau}\left(M_{Z}\right)=m_{b, \tau}\left(m_{b, \tau}\right)\left(1-\frac{g_{3}^{2}}{8 \pi^{2}} \beta_{s} \log \frac{M_{Z}}{m_{b, \tau}}\right)^{\frac{\gamma_{s}^{b}, \tau}{2 \beta_{s}}}\left(1-\frac{e^{2}}{8 \pi^{2}} \beta_{e} \log \frac{M_{Z}}{m_{b, \tau}}\right)^{\frac{\gamma_{e}, \tau}{2 \beta_{e}}},
$$

where $\left(\beta_{s}, \beta_{e}\right)=(23 / 3,-80 / 9),\left(\gamma_{s}^{b}, \gamma_{e}^{b}\right)=(8,2 / 3)$, and $\left(\gamma_{s}^{\tau}, \gamma_{e}^{\tau}\right)=(0,6)$. The running bottom mass $m_{b}\left(m_{b}\right)$ is taken as input, while the running tau mass $m_{\tau}\left(m_{\tau}\right)$ is extracted from the physical mass $M_{\tau}$ according to:

$$
m_{\tau}\left(m_{\tau}\right)=M_{\tau}\left(1-\frac{e^{2}}{16 \pi^{2}} c_{R}\right)
$$

where $c_{R}=4$ in $\overline{\mathrm{MS}}$ and $c_{R}=5$ in $\overline{\mathrm{DR}}$.

\section{One-loop self-energies}

We now provide explicit formulae for the one-loop self-energies appearing in eqs. (A3), (A4) and (A6)-(A8). The transverse parts of the gauge boson self-energies $\Pi_{V V}$ (with $V=Z, W$ ) can be decomposed in a SM contribution $\Pi_{V V}^{\mathrm{SM}}$ and a chargino/neutralino contribution $\Pi_{V V}^{\chi}$. In the Feynman gauge, that we adopt throughout this appendix, the SM contributions read: 


$$
\begin{aligned}
\frac{16 \pi^{2} \hat{c}^{2}}{\hat{g}^{2}} \Pi_{Z Z}^{\mathrm{SM}}\left(p^{2}\right)= & {\left[m_{Z}^{2} B_{0}\left(m_{Z}, m_{H}\right)-\widetilde{B}_{22}\left(m_{Z}, m_{H}\right)\right]-\left[8 \hat{c}^{4}+\left(\hat{c}^{2}-\hat{s}^{2}\right)^{2}\right] \widetilde{B}_{22}\left(m_{W}, m_{W}\right) } \\
& -2 \hat{c}^{4}\left(2 p^{2}+m_{W}^{2}-m_{Z}^{2} \frac{\hat{s}^{4}}{\hat{c}^{2}}\right) B_{0}\left(m_{W}, m_{W}\right)+\Delta_{Z} \\
& +\sum_{f} N_{c}^{f}\left[\left(g_{f_{L}}^{2}+g_{f_{R}}^{2}\right) H\left(m_{f}, m_{f}\right)-4 g_{f_{L}} g_{f_{R}} m_{f}^{2} B_{0}\left(m_{f}, m_{f}\right)\right],(\mathrm{A} 11) \\
\frac{16 \pi^{2}}{\hat{g}^{2}} \Pi_{W W}^{\mathrm{SM}}\left(p^{2}\right)= & {\left[m_{W}^{2} B_{0}\left(m_{W}, m_{H}\right)-\widetilde{B}_{22}\left(m_{W}, m_{H}\right)\right]-\left(1+8 \hat{c}^{2}\right) \widetilde{B}_{22}\left(m_{Z}, m_{W}\right) } \\
& -\left[\left(4 p^{2}+m_{Z}^{2}+m_{W}^{2}\right) \hat{c}^{2}-m_{Z}^{2} \hat{s}^{4}\right] B_{0}\left(m_{Z}, m_{W}\right)+\Delta_{W} \\
& -\hat{s}^{2}\left[8 \widetilde{B}_{22}\left(m_{W}, 0\right)+4 p^{2} B_{0}\left(m_{W}, 0\right)\right]+\sum_{f} \frac{N_{c}^{f}}{2} H\left(m_{f_{u}}, m_{f_{d}}\right) \cdot(\mathrm{A} 12)
\end{aligned}
$$

The Passarino-Veltman functions $B_{0}, \widetilde{B}_{22}$ and $H$ are defined as in the appendix B of ref. [14]. The Higgs and gauge boson masses appearing in the equations above are interpreted as running masses. The summation in the last line of each equation is over the fermion species, $N_{c}^{f}$ is the color number ( 3 for quarks and 1 for leptons) and $g_{f}=I_{3}^{f}-e_{f} \hat{s}^{2}$ are the weak neutral-current couplings. $\Delta_{Z}$ and $\Delta_{W}$ are $\overline{\mathrm{DR}}-\overline{\mathrm{MS}}$ conversion factors: they are both equal to zero in the $\overline{\mathrm{DR}}$ scheme, while in the $\overline{\mathrm{MS}}$ scheme $\Delta_{Z}=-2 / 3 \hat{c}^{4} p^{2}$ and $\Delta_{W}=-2 / 3 p^{2}$.

The chargino and neutralino contributions to the gauge boson self-energies can be expressed as:

$$
\begin{aligned}
16 \pi^{2} \Pi_{Z Z}^{\chi}\left(p^{2}\right) & =\frac{1}{2} \sum_{i, j}\left[\left(a_{i j Z}^{0}{ }^{2}+b_{i j Z}^{0}{ }^{2}\right) H\left(m_{\chi_{i}^{0}}, m_{\chi_{j}^{0}}\right)+4 a_{i j Z}^{0} b_{i j Z}^{0} m_{\chi_{i}^{0}} m_{\chi_{j}^{0}} B_{0}\left(m_{\chi_{i}^{0}}, m_{\chi_{j}^{0}}\right)\right] \\
& +\sum_{i, j}\left[\left(a_{i j Z}^{+}{ }^{2}+b_{i j Z}^{+}{ }^{2}\right) H\left(m_{\chi_{i}^{+}}, m_{\chi_{j}^{+}}\right)+4 a_{i j Z}^{+} b_{i j Z}^{+} m_{\chi_{i}^{+}} m_{\chi_{j}^{+}} B_{0}\left(m_{\chi_{i}^{+}}, m_{\chi_{j}^{+}}\right)\right], \\
16 \pi^{2} \Pi_{W W}^{\chi}\left(p^{2}\right) & =\sum_{i, j}\left[\left(a_{i j W}{ }^{2}+b_{i j W}{ }^{2}\right) H\left(m_{\chi_{i}^{0}}, m_{\chi_{j}^{+}}\right)+4 a_{i j W} b_{i j W} m_{\chi_{i}^{0}} m_{\chi_{j}^{+}} B_{0}\left(m_{\chi_{i}^{0}}, m_{\chi_{j}^{+}}\right)\right] .
\end{aligned}
$$

In general, we write the Feynman rule for the chargino or neutralino couplings to a gauge boson as $-i \gamma_{\mu}\left(a P_{L}+b P_{R}\right)$, the rule for the couplings to a scalar as $-i\left(a P_{L}+b P_{R}\right)$ and the rule for the coupling to a pseudoscalar as $\left(a P_{L}+b P_{R}\right)$. Under this convention, the chargino and neutralino couplings to the gauge bosons are

$$
\begin{gathered}
a_{i j Z}^{0}=-b_{i j Z}^{0}=\frac{g}{2 \hat{c}}\left(N_{i 3} N_{j 3}-N_{i 4} N_{j 4}\right), \\
a_{i j Z}^{+}=g \hat{c} V_{i 1} V_{j 1}+\frac{g\left(\hat{c}^{2}-\hat{s}^{2}\right)}{2 \hat{c}} V_{i 2} V_{j 2}, \quad b_{i j Z}^{+}=g \hat{c} U_{i 1} U_{j 1}+\frac{g\left(\hat{c}^{2}-\hat{s}^{2}\right)}{2 \hat{c}} U_{i 2} U_{j 2},
\end{gathered}
$$




$$
a_{i j W}=-g \hat{c} N_{i 2} V_{j 1}+\frac{g}{\sqrt{2}} N_{i 4} V_{j 2}, \quad b_{i j W}=-g \hat{c} N_{i 2} U_{j 1}-\frac{g}{\sqrt{2}} N_{i 3} U_{j 2} .
$$

The matrices $N$ and $U, V$ rotate the neutralino and chargino states, respectively, so that the mass matrices $N \mathcal{M}_{N} N^{T}$ and $U \mathcal{M}_{C} V^{T}$ are diagonal. The matrices $\mathcal{M}_{N}$ and $\mathcal{M}_{C}$ are given in eq. (9). We assume that there are no CP-violating phases in the higgsino and gaugino mass parameters, and we choose $N, U$ and $V$ to be real, allowing for negative signs in the chargino and neutralino masses.

The top quark self-energy in eq. (A6) can be expressed as

$$
\begin{aligned}
\frac{16 \pi^{2}}{m_{t}} \Sigma_{t}\left(m_{t}^{2}\right)= & \frac{4 g_{3}^{2}}{3}\left(3 \log \frac{m_{t}^{2}}{Q^{2}}-c_{R}\right)+\frac{4 e^{2}}{9}\left(3 \log \frac{m_{t}^{2}}{Q^{2}}-c_{R}\right) \\
& +\frac{h_{t}^{2}}{2}\left[B_{1}\left(m_{t}, m_{H}\right)+B_{0}\left(m_{t}, m_{H}\right)+B_{1}\left(m_{t}, m_{Z}\right)-B_{0}\left(m_{t}, m_{Z}\right)\right] \\
& +\frac{h_{t}^{2}+h_{b}^{2}}{2} B_{1}\left(m_{b}, m_{W}\right)-\frac{h_{b}^{2}}{2} B_{0}\left(m_{b}, m_{W}\right)+\frac{g^{2}}{2}\left[B_{1}\left(m_{b}, m_{W}\right)+\delta_{R}\right] \\
& +\frac{g^{2}}{\hat{c}^{2}}\left\{\left(g_{t_{L}}^{2}+g_{t_{R}}^{2}\right)\left[B_{1}\left(m_{t}, m_{Z}\right)+\delta_{R}\right]+4 g_{t_{L}} g_{t_{R}}\left[B_{0}\left(m_{t}, m_{Z}\right)+\delta_{R}\right]\right\},
\end{aligned}
$$

where $\left(c_{R}, \delta_{R}\right)$ are equal to $(4,-1 / 2)$ in $\overline{\mathrm{MS}}$ and to $(5,0)$ in $\overline{\mathrm{DR}}$. The Passarino-Veltman functions $B_{0}$ and $B_{1}$ are defined as in the appendix B of ref. [14]. The weak-scale contribution to the bottom quark self-energy $\Sigma_{b}^{\text {th }}\left(m_{b}\right)$, appearing in eq. (A8), can be extracted from eq. (A18) by omitting the first line and replacing everywhere $t \leftrightarrow b$. The analogous quantity for the tau lepton, $\Sigma_{\tau}^{\text {th }}\left(m_{\tau}\right)$, can also be extracted from eq. (A18): one has to omit the first line and the terms proportional to $h_{b}^{2}$ in the third line, then replace everywhere $t \rightarrow \tau$ and $m_{b} \rightarrow 0$.

\section{Corrections to the Higgs mass}

We provide here the formulae for the Higgs mass corrections $\delta^{\mathrm{SM}}(Q)$ and $\delta^{\chi}(Q)$ appearing in eq. (77). The SM contribution reads [24]

$$
\delta^{\mathrm{SM}}(Q)=-\frac{G_{F}}{\sqrt{2}} \frac{M_{Z}^{2}}{16 \pi^{2}}\left[\xi f_{1}(\xi, Q)+f_{0}(\xi, Q)+\xi^{-1} f_{-1}(\xi, Q)\right],
$$

where $\xi=m_{H}^{2} / M_{Z}^{2}$ and the functions $f_{k}(\xi, Q)$ are defined as:

$$
\begin{aligned}
f_{1}(\xi, Q)= & 6 \log \frac{Q^{2}}{m_{H}^{2}}+\frac{3}{2} \log \xi-\frac{1}{2} Z\left(\xi^{-1}\right)-Z\left(c^{2} \xi^{-1}\right)-\log c^{2}+\frac{9}{2}\left(\frac{25}{9}-\frac{\pi}{\sqrt{3}}\right), \quad \text { (A20) } \\
f_{0}(\xi, Q)= & -6 \log \frac{Q^{2}}{M_{Z}^{2}}\left[1+2 c^{2}-2 \frac{m_{t}^{2}}{M_{Z}^{2}}\right]+\frac{3 c^{2} \xi}{\xi-c^{2}} \log \frac{\xi}{c^{2}}+2 Z\left(\xi^{-1}\right)+4 c^{2} Z\left(c^{2} \xi^{-1}\right) \\
& +\left(\frac{3}{s^{2}}+12\right) c^{2} \log c^{2}-\frac{15}{2}\left(1+2 c^{2}\right)-3 \frac{m_{t}^{2}}{M_{Z}^{2}}\left[2 Z\left(\frac{m_{t}^{2}}{m_{H}^{2}}\right)+4 \log \frac{m_{t}^{2}}{M_{Z}^{2}}-5\right],
\end{aligned}
$$




$$
\begin{aligned}
f_{-1}(\xi, Q)= & 6 \log \frac{Q^{2}}{M_{Z}^{2}}\left[1+2 c^{4}-4 \frac{m_{t}^{4}}{M_{Z}^{4}}\right]-6 Z\left(\xi^{-1}\right)-12 c^{4} Z\left(c^{2} \xi^{-1}\right)-12 c^{4} \log c^{2} \\
& +8\left(1+2 c^{4}\right)+24 \frac{m_{t}^{4}}{M_{Z}^{4}}\left[\log \frac{m_{t}^{2}}{M_{Z}^{2}}-2+Z\left(\frac{m_{t}^{2}}{m_{H}^{2}}\right)\right] .
\end{aligned}
$$

In the equations above we define $c^{2}=M_{W}^{2} / M_{Z}^{2}$ in terms of physical masses. On the other hand, as described in section 2.2, we explore the consequences of choosing $m_{t}$ as either the physical or the running top mass. The auxiliary function $Z$ appearing in the equations above is defined as:

$$
Z(x)= \begin{cases}2 A \tan ^{-1}\left(A^{-1}\right) & \left(x>\frac{1}{4}\right) \\ A \log [(1+A) /(1-A)] & \left(x<\frac{1}{4}\right),\end{cases}
$$

where $A=|1-4 x|^{1 / 2}$.

The results of ref. [24] are derived under the assumption that the Higgs quartic coupling $\lambda$ appearing in eq. (7) is expressed in the $\overline{\mathrm{MS}}$ renormalization scheme. If $\lambda$ is expressed in the $\overline{\mathrm{DR}}$ scheme the Higgs mass correction in eq. (A19) is modified as

$$
\delta^{\mathrm{SM}}(Q) \rightarrow \delta^{\mathrm{SM}}(Q)-\frac{g^{2}}{16 \pi^{2}} \frac{M_{W}^{2}}{m_{H}^{2}}\left(1+\frac{1}{2 c^{4}}\right) .
$$

The chargino and neutralino contributions to the Higgs boson self-energy and tadpole, appearing in the correction term $\delta^{\chi}(Q)$ defined in eq. (8), read

$$
\begin{aligned}
16 \pi^{2} \Pi_{H H}^{\chi}\left(p^{2}\right) & =\frac{1}{2} \sum_{i j}\left[\left(a_{i j H}^{0}{ }^{2}+b_{i j H}^{0}{ }^{2}\right) G\left(m_{\chi_{i}^{0}}, m_{\chi_{j}^{0}}\right)-4 a_{i j H}^{0} b_{i j H}^{0} m_{\chi_{i}^{0}} m_{\chi_{j}^{0}} B_{0}\left(m_{\chi_{i}^{0}}, m_{\chi_{j}^{0}}\right)\right] \\
& +\sum_{i j}\left[\left(a_{i j H}^{+}{ }^{2}+b_{i j H}^{+}{ }^{2}\right) G\left(m_{\chi_{i}^{+}}, m_{\chi_{j}^{+}}\right)-4 a_{i j H}^{+} b_{i j H}^{+} m_{\chi_{i}^{+}} m_{\chi_{j}^{+}} B_{0}\left(m_{\chi_{i}^{+}}, m_{\chi_{j}^{+}}\right)\right], \\
16 \pi^{2} T_{H}^{\chi} & =-\sum_{i}\left(a_{i i H}^{0}+b_{i i H}^{0}\right) m_{\chi_{i}^{0}} A_{0}\left(m_{\chi_{i}^{0}}\right)-2 \sum_{i}\left(a_{i i H}^{+}+b_{i i H}^{+}\right) m_{\chi_{i}^{+}} A_{0}\left(m_{\chi_{i}^{+}}\right) .
\end{aligned}
$$

The Passarino-Veltman functions $G, B_{0}$ and $A_{0}$ in the equations above are defined as in the appendix $\mathrm{B}$ of ref. [14]. The chargino and neutralino couplings to the Higgs boson are

$$
\begin{gathered}
a_{i j H}^{0}=b_{i j H}^{0}=\frac{1}{2} N_{i k} N_{j \ell}\left[-\tilde{g}_{d}^{\prime} \delta_{\{k 1} \delta_{\ell 3\}}+\tilde{g}_{u}^{\prime} \delta_{\{k 1} \delta_{\ell \ell\}}+\tilde{g}_{d} \delta_{\{k 2} \delta_{\ell 3\}}-\tilde{g}_{u} \delta_{\{k 2} \delta_{\ell 4\}}\right], \\
a_{i j H}^{+}=b_{j i H}^{+}=\frac{\tilde{g}_{d}}{\sqrt{2}} V_{i 1} U_{j 2}+\frac{\tilde{g}_{u}}{\sqrt{2}} V_{i 2} U_{j 1},
\end{gathered}
$$

where $\tilde{g}_{u, d}$ and $\tilde{g}_{u, d}^{\prime}$ are the effective Higgs-higgsino-gaugino couplings defined in eq. (11). We define $\delta_{\{k i} \delta_{\ell j\}}=\delta_{k i} \delta_{\ell j}+\delta_{k j} \delta_{\ell i}$, where $\delta_{i j}$ is the Kronecker delta and summation over repeated indices is understood. 


\section{Corrections to the chargino and neutralino masses}

We provide here the formulae for the radiative corrections to the chargino and neutralino mass matrices, once again adapting to the Split-SUSY case the results of ref. [14]. The one-loop neutralino mass matrix reads

$$
\mathcal{M}_{N}\left(p^{2}\right)=\mathcal{M}_{N}^{0}+\frac{1}{2}\left(\delta \mathcal{M}_{N}\left(p^{2}\right)+\delta \mathcal{M}_{N}^{T}\left(p^{2}\right)\right)
$$

where

$$
\delta \mathcal{M}_{N}\left(p^{2}\right)=-\Sigma_{R}^{0}\left(p^{2}\right) \mathcal{M}_{N}-\mathcal{M}_{N} \Sigma_{L}^{0}\left(p^{2}\right)-\Sigma_{S}^{0}\left(p^{2}\right) .
$$

The one-loop chargino mass matrix is instead

$$
\mathcal{M}_{C}\left(p^{2}\right)=\mathcal{M}_{C}^{0}-\Sigma_{R}^{+}\left(p^{2}\right) \mathcal{M}_{C}-\mathcal{M}_{C} \Sigma_{L}^{+}\left(p^{2}\right)-\Sigma_{S}^{+}\left(p^{2}\right) .
$$

The tree-level mass matrices $\mathcal{M}_{N}^{0}$ and $\mathcal{M}_{C}^{0}$ are given in eq. (91) and are expressed in terms of minimally renormalized parameters. The neutralino and chargino self-energies $\Sigma_{L, S}^{0}\left(p^{2}\right)$ and $\Sigma_{L, S}^{+}\left(p^{2}\right)$ are $4 \times 4$ and $2 \times 2$ matrices, respectively, and they read

$$
\begin{aligned}
& 16 \pi^{2} \Sigma_{L i j}^{0}=\sum_{k=1}^{2}\left\{\tilde{a}_{i k G^{+}}^{0} \tilde{a}_{j k G^{+}}^{0} B_{1}\left(m_{\chi_{k}^{+}}, m_{W}\right)+2 \tilde{a}_{i k W}^{0} \tilde{a}_{j k W}^{0}\left[B_{1}\left(m_{\chi_{k}^{+}}, m_{W}\right)+\delta_{R}\right]\right\} \\
& +\frac{1}{2} \sum_{k=1}^{4}\left\{\tilde{a}_{i k G^{0}}^{0} \tilde{a}_{j k G^{0}}^{0} B_{1}\left(m_{\chi_{k}^{0}}, m_{Z}\right)+\tilde{a}_{i k H}^{0} \tilde{a}_{j k H}^{0} B_{1}\left(m_{\chi_{k}^{0}}, m_{H}\right)\right. \\
& \left.+2 \tilde{a}_{i k Z}^{0} \tilde{a}_{j k Z}^{0}\left[B_{1}\left(m_{\chi_{k}^{0}}, m_{Z}\right)+\delta_{R}\right]\right\}, \\
& 16 \pi^{2} \Sigma_{S i j}^{0}=2 \sum_{k=1}^{2} m_{\chi_{k}^{+}}\left\{\tilde{b}_{i k G^{+}}^{0} \tilde{a}_{j k G^{+}}^{0} B_{0}\left(m_{\chi_{k}^{+}}, m_{W}\right)-4 \tilde{b}_{i k W}^{0} \tilde{a}_{j k W}^{0}\left[B_{0}\left(m_{\chi_{k}^{+}}, m_{W}\right)+\delta_{R}\right]\right\} \\
& +\quad \sum_{k=1}^{4} m_{\chi_{k}^{0}}\left\{\tilde{b}_{i k G^{0}}^{0} \tilde{a}_{j k G^{0}}^{0} B_{0}\left(m_{\chi_{k}^{0}}, m_{Z}\right)+\tilde{b}_{i k H}^{0} \tilde{a}_{j k H}^{0} B_{0}\left(m_{\chi_{k}^{0}}, m_{H}\right)\right. \\
& \left.-4 \tilde{b}_{i k Z}^{0} \tilde{a}_{j k Z}^{0}\left[B_{0}\left(m_{\chi_{k}^{0}}, m_{Z}\right)+\delta_{R}\right]\right\} \\
& 16 \pi^{2} \Sigma_{L i j}^{+}=\frac{1}{2} \sum_{k=1}^{4}\left\{\tilde{a}_{k i G^{+}}^{+} \tilde{a}_{k j G^{+}}^{+} B_{1}\left(m_{\chi_{k}^{0}}, m_{W}\right)+2 \tilde{a}_{k i W}^{+} \tilde{a}_{k j W}^{+}\left[B_{1}\left(m_{\chi_{k}^{0}}, m_{W}\right)+\delta_{R}\right]\right\} \\
& +\frac{1}{2} \sum_{k=1}^{2}\left\{\tilde{a}_{i k G^{0}}^{+} \tilde{a}_{j k G^{0}}^{+} B_{1}\left(m_{\chi_{k}^{+}}, m_{Z}\right)+\tilde{a}_{i k H}^{+} \tilde{a}_{j k H}^{+} B_{1}\left(m_{\chi_{k}^{+}}, m_{H}\right)\right. \\
& \left.+2 \tilde{a}_{i k Z}^{+} \tilde{a}_{j k Z}^{+}\left[B_{1}\left(m_{\chi_{k}^{+}}, m_{Z}\right)+\delta_{R}\right]+2 \tilde{a}_{i k \gamma}^{+} \tilde{a}_{j k \gamma}^{+}\left[B_{1}\left(m_{\chi_{k}^{+}}, 0\right)+\delta_{R}\right]\right\},
\end{aligned}
$$




$$
\begin{aligned}
16 \pi^{2} \Sigma_{S i j}^{+}= & \sum_{k=1}^{4} m_{\chi_{k}^{0}}\left\{\tilde{b}_{k i G^{+}}^{+} \tilde{a}_{k j G^{+}}^{+} B_{0}\left(m_{\chi_{k}^{0}}, m_{W}\right)-4 \tilde{b}_{k i W}^{+} \tilde{a}_{k j W}^{+}\left[B_{0}\left(m_{\chi_{k}^{0}}, m_{W}\right)+\delta_{R}\right]\right\} \\
+ & \sum_{k=1}^{2} m_{\chi_{k}^{+}}\left\{\tilde{b}_{i k G^{0}}^{+} \tilde{a}_{j k G^{0}}^{+} B_{0}\left(m_{\chi_{k}^{+}}, m_{Z}\right)+\tilde{b}_{i k H}^{+} \tilde{a}_{j k H}^{+} B_{0}\left(m_{\chi_{k}^{+}}, m_{H}\right)\right. \\
& \left.\quad-4 \tilde{b}_{i k Z}^{+} \tilde{a}_{j k Z}^{+}\left[B_{0}\left(m_{\chi_{k}^{+}}, m_{Z}\right)+\delta_{R}\right]-4 \tilde{b}_{i k \gamma}^{+} \tilde{a}_{j k \gamma}^{+}\left[B_{0}\left(m_{\chi_{k}^{+}}, 0\right)+\delta_{R}\right]\right\},
\end{aligned}
$$

where $\delta_{R}=0$ in $\overline{\mathrm{DR}}$ and $\delta_{R}=-1 / 2$ in $\overline{\mathrm{MS}}$. The formulae for the self-energies $\Sigma_{R}^{0}$ and $\Sigma_{R}^{+}$ can be obtained from those for $\Sigma_{L}^{0}$ and $\Sigma_{L}^{+}$, respectively, by replacing $\tilde{a} \rightarrow \tilde{b}$ in eqs. (A32) and (A34). In the equations above we denote by $\tilde{a}_{i j \Phi}$ and $\tilde{b}_{i j \Phi}$ the couplings of a bosonic field $\Phi=\left(Z, W, \gamma, H, G^{0}, G^{+}\right)$with one rotated neutralino (or chargino) mass eigenstate and one unrotated neutralino (or chargino) gauge eigenstate.

In particular, the couplings of an unrotated neutralino $\psi_{i}^{0}$, a rotated chargino $\chi_{j}^{+}$and a charged pseudo-Goldstone boson read

$$
\begin{aligned}
& \tilde{a}_{i j G^{+}}^{0}=U_{j k}\left(\frac{\tilde{g}_{d}^{\prime}}{\sqrt{2}} \delta_{i 1} \delta_{k 2}+\frac{\tilde{g}_{d}}{\sqrt{2}} \delta_{i 2} \delta_{k 2}-\tilde{g}_{d} \delta_{i 3} \delta_{k 1}\right) \\
& \tilde{b}_{i j G^{+}}^{0}=V_{j k}\left(\frac{\tilde{g}_{u}^{\prime}}{\sqrt{2}} \delta_{i 1} \delta_{k 2}+\frac{\tilde{g}_{u}}{\sqrt{2}} \delta_{i 2} \delta_{k 2}+\tilde{g}_{u} \delta_{i 4} \delta_{k 1}\right) .
\end{aligned}
$$

The couplings of an unrotated neutralino $\psi_{i}^{0}$, a rotated chargino $\chi_{j}^{+}$and a $\mathrm{W}$ boson read

$$
\begin{aligned}
& \tilde{a}_{i j W}^{0}=g V_{j k}\left(-\delta_{i 2} \delta_{k 1}+\frac{1}{\sqrt{2}} \delta_{i 4} \delta_{k 2}\right), \\
& \tilde{b}_{i j W}^{0}=g U_{j k}\left(-\delta_{i 2} \delta_{k 1}-\frac{1}{\sqrt{2}} \delta_{i 3} \delta_{k 2}\right) .
\end{aligned}
$$

The couplings of an unrotated neutralino $\psi_{i}^{0}$, a rotated neutralino $\chi_{j}^{0}$ and a neutral pseudoGoldstone boson read

$$
\tilde{a}_{i j G^{0}}^{0}=-\tilde{b}_{i j G^{0}}^{0}=\frac{1}{2} N_{j k}\left(-\tilde{g}_{d}^{\prime} \delta_{\{i 1} \delta_{k 3\}}-\tilde{g}_{u}^{\prime} \delta_{\{i 1} \delta_{k 4\}}+\tilde{g}_{d} \delta_{\{i 2} \delta_{k 3\}}+\tilde{g}_{u} \delta_{\{i 2} \delta_{k 4\}}\right) .
$$

The couplings of an unrotated neutralino $\psi_{i}^{0}$, a rotated neutralino $\chi_{j}^{0}$ and a Higgs boson read

$$
\tilde{a}_{i j H}^{0}=\quad \tilde{b}_{i j H}^{0}=\frac{1}{2} N_{j k}\left(-\tilde{g}_{d}^{\prime} \delta_{\{i 1} \delta_{k 3\}}+\tilde{g}_{u}^{\prime} \delta_{\{i 1} \delta_{k 4\}}+\tilde{g}_{d} \delta_{\{i 2} \delta_{k 3\}}-\tilde{g}_{u} \delta_{\{i 2} \delta_{k 4\}}\right) .
$$

The couplings of an unrotated neutralino $\psi_{i}^{0}$, a rotated neutralino $\chi_{j}^{0}$ and a $Z$ boson read

$$
\tilde{a}_{i j Z}^{0}=-\tilde{b}_{i j Z}^{0}=\frac{g}{2 \hat{c}} N_{j k}\left(\delta_{i 3} \delta_{k 3}-\delta_{i 4} \delta_{k 4}\right) .
$$


The couplings of a rotated neutralino $\chi_{i}^{0}$, an unrotated chargino $\psi_{j}^{+}$and a charged pseudoGoldstone boson read

$$
\begin{aligned}
& \tilde{a}_{i j G^{+}}^{+}=N_{i k}\left(\frac{\tilde{g}_{u}^{\prime}}{\sqrt{2}} \delta_{k 1} \delta_{j 2}+\frac{\tilde{g}_{u}}{\sqrt{2}} \delta_{k 2} \delta_{j 2}+\tilde{g}_{u} \delta_{k 4} \delta_{j 1}\right) \\
& \tilde{b}_{i j G^{+}}^{+}=N_{i k}\left(\frac{\tilde{g}_{d}^{\prime}}{\sqrt{2}} \delta_{k 1} \delta_{j 2}+\frac{\tilde{g}_{d}}{\sqrt{2}} \delta_{k 2} \delta_{j 2}-\tilde{g}_{d} \delta_{k 3} \delta_{j 1}\right) .
\end{aligned}
$$

The couplings of a rotated neutralino $\chi_{i}^{0}$, an unrotated chargino $\psi_{j}^{+}$and a $\mathrm{W}$ boson read

$$
\begin{aligned}
& \tilde{a}_{i j W}^{+}=g N_{i k}\left(-\delta_{k 2} \delta_{j 1}+\frac{1}{\sqrt{2}} \delta_{k 4} \delta_{j 2}\right), \\
& \tilde{b}_{i j W}^{+}=g N_{i k}\left(-\delta_{k 2} \delta_{j 1}-\frac{1}{\sqrt{2}} \delta_{k 3} \delta_{j 2}\right) .
\end{aligned}
$$

The couplings of an unrotated chargino $\psi_{i}^{+}$, a rotated chargino $\chi_{j}^{+}$and a neutral pseudoGoldstone boson read

$$
\begin{aligned}
& \tilde{a}_{i j G^{0}}^{+}=\frac{1}{\sqrt{2}} U_{j k}\left(\tilde{g}_{d} \delta_{i 1} \delta_{k 2}-\tilde{g}_{u} \delta_{i 2} \delta_{k 1}\right) \\
& \tilde{b}_{i j G^{0}}^{+}=\frac{1}{\sqrt{2}} V_{j k}\left(\tilde{g}_{u} \delta_{i 1} \delta_{k 2}-\tilde{g}_{d} \delta_{i 2} \delta_{k 1}\right) .
\end{aligned}
$$

The couplings of an unrotated chargino $\psi_{i}^{+}$, a rotated chargino $\chi_{j}^{+}$and a Higgs boson read

$$
\begin{aligned}
& \tilde{a}_{i j H}^{+}=\frac{1}{\sqrt{2}} U_{j k}\left(\tilde{g}_{d} \delta_{i 1} \delta_{k 2}+\tilde{g}_{u} \delta_{i 2} \delta_{k 1}\right), \\
& \tilde{b}_{i j H}^{+}=\frac{1}{\sqrt{2}} V_{j k}\left(\tilde{g}_{u} \delta_{i 1} \delta_{k 2}+\tilde{g}_{d} \delta_{i 2} \delta_{k 1}\right) .
\end{aligned}
$$

The couplings of an unrotated chargino $\psi_{i}^{+}$, a rotated chargino $\chi_{j}^{+}$and a $Z$ boson read

$$
\begin{aligned}
& \tilde{a}_{i j Z}^{+}=g \hat{c} V_{j k}\left(\delta_{i 1} \delta_{k 1}+\frac{\hat{c}^{2}-\hat{s}^{2}}{2 \hat{c}^{2}} \delta_{i 2} \delta_{k 2}\right), \\
& \tilde{b}_{i j Z}^{+}=g \hat{c} U_{j k}\left(\delta_{i 1} \delta_{k 1}+\frac{\hat{c}^{2}-\hat{s}^{2}}{2 \hat{c}^{2}} \delta_{i 2} \delta_{k 2}\right) .
\end{aligned}
$$

Finally, the couplings of an unrotated chargino $\psi_{i}^{+}$, a rotated chargino $\chi_{j}^{+}$and a photon read

$$
\begin{aligned}
& \tilde{a}_{i j \gamma}^{+}=e V_{j k}\left(\delta_{i 1} \delta_{k 1}+\delta_{i 2} \delta_{k 2}\right), \\
& \tilde{b}_{i j \gamma}^{+}=e U_{j k}\left(\delta_{i 1} \delta_{k 1}+\delta_{i 2} \delta_{k 2}\right) .
\end{aligned}
$$

The self-energies in eqs. (A29)-(A31) induce an external momentum dependence in the neutralino and chargino mass matrices. We compute each of the physical neutralino and chargino masses by diagonalizing the one-loop mass matrix with the external momenta in the Passarino-Veltman functions set equal to the tree-level mass of the corresponding particle. 


\section{More one-loop translations from $\overline{\mathrm{DR}}$ to $\overline{\mathrm{MS}}$}

Beyond tree level the boundary conditions on the quartic Higgs coupling and on the Higgshiggsino-gaugino couplings given in eqs. (3)-(5) are valid only in the $\overline{\mathrm{DR}}$ renormalization scheme. The $\overline{\mathrm{MS}}$ scheme breaks supersymmetry, therefore in that scheme the SUSY relations between the gauge couplings and the gaugino couplings and those between the gauge couplings and the quartic scalar couplings are not preserved [50]. In the $\overline{\mathrm{MS}}$ scheme the boundary conditions in eqs. (3)-(15) are modified as 2

$$
\begin{aligned}
\lambda\left(M_{S}\right) & =\frac{1}{4}\left[g^{2}\left(M_{S}\right)+g^{\prime 2}\left(M_{S}\right)\right] \cos ^{2} 2 \beta-\frac{g^{4}}{32 \pi^{2}}\left(1+\frac{1}{2 c^{4}}\right)+\Delta_{\mathrm{th}} \lambda, \\
\tilde{g}_{u}\left(M_{S}\right) & =g\left(M_{S}\right) \sin \beta\left[1+\frac{g^{2}}{16 \pi^{2}}\left(\frac{13}{12}-\frac{1}{8 c^{2}}\right)\right], \\
\tilde{g}_{d}\left(M_{S}\right) & =g\left(M_{S}\right) \cos \beta\left[1+\frac{g^{2}}{16 \pi^{2}}\left(\frac{13}{12}-\frac{1}{8 c^{2}}\right)\right], \\
\tilde{g}_{u}^{\prime}\left(M_{S}\right) & =g^{\prime}\left(M_{S}\right) \sin \beta\left[1-\frac{g^{2}}{16 \pi^{2}}\left(\frac{1}{4}+\frac{1}{8 c^{2}}\right)\right], \\
\tilde{g}_{d}^{\prime}\left(M_{S}\right) & =g^{\prime}\left(M_{S}\right) \cos \beta\left[1-\frac{g^{2}}{16 \pi^{2}}\left(\frac{1}{4}+\frac{1}{8 c^{2}}\right)\right],
\end{aligned}
$$

where $\Delta_{\text {th }} \lambda$ is the $\mathcal{O}\left(h_{t}^{4}\right)$ threshold correction defined in eq. (6).

For completeness we conclude this section by providing the relations between the $\overline{\mathrm{DR}}$ and $\overline{\mathrm{MS}}$ definitions of the gaugino and higgsino mass terms:

$$
\begin{aligned}
M_{1}^{\overline{\mathrm{MS}}} & =M_{1}^{\overline{\mathrm{DR}}} \\
M_{2}^{\overline{\mathrm{MS}}} & =M_{2}^{\overline{\mathrm{DR}}}\left[1+\frac{g^{2}}{8 \pi^{2}}\right], \\
M_{3}^{\overline{\mathrm{MS}}} & =M_{3}^{\overline{\mathrm{DR}}}\left[1+\frac{3 g_{s}^{2}}{16 \pi^{2}}\right], \\
\mu^{\overline{\mathrm{MS}}} & =\mu^{\overline{\mathrm{DR}}}\left[1+\frac{g^{2}}{16 \pi^{2}}\left(\frac{1}{2}+\frac{1}{4 c^{2}}\right)\right] .
\end{aligned}
$$

\footnotetext{
${ }^{2}$ Note Added: in eq. (A55) the SU(2) gauge coupling $g\left(M_{S}\right)$ entering the tree-level part of the boundary condition on $\lambda$ has to be interpreted as the $\overline{\mathrm{DR}}$ coupling. By contrast, in eqs. (A56) and (A57) $g\left(M_{S}\right)$ has to be interpreted as the $\overline{\mathrm{MS}}$ coupling. If the coupling $g\left(M_{S}\right)$ in the tree-level boundary condition on $\lambda$ is expressed in the $\overline{\mathrm{MS}}$ scheme, there is an additional shift in eq. (A55) amounting to $+g^{4} \cos ^{2} 2 \beta /\left(96 \pi^{2}\right)$.
} 


\section{References}

[1] J. Wess and B. Zumino, Nucl. Phys. B 70 (1974) 39; J. Wess and J. Bagger, Supersymmetry and Supergravity, Princeton Series in Physics, New Jersey, 1992.

[2] E. Witten, Nucl. Phys. B 188 (1981) 513, Nucl. Phys. B 202 (1982) 253; N. Sakai, Z. Phys. C 11 (1981) 153; S. Dimopoulos and H. Georgi, Nucl. Phys. B 193 (1981) 150; R. K. Kaul and P. Majumdar, Nucl. Phys. B 199 (1982) 36.

[3] See e.g., R. Barbieri and G. F. Giudice, Nucl. Phys. B 306 (1988) 63.

[4] J. R. Ellis, S. Kelley and D. V. Nanopoulos, Phys. Lett. B 260 (1991) 131; U. Amaldi, W. de Boer and H. Furstenau, Phys. Lett. B 260 (1991) 447; P. Langacker and M. x. Luo, Phys. Rev. D 44 (1991) 817; C. Giunti, C. W. Kim and U. W. Lee, Mod. Phys. Lett. A 6 (1991) 1745.

[5] For detailed reviews on SUSY Dark Matter, see: G. Jungman, M. Kamionkowski and K. Griest, Phys. Rept. 267 (1996) 195 [arXiv:hep-ph/9506380]; G. Bertone, D. Hooper and J. Silk, Phys. Rept. 405 (2005) 279 [arXiv:hep-ph/0404175].

[6] N. Arkani-Hamed and S. Dimopoulos, JHEP 0506 (2005) 073 [arXiv:hep-th/0405159].

[7] G. F. Giudice and A. Romanino, Nucl. Phys. B 699 (2004) 65 [Erratum-ibid. B 706 (2005) 65] [arXiv:hep-ph/0406088].

[8] J. D. Wells, Phys. Rev. D 71 (2005) 015013 [arXiv:hep-ph/0411041].

[9] H. E. Haber and G. L. Kane, Phys. Rept. 117 (1985) 75; H. P. Nilles, Phys. Rept. 110 (1984) 1; S. P. Martin, arXiv:hep-ph/9709356; M. Drees, R. M. Godbole and P. Roy, Theory and Phenomenology of Sparticles, World Scientific, Spring 2004.

[10] A. Djouadi and S. Rosiers-Lees (conv.) et al., Summary Report of the MSSM Working Group for the "GDR-Supersymétrie", hep-ph/9901246.

[11] A. Djouadi, J. L. Kneur and G. Moultaka, SuSpect, arXiv:hep-ph/0211331, available from the web site: http://www.lpta.univ-montp2.fr/ ${ }^{\sim k n e u r / S u s p e c t ~ . ~}$

[12] H. Baer, F. E. Paige, S. D. Protopopescu and X. Tata, ISAJET, arXiv:hep-ph/0001086; B. C. Allanach, SoftSusy, Comput. Phys. Commun. 143 (2002) 305 [arXiv:hepph/0104145]; W. Porod, SPheno, Comput. Phys. Commun. 153 (2003) 275 [arXiv:hep$\mathrm{ph} / 0301101]$.

[13] A. Arvanitaki, C. Davis, P. W. Graham and J. G. Wacker, Phys. Rev. D 70 (2004) 117703 [arXiv:hep-ph/0406034]. 
[14] D. M. Pierce, J. A. Bagger, K. T. Matchev and R. j. Zhang, Nucl. Phys. B 491 (1997) 3 [arXiv:hep-ph/9606211].

[15] A. Pierce, Phys. Rev. D 70 (2004) 075006 [arXiv:hep-ph/0406144]; A. Arvanitaki and P. W. Graham, Phys. Rev. D 72 (2005) 055010 [arXiv:hep-ph/0411376].

[16] A. Djouadi, J. Kalinowski and M. Spira, Comput. Phys. Commun. 108 (1998) 56 [arXiv:hep-ph/9704448].

[17] M. Muhlleitner, A. Djouadi and Y. Mambrini, Comput. Phys. Commun. 168 (2005) 46 [arXiv:hep-ph/0311167].

[18] A. H. Chamseddine, R. Arnowitt and P. Nath, Phys. Rev. Lett. 49 (1982) 970; R. Barbieri, S. Ferrara and C. A. Savoy, Phys. Lett. B 119 (1982) 343. L. J. Hall, J. D. Lykken and S. Weinberg, Phys. Rev. D 27 (1983) 2359; N. Ohta, Prog. Theor. Phys. 70 (1983) 542.

[19] J. Amundson et al., In the Proceedings of 1996 DPF / DPB Summer Study on New Directions for High-Energy Physics (Snowmass 96), Snowmass, Colorado, 25 Jun - 12 Jul 1996, pp SUP106 [arXiv:hep-ph/9609374]; G. Anderson, C. H. Chen, J. F. Gunion, J. D. Lykken, T. Moroi and Y. Yamada, ibid. pp SUP107 [arXiv:hep-ph/9609457]; G. Anderson, H. Baer, C.H. Chen and X. Tata, Phys. Rev. D61 (2000) 095005 [arXiv:hep-ph/9903370];

[20] C. T. Hill, Phys. Lett. B 135 (1984) 47; Q. Shafi and C. Wetterich, Phys. Rev. Lett. 52 (1984) 875; J. R. Ellis, K. Enqvist, D. V. Nanopoulos and K. Tamvakis, Phys. Lett. B 155 (1985) 381; M. Drees, Phys. Lett. B 158 (1985) 409. K. Huitu, Y. Kawamura, T. Kobayashi and K. Puolamaki, Phys. Lett. B 468 (1999) 111 [arXiv:hep-ph/9909227].

[21] A. Brignole, L. E. Ibanez and C. Munoz, Nucl. Phys. B 422 (1994) 125 [Erratum-ibid. B 436 (1995) 747] [arXiv:hep-ph/9308271]; C. H. Chen, M. Drees and J. F. Gunion, Phys. Rev. D 55 (1997) 330 [Erratum-ibid. D 60 (1999) 039901] [arXiv:hep-ph/9607421]. P. Binetruy, M. K. Gaillard and B. D. Nelson, Nucl. Phys. B 604 (2001) 32 [arXiv:hep$\mathrm{ph} / 0011081]$.

[22] W. M. Yao et al. [Particle Data Group], J. Phys. G 33 (2006) 1.

[23] [CDF Collaboration], arXiv:hep-ex/0703034.

[24] A. Sirlin and R. Zucchini, Nucl. Phys. B 266 (1986) 389.

[25] M. Binger, Phys. Rev. D 73 (2006) 095001 [arXiv:hep-ph/0408240].

[26] For a recent review on Higgs physics in the SM and in the MSSM see, e.g., A. Djouadi, arXiv:hep-ph/0503172, arXiv:hep-ph/0503173. 
[27] K. Choi and H. P. Nilles, arXiv:hep-ph/0702146.

[28] L. Randall and R. Sundrum, Nucl. Phys. B 557 (1999) 79 [arXiv:hep-th/9810155]; G. F. Giudice, M. A. Luty, H. Murayama and R. Rattazzi, JHEP 9812 (1998) 027 [arXiv:hep-ph/9810442]; J. A. Bagger, T. Moroi and E. Poppitz, JHEP 0004 (2000) 009 [arXiv:hep-th/9911029].

[29] K. Choi, A. Falkowski, H. P. Nilles, M. Olechowski and S. Pokorski, JHEP 0411 (2004) 076 [arXiv:hep-th/0411066]. K. Choi, A. Falkowski, H. P. Nilles and M. Olechowski, Nucl. Phys. B 718 (2005) 113 [arXiv:hep-th/0503216]; M. Endo, M. Yamaguchi and K. Yoshioka, Phys. Rev. D 72 (2005) 015004 [arXiv:hep-ph/0504036].

[30] S. Kachru, R. Kallosh, A. Linde and S. P. Trivedi, Phys. Rev. D 68 (2003) 046005 [arXiv:hep-th/0301240].

[31] D. N. Spergel et al. [WMAP Collaboration], arXiv:astro-ph/0603449.

[32] M. Toharia and J. D. Wells, JHEP 0602 (2006) 015 [arXiv:hep-ph/0503175].

[33] A. Arvanitaki, C. Davis, P. W. Graham, A. Pierce and J. G. Wacker, Phys. Rev. D 72 (2005) 075011 [arXiv:hep-ph/0504210].

[34] P. Gambino, G. F. Giudice and P. Slavich, Nucl. Phys. B 726 (2005) 35 [arXiv:hep$\mathrm{ph} / 0506214]$.

[35] A. Corsetti and P. Nath, Phys. Rev. D 64 (2001) 125010 [arXiv:hep-ph/0003186]; A. Birkedal-Hansen and B. D. Nelson, Phys. Rev. D 64 (2001) 015008 [arXiv:hepph/0102075], Phys. Rev. D 67 (2003) 095006 [arXiv:hep-ph/0211071]; H. Baer, C. Balazs, A. Belyaev, R. Dermisek, A. Mafi and A. Mustafayev, JHEP 0205 (2002) 061 [arXiv:hep-ph/0204108]; V. Bertin, E. Nezri and J. Orloff, JHEP 0302 (2003) 046 [arXiv:hep-ph/0210034]; U. Chattopadhyay and D. P. Roy, Phys. Rev. D 68 (2003) 033010 [arXiv:hep-ph/0304108]; D. G. Cerdeno and C. Munoz, JHEP 0410 (2004) 015 [arXiv:hep-ph/0405057]; A. Bottino, F. Donato, N. Fornengo and S. Scopel, Phys. Rev. D 70 (2004) 015005 [arXiv:hep-ph/0401186]; S. Profumo and C. E. Yaguna, Phys. Rev. D 69 (2004) 115009 [arXiv:hep-ph/0402208], Phys. Rev. D 70 (2004) 095004 [arXiv:hep-ph/0407036]; G. Belanger, F. Boudjema, A. Cottrant, A. Pukhov and A. Semenov, Nucl. Phys. B 706 (2005) 411 [arXiv:hep-ph/0407218]; Y. Mambrini and E. Nezri, arXiv:hep-ph/0507263; U. Chattopadhyay, D. Choudhury, M. Drees, P. Konar and D. P. Roy, Phys. Lett. B 632 (2006) 114 [arXiv:hep-ph/0508098]; S. F. King and J. P. Roberts, JHEP 0609 (2006) 036 [arXiv:hep-ph/0603095], JHEP 0701 (2007) 024 [arXiv:hep-ph/0608135]; H. Baer, A. Mustafayev, E. K. Park, S. Profumo and X. Tata, JHEP 0604 (2006) 041 [arXiv:hep-ph/0603197]; H. Baer, 
A. Mustafayev, S. Profumo and X. Tata, Phys. Rev. D 75 (2007) 035004 [arXiv:hepph/0610154]; H. Baer, E. K. Park, X. Tata and T. T. Wang, arXiv:hep-ph/0703024; K. J. Bae, R. Dermisek, H. D. Kim and I. W. Kim, arXiv:hep-ph/0702041.

[36] A. Masiero, S. Profumo and P. Ullio, Nucl. Phys. B 712 (2005) 86 [arXiv:hepph/0412058]; N. Arkani-Hamed, A. Delgado and G. F. Giudice, Nucl. Phys. B 741 (2006) 108 [arXiv:hep-ph/0601041]; R. Mahbubani and L. Senatore, Phys. Rev. D 73 (2006) 043510 [arXiv:hep-ph/0510064]; M. Masip and I. Mastromatteo, Phys. Rev. D 73 (2006) 015007 [arXiv:hep-ph/0510311]; A. Provenza, M. Quiros and P. Ullio, JCAP 0612 (2006) 007 [arXiv:hep-ph/0609059]. F. Wang, W. Wang and J. M. Yang, Eur. Phys. J. C 46 (2006) 521 [arXiv:hep-ph/0512133].

[37] S. P. Martin, K. Tobe and J. D. Wells, Phys. Rev. D 71 (2005) 073014 [arXiv:hep$\mathrm{ph} / 0412424]$.

[38] For recent analyses, see: H. Baer, A. Belyaev, T. Krupovnickas and X. Tata, JHEP 0402 (2004) 007 [arXiv:hep-ph/0311351]; H. Baer, T. Krupovnickas and X. Tata, JHEP 0406 (2004) 061 [arXiv:hep-ph/0405058]; A. Djouadi, M. Drees and J. L. Kneur, Phys. Lett. B 624 (2005) 60 [arXiv:hep-ph/0504090] and JHEP 0603 (2006) 033 [arXiv:hep-ph/0602001].

[39] G. Pocsik and T. Torma, Z. Phys. C 6 (1980) 1; T. G. Rizzo, Phys. Rev. D 22 (1980) 722; W. Y. Keung and W. J. Marciano, Phys. Rev. D 30 (1984) 248; A. Djouadi, J. Kalinowski and P. M. Zerwas, Z. Phys. C 70 (1996) 435 [arXiv:hep-ph/9511342].

[40] The routine is based on the work done in: M. Drees and M. M. Nojiri, Phys. Rev. D 45 (1992) 2482; Phys. Rev. D 47 (1993) 376 [arXiv:hep-ph/9207234] and Phys. Rev. D 48 (1993) 3483 [arXiv:hep-ph/9307208].

[41] G. Belanger, F. Boudjema, A. Pukhov and A. Semenov, micrOMEGAs, Comput. Phys. Commun. 149 (2002) 103 [arXiv:hep-ph/0112278] and Comput. Phys. Commun. 176 (2007) 367 [arXiv:hep-ph/0607059].

[42] A. Djouadi, M. Spira and P. M. Zerwas, Phys. Lett. B 264 (1991) 440; M. Spira, A. Djouadi, D. Graudenz and P. M. Zerwas, Nucl. Phys. B 453 (1995) 17 [arXiv:hepph/9504378]; A. Djouadi, M. Spira and P. M. Zerwas, Z. Phys. C 70 (1996) 427 [arXiv:hep-ph/9511344].

[43] K. Griest and H. E. Haber, Phys. Rev. D 37 (1988) 719; J. Kalinowski, B. Grzadkowski and S. Pokorski, Phys. Lett. B 241 (1990) 534; A. Djouadi, J. Kalinowski and P. M. Zerwas, Z. Phys. C 57 (1993) 569; A. Djouadi, P. Janot, J. Kalinowski and P. M. Zerwas, Phys. Lett. B 376 (1996) 220 [arXiv:hep-ph/9603368]; A. Djouadi, 
J. Kalinowski, P. Ohmann and P. M. Zerwas, Z. Phys. C 74 (1997) 93 [arXiv:hepph/9605339]; J. F. Gunion and J. Kelly, Phys. Rev. D 56 (1997) 1730 [arXiv:hep$\mathrm{ph} / 9610495]$.

[44] P. Kalyniak, R. Bates and J. N. Ng, Phys. Rev. D 33 (1986) 755 and Phys. Rev. D 34 (1986) 172; J. F. Gunion, G. Gamberini and S. F. Novaes, Phys. Rev. D 38 (1988) 3481; T. M. Aliev and Yu. M. Kasumzade, Sov. J. Nucl. Phys. 47 (1988) 293 [Yad. Fiz. 47 (1988) 464]; A. Djouadi, V. Driesen, W. Hollik and J. I. Illana, Eur. Phys. J. C 1 (1998) 149 [arXiv:hep-ph/9612362]; A. Djouadi, Phys. Lett. B 435 (1998) 101 [arXiv:hep-ph/9806315].

[45] M. A. Diaz and P. F. Perez, J. Phys. G 31 (2005) 563 [arXiv:hep-ph/0412066]. S. K. Gupta, B. Mukhopadhyaya and S. K. Rai, Phys. Rev. D 73 (2006) 075006 [arXiv:hep-ph/0510306].

[46] A. Bartl, W. Majerotto and W. Porod, Z. Phys. C 64 (1994) 499 [Erratum-ibid. C 68 (1995) 518], Phys. Lett. B 465 (1999) 187 [arXiv:hep-ph/9907377]; H. Baer, C. h. Chen, M. Drees, F. Paige and X. Tata, Phys. Rev. Lett. 79 (1997) 986 [arXiv:hep-ph/9704457], Phys. Rev. D 59 (1999) 055014 [arXiv:hep-ph/9809223]; A. Djouadi and Y. Mambrini, Phys. Lett. B 493 (2000) 120 [arXiv:hep-ph/0007174]; A. Djouadi, Y. Mambrini and M. Muhlleitner, Eur. Phys. J. C 20 (2001) 563 [arXiv:hep-ph/0104115].

[47] J. F. Gunion and H. E. Haber, Phys. Rev. D 37 (1988) 2515; J. F. Gunion, H. E. Haber, R. M. Barnett, M. Drees, D. Karatas, X. Tata and H. Baer, Int. J. Mod. Phys. A 2 (1987) 1145; H. Baer, M. Bisset, X. Tata and J. Woodside, Phys. Rev. D 46 (1992) 303; A. Datta, A. Djouadi, M. Guchait and Y. Mambrini, Phys. Rev. D 65 (2002) 015007 [arXiv:hep-ph/0107271]; A. Datta, A. Djouadi, M. Guchait and F. Moortgat, Nucl. Phys. B 681 (2004) 31 [arXiv:hep-ph/0303095].

[48] H. E. Haber and D. Wyler, Nucl. Phys. B 323 (1989) 267; S. Ambrosanio and B. Mele, Phys. Rev. D 55 (1997) 1399 [Erratum-ibid. D 56 (1997) 3157] [arXiv:hep-ph/9609212]; H. Baer and T. Krupovnickas, JHEP 0209 (2002) 038 [arXiv:hep-ph/0208277]; K. Cheung and J. Song, Phys. Rev. D 72 (2005) 055019 [arXiv:hep-ph/0507113].

[49] E. Ma and G. G. Wong, Mod. Phys. Lett. A 3 (1988) 1561; H. Baer, X. Tata and J. Woodside, Phys. Rev. D 42 (1990) 1568.

[50] S. P. Martin and M. T. Vaughn, Phys. Lett. B 318 (1993) 331 [arXiv:hep-ph/9308222]. 